\title{
AN EFFICIENT PRIMER SELECTION PROCESS COMBINING \\ PROGRESSIVE AND ITERATIVE MULTIPLE SEQUENCE ALIGNMENT STRATEGIES: CLUSTALW AND HMMER
}

\author{
A Thesis \\ Presented to the Faculty of \\ California Polytechnic State University \\ San Luis Obispo \\ In Partial Fulfillment of the Requirements for the Degree \\ Master of Science in Computer Science
}

By

Michael C. Green

2011 
(C)2011

Michael C. Green

ALL RIGHTS RESERVED 


\title{
COMMITTEE MEMBERSHIP
}

TITLE:

AN EFFICIENT PRIMER SELECTION PROCESS COMBINING PROGRESSIVE AND ITERATIVE MULTIPLE SEQUENCE ALIGNMENT STRATEGIES: CLUSTALW AND HMMER

\author{
AUTHOR: MICHAEL C. GREEN \\ DATE SUBMITTED: JUNE 6, 2011
}

\author{
COMMITTEE CHAIR: Dr. Timothy J. Kearns \\ COMMITTEE MEMBER: Dr. Alexander M. Dekhtyar \\ COMMITTEE MEMBER: Dr. Anya L. Goodman
}




\title{
ABSTRACT

\begin{abstract}
AN EFFICIENT PRIMER SELECTION PROCESS COMBINING PROGRESSIVE AND ITERATIVE MULTIPLE SEQUENCE
\end{abstract} ALIGNMENT STRATEGIES: CLUSTALW AND HMMER
}

\author{
Michael C. Green
}

This thesis describes a method for using a computationally efficient algorithm to identify candidate DNA primer sequences. DNA sequencing primers are a critical element of polymerase chain reaction (PCR) and DNA sequence analysis. A variety of methods for deriving DNA primers exist, but such methods are often computationally intensive, or do not use available sequence data that could potentially serve as a possible resource for primer identification. Though no current algorithm exists which will always yield a correct primer for every need, evaluation of multi-sequence alignments may provide a reliable source for primer candidates. However, an exact mathematical solution for multi-sequence alignments, using currently available computational resources, is only viable for a very small number of sequences. Any solution for a larger number of sequences will therefore use other computational methods and heuristics to estimate an alignment.

The solution presented here, featuring a combination of ClustalW and HMMER alignment tools, is able to identify conserved regions in sequence data in a computationally efficient manner, and from these regions, suggest viable primer candidates. Computational complexity for the HMMER alignment effort has been maintained at $\mathrm{O}(\mathrm{MN})$; the suggested process for creating sequence alignments lead to a 15 -fold improvement in performance over conventional methods, while also successfully identifying fungal specific primers, with individual examples showing $90 \%$ or greater match for the given fungal phylum.

It was found that alignment quality could be further improved by using simple sorting methods against input sequence data. 


\section{ACKNOWLEDGMENTS}

First, without the tremendous patience, knowledge and guidance of my advisor, Dr. Timothy J. Kearns, this work would not exist.

I would also like to thank my committee members, Dr. Anya Goodman, and Dr. Alexander Dekhtyar for their warmth and insight, which they gave in equal measure.

My family and friends have graciously extended their constant support and considerable wisdom throughout this process. They include Aaron Jenrette, Sam Lund, Loreen Svobida, Don and Valerie Voita, Linda Sandy, Dara Manker, Amy Stets and Beth Cordova. None let me rest in doubt. My gratitude extends to each, as well as to others not named here.

Finally, the long days and nights at the keyboard would have been unbearable were it not for the unconditional companionship of Thom. I'm learning from him still. 


\section{Contents}

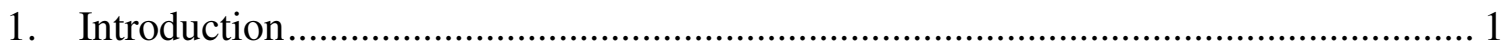

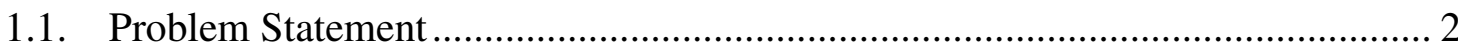

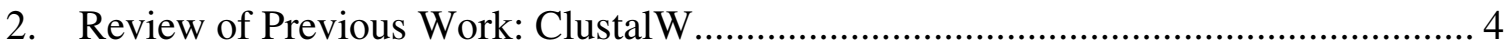

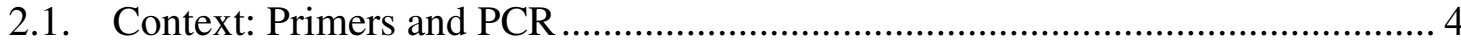

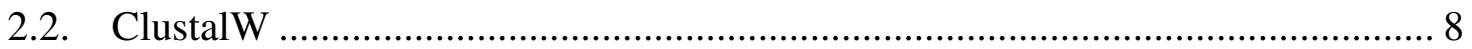

2.3. Computational Complexity of ClustalW ....................................................... 11

2.4. Example of Traditional Primer Finding Process Using Clustal........................... 12

3. Primer Identification Using Hidden Markov Models and HMMER ......................... 14

3.1. Hidden Markov Models ................................................................................ 14

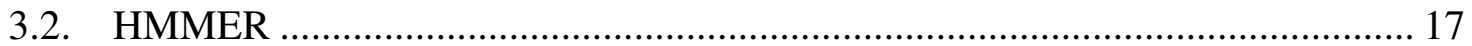

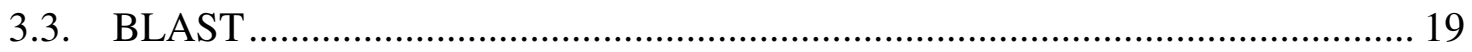

3.4. Solution Architecture of Primer Identification Using HMMER.......................... 21

3.5. Data for Testing HMM approach..................................................................... 24

3.5.1. Use of existing primers to group sequences ............................................... 25

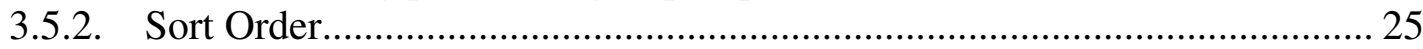

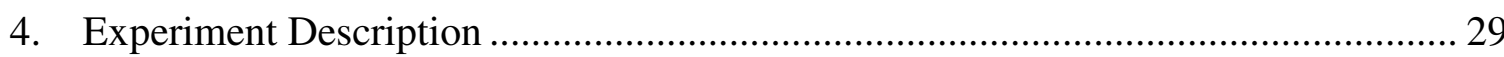

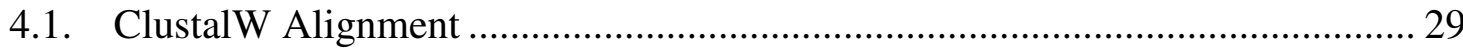

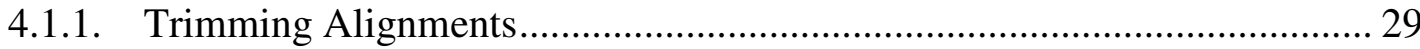

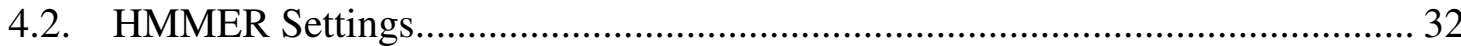

4.2.1. HMMER alignment and consensus ......................................................... 32

4.3. Consensus Analysis and Identification of Primer Candidates ............................. 33

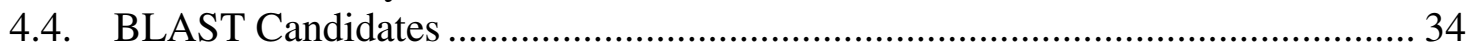

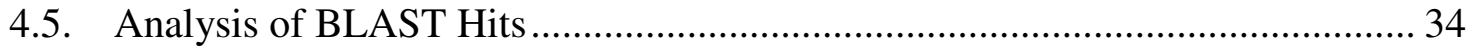

4.6. Test System Profile ..................................................................................... 34

4.7. Process for executing experiment using software interface................................ 35

4.8. Process for executing experiment- Detailed View............................................ 35

5. Experimental Results for HMMER Based Approach ................................................... 37

5.1. Timings for Individual Portions of the Experiment ........................................... 37

5.2. Number of Primer Candidates Generated ......................................................... 40

5.3. HMMER parameters and effect on alignment ................................................ 40

5.4. Effect of using unsorted sequence data on alignment success............................ 41

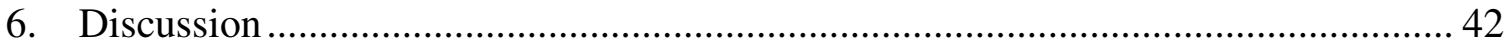

6.1. Timings for ClustalW and HMMER alignment processing .............................. 42

6.2. Organism Specificity of Primers Chosen............................................................ 43

6.3. Software Interface as an Improvement to Process ............................................ 51

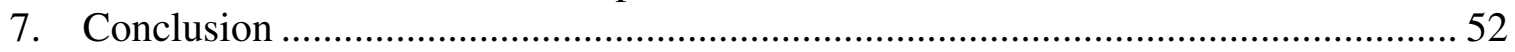

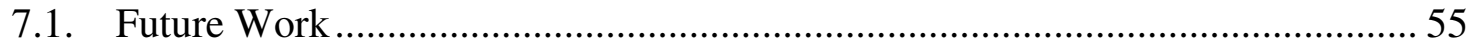

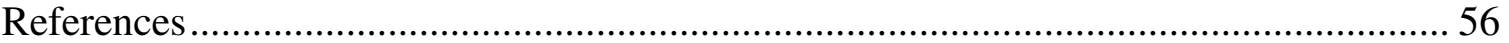

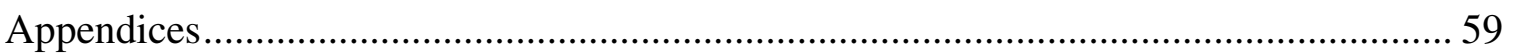

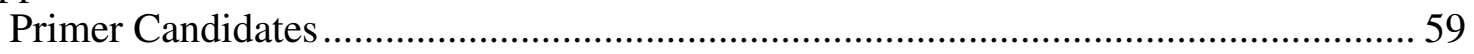

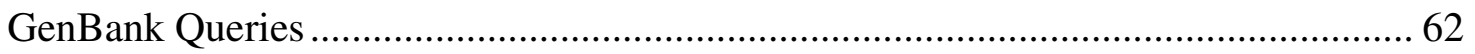

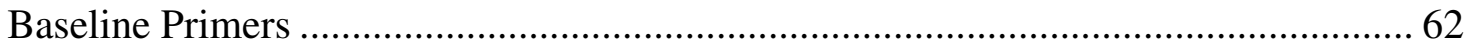

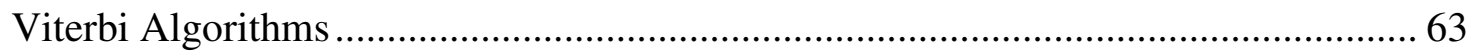

Process for executing experiment - Detailed View of Software Use............................. 65 
Description of Software Harness and Internal Scripts ......................................... 79

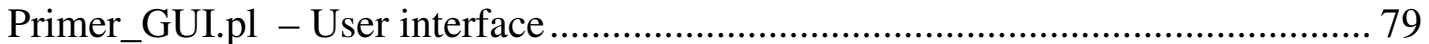

Generate_Primer_Candidates.pl - Alignment of Consesnus .................................... 79

Analyze_Netblast_Results.pl - Netblast............................................................ 79 


\section{Table of Figures}

Figure 1 - An example of a multiple sequence alignment created in Clustal ................... 3

Figure 2 - Primers enclosing a target region for amplification (top) and an ideal primer target region relationship (bottom) (Kampe, Kieninger\& Mecklenburg).......................... 6

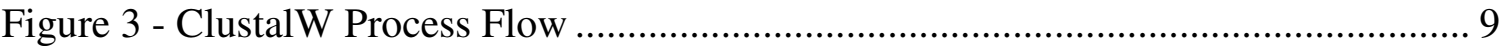

Figure 4 - Example of Traditional Approach to Primer Discovery Using Clustal ........... 13

Figure 5 An example hidden Markov model ........................................................... 16

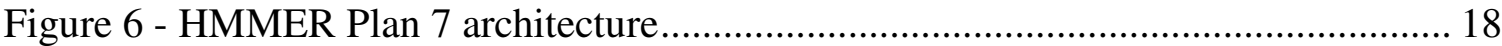

Figure 7 - Solution Architecture of primer identification using HMMER, Clustal and

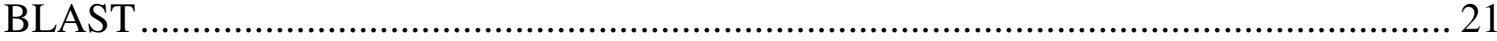

Figure 8 - Example of alignment editing, as viewed in ClustalX ................................. 31

Figure 9 - Software interface - Step 1 "Create BLAST Database” ............................... 66

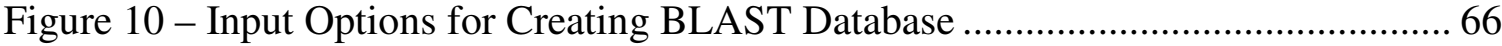

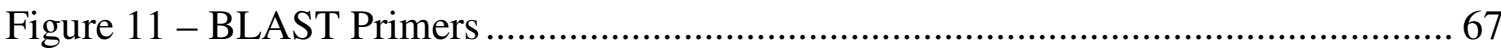

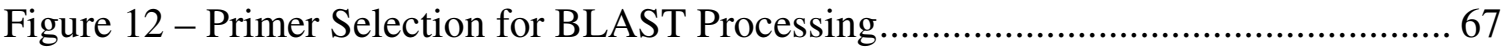

Figure 13 - Retrieve Matching Fasta Formatted Sequences ........................................ 68

Figure 14 - Select BLAST DB and Fasta Sequences for Sorting ................................. 68

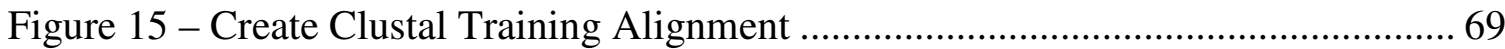

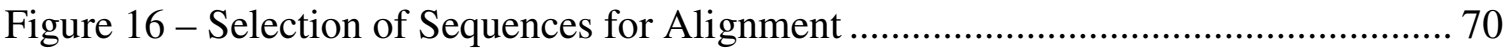

Figure 17- Generate HMMER profile......................................................................... 71

Figure 18 - Selection of ClustalW Alignment as basis for HMMER profile.................. 71

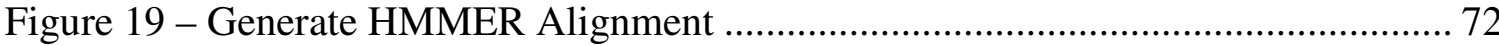

Figure 20 - Selection of Additional Sequences and HMMER profile for HMMER Alignment 
Figure 21 - Generate Primer Candidates …………….................................................. 74

Figure 22 - Selection of Primer Alignment for Primer Candidate Generation ................. 74

Figure 23 - NetBLAST Select Primers against GenBank ............................................ 75

Figure 24 - Primer Selection for GenBank BLAST Processing......................................... 76

Figure 25 - Evaluate NetBLAST Results ................................................................ 77

Figure 26 - Select NetBLAST Results for Evaluation .................................................. 77 


\section{List of Tables}

Table 1 - Naming Convention for Data and Primer Labels ......................................... 27

Table 2 - Summary of Sequence Data Statistics for Each Dataset ............................... 28

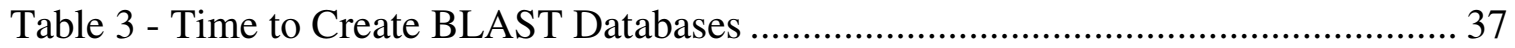

Table 4 - Time to Complete ClustalW Alignments ................................................... 38

Table 5 - Time to Create HMMER Profile Based on Initial ClustalW Alignment........... 38

Table 6 - Time to Complete HMMER Alignment.................................................... 39

Table 7 - ClustalW baseline alignment timings ...................................................... 39

Table 8 - Summary of BLAST Hits for Primer Candidates with Fungal Specificity. Upper case characters represent ClustalW aligned data; lower case characters represent nucleotides aligned only in HMMER.

Table 9 - Summary of BLAST Hits for Primer Candidates with Specificity for Ascomycota. Upper case characters represent ClustalW aligned data; lower case characters represent nucleotides aligned only in HMMER.

Table 10 - Summary of BLAST Hits for Primer Candidates with Specificity for Basidiomycota. Upper case characters represent ClustalW aligned data; lower case characters represent nucleotides aligned only in HMMER.

Table 11 - List of Primer Candidates Evaluated and Primer Candidate Characteristics. Upper case characters represent ClustalW aligned data; lower case characters represent nucleotides aligned only in HMMER. 


\section{Introduction}

This thesis describes a novel computational technique to identify DNA primer sequences, which have important applications in molecular biology. Current techniques are limited to small data sets or require long computational runtimes. The problem under consideration is how to efficiently identify primers, short DNA sequences used to enable the rapid amplification of a targeted DNA sequence, within a large multi-sequence alignment. Primer identification must be done frequently, since primers are typically specific for each particular problem and may need to be organism-specific, or reflect other necessary constraints. The multi-sequence alignment tool, ClustalW, can be used in identifying potential primer regions, but requires several hours runtime when attempting to align several hundred protein sequences (Oliver et al.). This project aims to develop a more efficient primer identification process.

The work described in this thesis was initiated as an effort to aid a group of biologists in identifying fungal species existent in soil samples. As Polymerase Chain Reaction (PCR) would be used to amplify sample data, the process could benefit from an improved means of primer development. This could best be accomplished by identifying primers that are as universal as possible for fungus hybridization while not hybridizing other non-fungal eukaryotes. Primers that can aid in identification of individual fungal families or species would be ideal. Software and automated processes that can assist primer design would therefore prove valuable and timesaving. Improved tools would allow researchers to focus efforts on results generated from PCR, rather than developing primers for use in PCR. 


\subsection{Problem Statement}

Given a large quantity of sequence data, 500 sequences or more, is it possible to develop software that efficiently identifies valid primers for a specific use, based on conserved regions within the existing sequence data?

Identifying primers generally requires computing alignments for a large number of sequences, and applying methods that identify conserved regions - areas of highly similar sequence data that may imply similar function for between related organisms. Conserved regions are potential target sites for primers, therefore, likely sources for developing new primer candidates.

Once an alignment has been created it would be possible to identify conserved regions that could yield new primer candidates. The problem then is to develop a method of aligning large numbers of sequences in such a way that conserved regions can be used for primer identification, while processing the sequences in an efficient manner.

A multiple sequence alignment in this context is when two or more DNA sequences have been lined up to match as many identical residues or conservatively substituted positions as possible (Baxevanis, Ouellette). It is often represented as a matrix of characters, where each organism's string reads horizontally, and common characters and substrings, relative to the position in the sequence string, are aligned vertically. To illustrate, an example of a multiple sequence alignment that has been created using ClustalW is shown in Figure 1 below.

The computational complexity involved in creating an optimal multiple sequence alignment is significant. That is, as the number of sequences grows the computational requirements of all current approaches, for an exact solution, increases very rapidly; the computational requirements expand in proportion to the size of the data set, exceeding the range of existing or possible 
computing resources. Widely accepted alignment software, such as the variants of Clustal, are limited by memory and runtime constraints for moderate sized problems; experiments show runtime can double for each additional increment of 200 sequences aligned with ClustalW (Oliver et al.).

This work focuses on hidden Markov models, and software based on them, which can be used to build large sequence alignments, offer reduced runtimes, and support the identification of conserved regions likely to yield primer candidates.

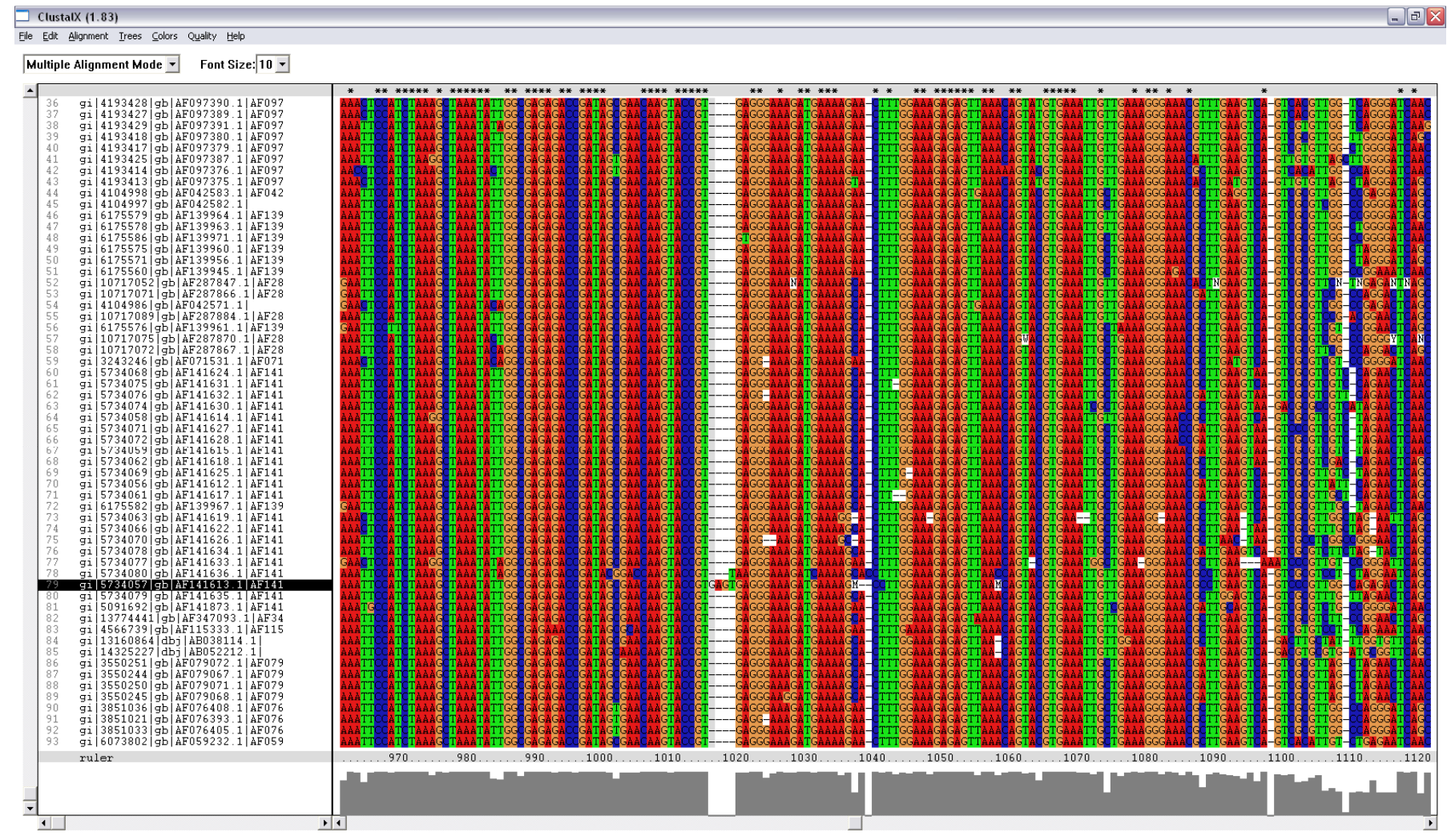

File d:/perl_primer/clustal/alignments/its4_basidio_fa_1-100_ed.aln sa

Figure 1 - An example of a multiple sequence alignment created in Clustal 


\section{Review of Previous Work: ClustalW}

This section describes primers and their use in Polymerase Chain Reaction (PCR), and the process of PCR is outlined. Basic constraints intended to ensure primers viability are discussed. A review of Clustal and its variants follows, with an overview of the Clustal alignment algorithm, noting its computational complexity.

\subsection{Context: Primers and PCR}

"The design of appropriate PCR sense and antisense...is not yet part of any standardized algorithm, and several sets of rules have been proposed to govern primer sequence selection."

(Lowe et al.)

Deoxyribonucleic acid (DNA) molecules are double-stranded polymers consisting of the nucleotide bases adenine (A), cytosine $(\mathrm{C})$, guanine $(\mathrm{G})$ and thymine $(\mathrm{T})$. Each base has a specific complement to which it binds - adenine binds to thymine, and cytosine binds to guanine - forming a base pair. Each strand of DNA has a specific orientation, and the double strand has a complementary relationship. DNA contains the genetic code of the organism, as the sequence of nucleotide bases encode the amino acids, and in turn, the proteins that define organism. Replication of DNA is essential to each organism, and allows passage of hereditary information to descendants.

PCR is a technique that mimics DNA replication for target regions of DNA, and aids the study of gene function, phylogenic relationships between organisms and hereditary disease. PCR also has numerous applications in medicine, forensics, and research related to infectious disease. 
DNA primers, when used in PCR, are considered in primer pairs, known as the forward and reverse primers, with each primer pair bracketing a sequence area of interest. The primers enable hybridization of complementary DNA strands towards one another during PCR replication, and are critical to the amplification process for a specific region of DNA.

PCR operations consist of three steps. First, a high temperature phase (95 C) denatures double stranded DNA sequences into single strand DNA sequences. In the second phase, under low temperatures (45-65 C), the process of hybridization or annealing begins, where primers are used as initializing templates for the eventual DNA sequences. Primer extension, where the sequence is duplicated, occurs in the third phase, at an intermediate temperature $(72 \mathrm{C})$. These three phases are repeated 25-45 times, resulting in millions of copies of the target sequence (Kampke, Kieninger, and Mecklenburg).

As with the DNA strands that are the subject of replication, primers are sequences of the nucleobases adenine, cytosine, guanine, and thymine, represented by the characters A, C, G, and T respectively. 

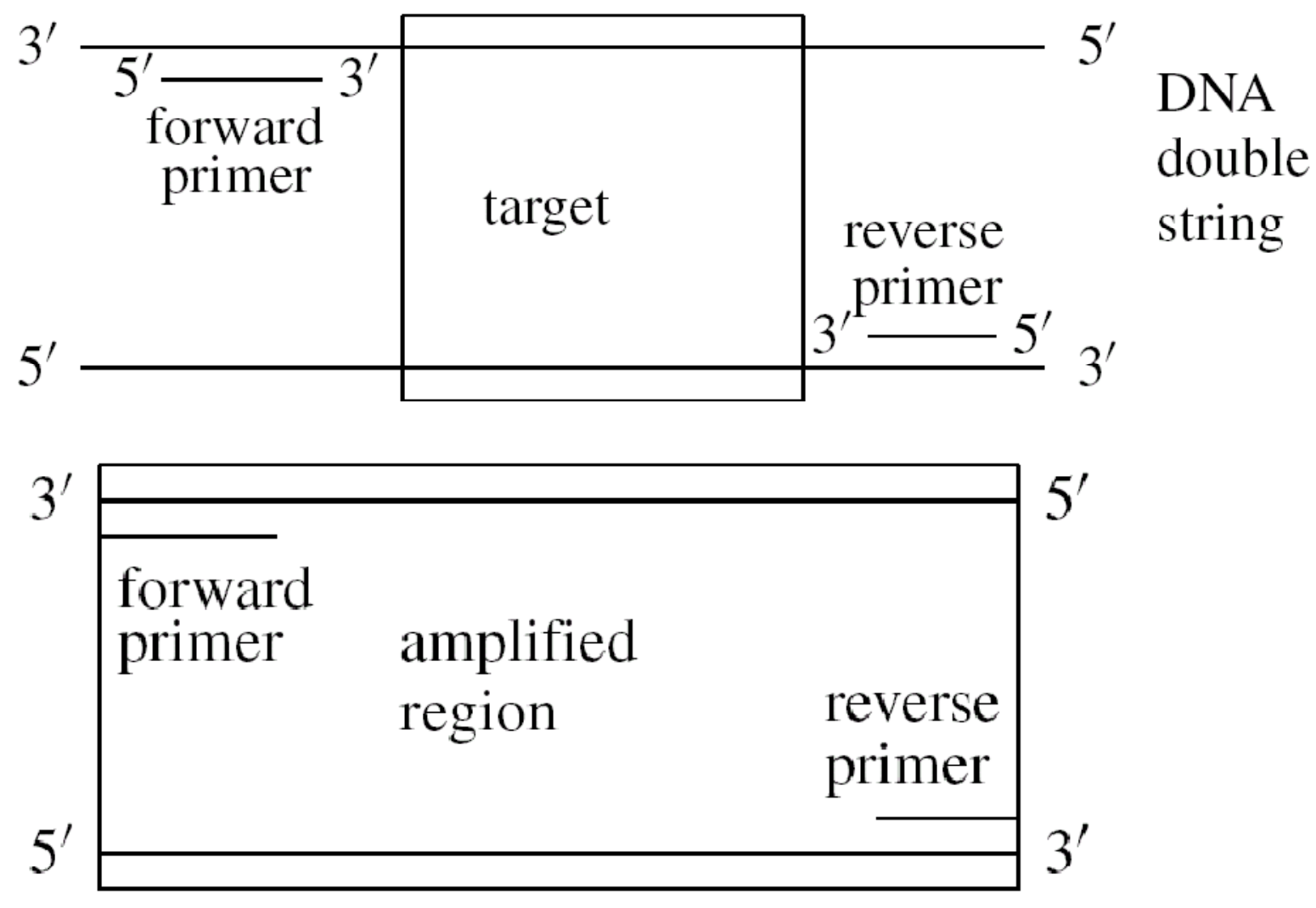

Figure 2 - Primers enclosing a target region for amplification (top) and an ideal primer-target region relationship (bottom) (Kampe, Kieninger\& Mecklenburg).

The nucleotide in the first primer position of the primer string sequence, relative to the direction it will be replicating, is known as the 5' ("five prime") end, the last is known as the 3 ' ("3 prime") end, as shown in Figure 2. Qualities that can be used to select primers include primer length, the proportion of guanine and cytosine or GC content, melting temperature and potential for selfhybridization. Primer pairs are evaluated for the predicted success in the specified PCR operation, as no specific primer pair is consistently more effective than all others (Kampke, Kieninger, and Mecklenburg).

Selection constraints can be used to identify and isolate potentially useful primers, and eliminate those that are not as likely to be suitable for use in PCR. Primer length is typically 18 to 24 base 
pairs. The GC content, the proportion of nucleotides $\mathrm{G}$ and/or $\mathrm{C}$, usually represented as a percentage, should be between $45 \%$ and $55 \%$; the GC content is important in determining the annealing temperature of the primer. Both members of a given primer pair should anneal within a close temperature range, otherwise the PCR process will likely fail during the hybridization phase for one or both primers.

It is desirable for the 3' end of a primer to contain some combination of a GC pair, such as CC, GG, GC or CG, as this may aid initiation of complementary strand formation. This is known as the GC clamp, and may also be satisfied by no more than 3 single $\mathrm{G}$ or $\mathrm{C}$ nucleotides in the last few bases near the 3' end of the primer (Burpo) (Lowe et al.).

Primers should be avoided if they are likely to self-hybridize or hybridize to the complement of the pair primer. Limiting the number of contiguous nucleotides that match any other portion of the same primer or the primer pair complement can control this (Lowe et al.).

A key component of this thesis is the use of queried sequence datasets to find multiple primer candidates relevant to the given dataset. The experiment described below begins by establishing known regions of conserved rDNA sequences, which may contain regions and sub-regions that are conserved and specific to organisms of interest. Creating alignments of sufficient size and complexity to reveal these regions requires larger alignments than is typically practical using standard clustering techniques such as ClustalW-based sequence alignment software alone. 


\subsection{ClustalW}

In this section, ClustalW and the Clustal algorithm are briefly outlined, and the computational complexity of ClustalW is presented. ClustalW and its variants have long been standard tools for use in multiple sequence alignment tasks, using a progressive approach to multiple sequence alignment (Chenna et al.) (Notredame).

The ClustalW implementation offers performance and feature improvements over previous versions. ClustalX adds a graphical user interface, suitable for creating and editing alignments. Visual review of a resulting alignment can aid alignment assessment and further editing of alignment may be necessary. ClustalX provided the visual review and editing capability necessary for the initial sequence alignment in this experiment.

The Clustal sequence alignment algorithm consists of three steps, as highlighted in Figure 3 below:

1. A distance matrix is derived from a pair-wise comparison of all sequences

2. A guide tree is created, based on the distance matrix.

3. The sequences are aligned in a progressive manner. The guide tree determines the order in which sequences are aligned.

(Thompson, Higgins, and Gibson) 


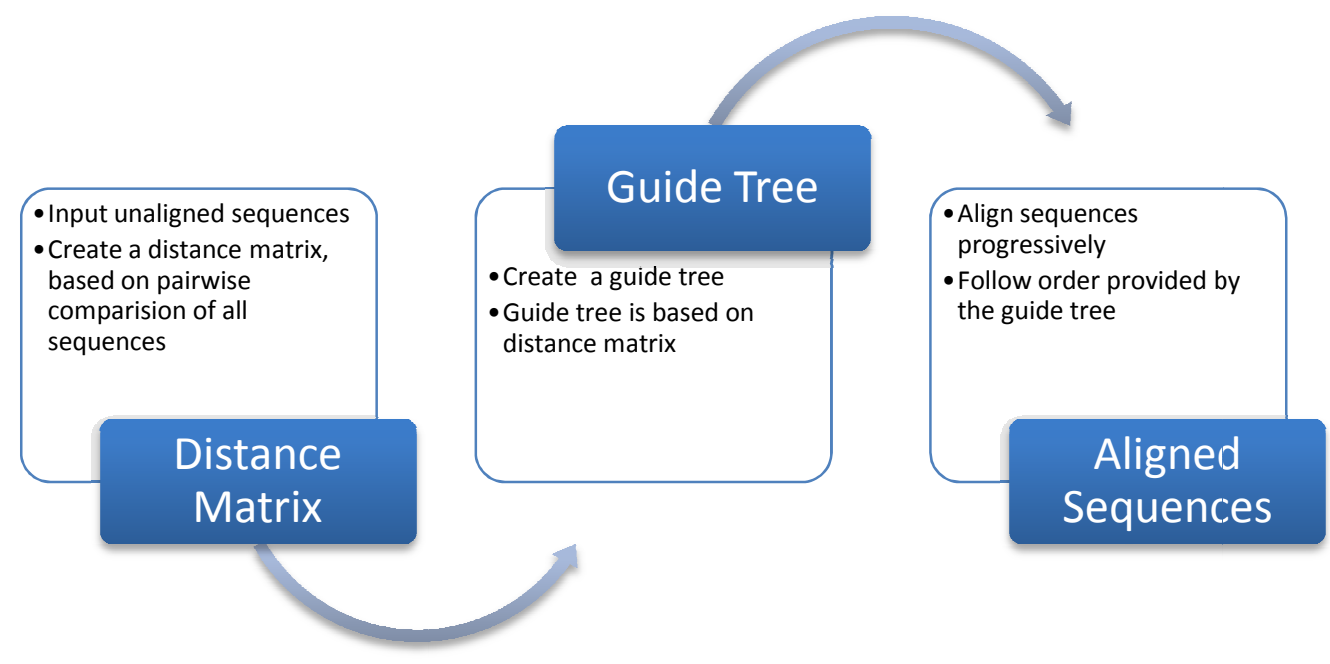

Figure 3 - ClustalW Process Flow

Each sequence pairing receives a score during the alignment process; the score is a measurement of relative alignment success. The scoring process takes into account penalties for gap creation and gap extensions, and reflects the ratio of matched nucleotides to the number of nucleotides compared. The resulting distance matrix produced in the first step is the basis of the guide tree, which will set the order of the sequence for the final, progressive alignment. ClustalW, based on the earlier version ClustalV, introduces a choice between faster distance matrix estimation, or a slower method with improved accuracy, using dynamic programming.

Creation of the unrooted guide tree is completed using the Neighbor-Joining method, and is based on the earlier distance matrix. The mid-point is established as the root, with equal length branching on either side. The branch length represents the expected relative divergence of the sequences. The guide tree relies on the premise that sequence similarity implies evolutionary proximity of the sequence organisms, and models the phylogenetic relationship of the sequences (Thompson, Higgins, and Gibson). 
Dynamic programming is used during the final progressive alignment to perform pair wise sequence comparisons, with the guide tree providing the order of the pairings. Pairings can be between sequences or previously completed alignments. The process of alignment includes the use of a weight matrix for residues, and calculation of gap penalties for introduction of new gaps, or extension of existing gaps. The selection of sequences for alignment begins at the tips of the guide tree, where sequences estimated to be most closely related are clustered together. The process of building the alignment progressively works towards the center or root of the guide tree (Thompson, Higgins, and Gibson).

According to Thompson, et. al., the final progressive alignment is most computationally intensive during the alignment of two sequences, or during the alignment of two groups of sequences. This process employs the Myers-Miller dynamic programming algorithm, noted by the authors for its efficiency (Thompson, Higgins, and Gibson).

The authors suggest it is reasonable to more finely edit the resulting alignment manually, based on visual cues in its structure. For example, this might include the removal of sequence outliers that have introduced numerous insertions to otherwise conserved sequence regions. Alternatively, it is possible to select sequences or groups of sequences that can be re-aligned separately, and then re-introduced to the larger alignment. ClustalW's ability to determine a best alignment is summarized as follows: "Nonetheless, if an alignment is impossible to improve by eye, then the program has achieved a very useful result.” (Thompson, Higgins, and Gibson)

Manual refinement is expected with divergent sequence alignments. Further instructions and heuristics for this type of alignment management are included in the original article (Thompson, Higgins, and Gibson). Manual editing is most often necessary to remove unrelated or dissimilar sequences, which have caused the introduction of excessive gaps. With ClustalW, gaps are 
introduced early in the alignment process; gap placement is not changed as more sequences are added to the alignment. The fact that the gap placement is not changed is generally not an issue, since the first sequences to be aligned are expected to be more closely related. Gaps introduced at this point are more likely to be accurate than those introduced for more distantly related sequences. It is possible for sequences to introduce gaps or to create a less than optimal alignment, and obscure otherwise conserved regions. ClustalW improvements over prior versions have been shown to create successful alignments, using only the default parameters associated wth gap introduction and gap extension penalties. Even within sequences datasets that are no more than $35 \%$ identical, blocks of similarity will be apparent, and provide a reasonable alignment that can be further refined through editing. Sequences that are divergent can be removed entirely, or removed and added again to the alignment (Thompson, Higgins, and Gibson).

The solution suggested in this thesis will not eliminate the possibility for such refinement, but the process has been limited to only a smaller seed alignment, and only eliminated the most obvious examples of divergence within the sequence group. An example would be a sequence that introduces a several hundred base gap to either the beginning or end of the alignment, to which no other sequences have aligned. Further work may show that this type of editing is not necessary in the proposed solution.

\subsection{Computational Complexity of ClustalW}

The computational complexity of ClustalW has been assessed and described in a 2004 article (Edgar). 
The complexity can be summarized as follows. The computation of the distance matrix in step 1 , the initial pair wise comparison, for average sequence length $\mathrm{L}$, with $\mathrm{N}$ representing the number of sequences, is $\mathrm{O}\left(\mathrm{N}^{2} \mathrm{~L}^{2}\right)$ in time complexity and in $\mathrm{O}\left(\mathrm{N}^{2}+\mathrm{L}\right)$ space complexity. This is based on the use of the Myers-Miller linear space algorithm, which, given average sequence length L, is $\mathrm{O}(\mathrm{L})$ in space and $\mathrm{O}\left(\mathrm{L}^{2}\right)$ in time. The computational complexity is driven by the number of sequence pairings, represented by $\mathrm{N}(\mathrm{N}-1) / 2=\mathrm{O}\left(\mathrm{N}^{2}\right)$ pairings.

The neighbor-joining method for creation of the guide tree in step two of the ClustalW algorithm is stated as $\mathrm{O}\left(\mathrm{N}^{2}\right)$ space and $\mathrm{O}\left(\mathrm{N}^{4}\right)$ time, though possibly $\mathrm{O}\left(\mathrm{N}^{3}\right)$, depending in part on the version of ClustalW used. ClustalW 1.82 was cited in this example.

The final progressive alignment is expressed as $\mathrm{O}\left(\mathrm{NL}+\mathrm{L}^{2}\right)$ in space, and $\mathrm{O}\left(\mathrm{N}^{3}+\mathrm{NL}^{2}\right)$ in time, with cumulative computational complexity for ClustalW summarized as $\mathrm{O}\left(\mathrm{N}^{2}+\mathrm{L}^{2}\right)$ in space, and $\mathrm{O}\left(\mathrm{N}^{4}+\mathrm{L}^{2}\right)$ in time (Edgar).

Clustal has been further developed for use on multi-core systems. While performance speeds increased in these implementations as much as 10 times, it required systems with 16 CPUs, which may not be available for common lab use (Chenna et al.).

\subsection{Example of Traditional Primer Finding Process Using Clustal}

In Figure 4, an example of how Clustal currently may be used to identify primers is shown. Either ClustalW or ClustalX could be used, or both. ClustalW is typically executed via a command line interface, which lends itself to scripting and batch processing. ClustalX is accessed via a graphical user interface; alignments can be viewed in an interactive mode, which can include color-coding for nucleotide bases. Alignments created in ClustalW can be opened and viewed in ClustalX. Primer identification would rely on the observations of the researcher, 
as the process does not produce primer candidates may be obser primer-by-primer basis, or requi process introduced and demonst simultaneously and proposed, un

Figure 4 - Example of Traditional AI 


\section{Primer Identification Using Hidden Markov Models and HMMER}

Hidden Markov models are briefly introduced in this section, with a description of HMMER, a sequence alignment toolset implementation based on hidden Markov models. The solution architecture will be described, in terms of sequence processing and how the alignment is created, and primer candidates are selected.

\subsection{Hidden Markov Models}

The premise of hidden Markov models, like that of other statistical models, is that sample data can be used draw general observations and conclusions about other similar data. This assumes the data available has the potential to lend itself to broader characterization regarding its nature and inherent qualities. Sequence data is well suited for hidden Markov models, the probabilistic formulization of linear sequence or time series information (Koski).

Hidden Markov models (HMMs) were introduced to computational biology by Churchill in 1989 (Churchill).

The hidden Markov methods can be viewed as stochastic regular grammars that generate sequences from a given alphabet. A HMM consists of states that emit characters: transition probabilities define the moves among the HMM states and the emission probabilities that describe the character distribution in a given state. The path through the states is Markovian (i.e. it only depends on the previous state) but unknown from the outside (therefore 'hidden'). If let to run free, a HMM produces data according to the probabilistic model. (Loytynoja and Milinkovitch) 
A general and a formal definition for hidden Markov model follow.

Definition: A Hidden Markov model is a variant of a finite state machine having a set of states, Q, an output alphabet, $\mathrm{O}$, transition probabilities, A, output probabilities, B, and initial state probabilities, $\Pi$. The current state is not observable. Instead, each state produces an output with a certain probability (B). Usually the states, Q, and outputs, O, are understood, so an HMM is said to be a triple, $(\mathrm{A}, \mathrm{B}, \Pi)$.

Formal Definition:

- $A=\left\{a_{i j}=P\left(q_{j}\right.\right.$ at $t+1 \mid q_{i}$ at $\left.\left.t\right)\right\}$, where $P(a \mid b)$ is the conditional probability of $a$ given $b, t \geq 1$ is time, and $q_{i} \square Q$. $\square$ Informally, $A$ is the probability that the next state is $q_{j}$ given that the current state is $q_{i}$.

- $B=\left\{b_{i k}=P\left(o_{k} \mid q_{i}\right)\right\}$, where $o_{k} \square O$. $\square$ Informally, $B$ is the probability that the output is $o_{k}$ given that the current state is $q_{i}$.

$\Pi=\left\{p_{i}=P\left(q_{i}\right.\right.$ at $\left.\left.t=1\right)\right\}$ (Black).

In principle, a hidden Markov model for use in sequence alignment would consist of three types of states; match states, insertion states and deletion states. Each state would include the probability for transitions to each of the other states, or to itself, for a total of nine possible transition types. Examples would include match state to insertion state, insertion state to itself or delete state to match state. A match states include correspondence probabilities for each of A, T, $\mathrm{C}$ and $\mathrm{G}$; there are not independent match states for each of the possible symbols.

Each hidden Markov model, similar to a finite state automata, or FSA, represents a series of interconnected states. Each state emits an output symbol, which in the case of a nucleotide sequence, is a single nucleotide. Two parameters are associated with each state. The first 
parameter is the state emission probability, representing the probability of emitting a particular symbol from the set of possible symbols. The second parameter is the state transition probability, representing the probability of changing to a new state from the current state. The complete sequence is formed by starting from an initial state and moving between states until arriving at the terminal state.

A toy model (Eddy, 1998) in Figure 5 illustrates the relationship of states with probabilities, transitions and hidden versus output symbols in a hidden Markov model.
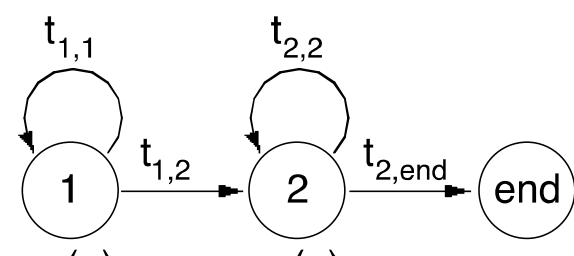

HMM

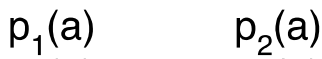

$p_{1}(b)$

$\mathrm{p}_{2}(\mathrm{~b})$

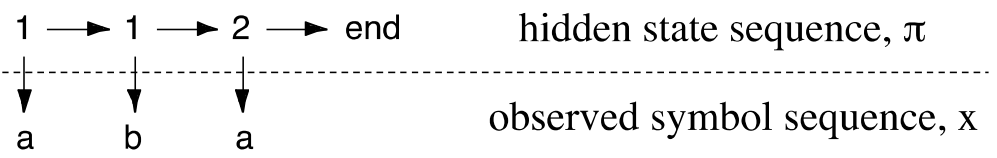

$$
t_{1,1} t_{1,2} t_{2, \text { end }} p_{1}(a) p_{1}(b) p_{2}(a)=P(x, \pi \mid H M M)
$$

\section{Figure 5 An example hidden Markov model}

The sequence of states described is a first-order Markov chain. First order Markov chains are not directly observable; only the final output symbols in the sequence are observable, hence the term "hidden Markov model." An HMM profile, which contains position-specific probabilities for a given alignment of sequences, can be generated using a training group of unaligned sequences, or use as input a previously aligned sequence set, and then serve as a guide for a much larger multi-sequence alignment (Eddy, 1997). In this experiment, the larger alignment was guided by a HMMER profile, which had been trained using of a small ClustalW alignment. Sequences for 
the ClustalW training alignment, and the final complete alignment come from the same dataset in each variation of the experiment run. Each sequence dataset has been sorted by sequence length. The first 100 sequences by sort order were used in the training alignment; sequences that followed, in order by length, were used for the large alignment. This approach combines initial findings by Thompson, which concluded that sequence length can impact alignment quality, and suggestions by Eddy that using pre-aligned sequence data can result in higher quality alignments than using unaligned data alone (Thompson, Plewniak, Poch) (Thompson, Higgins, and Gibson) (Thompson, Higgins, \& Gibson) (Eddy, 1998).

Within a given alignment of sequences, a state is corresponds to a consensus column position. It is possible to have insertion and deletions, allowing for the insertion of symbols, or skipping of positions.

\subsection{HMMER}

HMMER, developed by Sean Eddy, is an HMM-based implementation of a series of related software tools for use in sequence analysis. It includes not only multiple sequence alignment processing, but HMM profile database management tools and other functionality. 


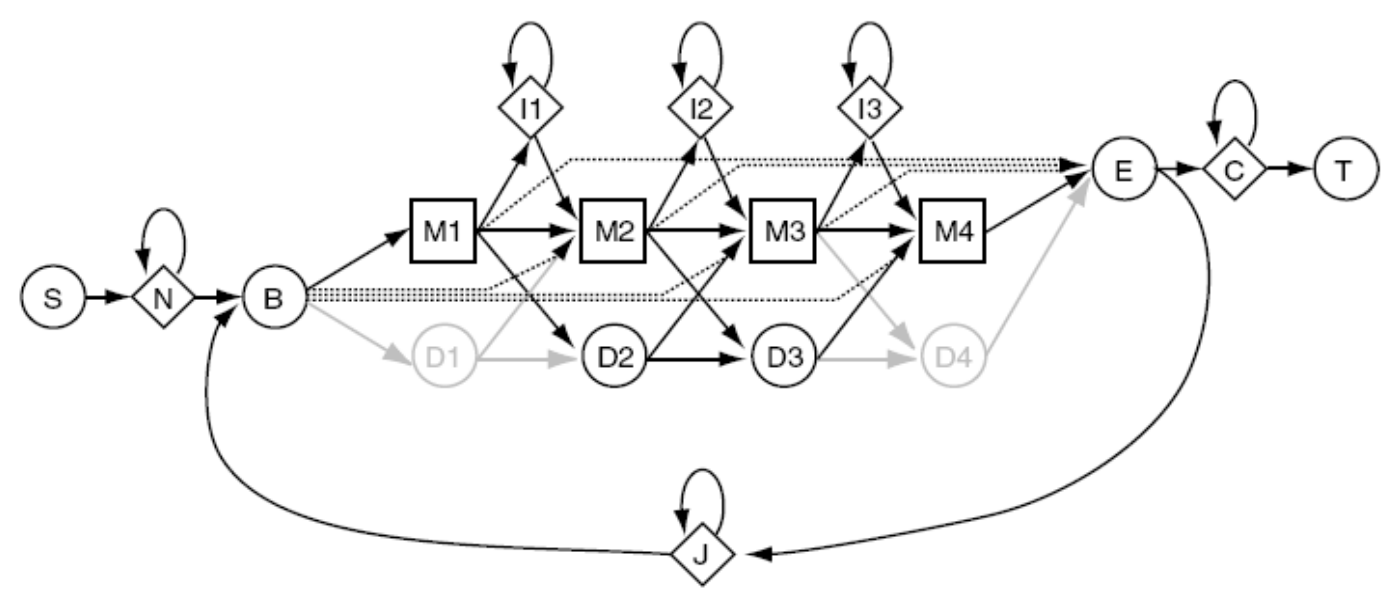

Figure 6 - HMMER Plan 7 architecture

"The Plan7 architecture. Squares indicate match states (modeling consensus positions in the alignment). Diamonds indicate insert states (modeling insertions relative to consensus) and special random sequence emitting states. Circles indicate delete states (modeling deletions relative to consensus) and special begin/end states. Arrows indicate state transitions.” (Eddy, 2003)

Figure 6 illustrates the core of the HMMER implementation, the Plan 7 architecture, which uses seven - hence the name - possible transition paths, omitting insert-to-delete state and delete-toinsert state paths that would ordinarily be available in a HMM, as previously mentioned. The key artifact produced using HMMER during the training process is an HMM profile, which is unique to the group of sequences which are being assessed. The HMM profile contains all the probabilities for emissions and transitions inherent to its corresponding dataset. 
Though generally more accurate, ClustalW sequence alignments are limited by the number of sequences that can be aligned at one time. HMMER has the potential to create much larger successful alignments; some containing tens of thousands of sequences

(Eddy, 1995) (Eddy, 1997).

HMMER computational complexity is summarized by the software author as having a worstcase algorithmic complexity of $\mathrm{O}\left(\mathrm{NM}^{2}\right)$ in time and $\mathrm{O}(\mathrm{NM})$ in space, where sequence length is represented by $\mathrm{N}$, and $\mathrm{M}$ reflects the number of states in the hidden Markov model. HMMER computational complexity improves to $\mathrm{O}(\mathrm{MN})$ when using profile hidden Markov models with a constant number of state transitions per state. Two algorithms serve as the basis of the HMMER implementation: The Viterbi algorithm, variations of which are available in more detail in the appendix, provides the underlying alignment algorithm, while the Forward algorithm implements alignment probability scoring. Some computational overhead is avoided when using a prealigned sequence alignment to create the hidden Markov model, as the observed sequence states can easily be converted into probabilities (Eddy, 1998). Since this experiment will be using prealigned data to create HMM profiles for each dataset, expected computational complexity will be maintained at $\mathrm{O}(\mathrm{MN})$.

\subsection{BLAST}

Though not required in the solution architecture that will be described shortly, BLAST software adds highly useful additional functionality; BLAST can be a means of gathering potential sequence data for use in sequence analysis and primer identification, and BLAST can be used to partially validate primer candidates. 
BLAST, or "Basic Local Alignment Search Tools," introduced in 1990, is available at the National Center for Biotechnology Information, (http://www.ncbi.nlm.nih.gov/blast/). Typical use involves a sequence query string, which is compared against a database of sequences, returning "hits" or matches to the query string in a standardized format. The query string may be a protein or nucleic sequence, and can be submitted for query in a number of combinations of sequence and databases, such as protein sequence against protein database, nucleic sequence against nucleic database, but also protein sequence against nucleic database, or nucleic sequence against protein database.

Evaluation of the query string against sequences in the search space, for example, a database of sequences, is performed using a pair-wise method. Results are in the form of a similarity score, and the scores for matches are ranked. The search conducted is for a local alignment, meaning not all of the sequence is used in the alignment match, unlike a global alignment, as seen in ClustalW and HMMER. In many cases, the default parameters will be sufficient, but the algorithms used are designed for speed, not sensitivity. As such, some matches will be missed, and there is no guarantee that the search will find the best hit possible.

Typical searches can be executed through the NCBI web interface, but the BLAST executables are available for most computing platforms, and can be downloaded and used for creation of local, standalone databases. The solution design of this project makes use of both. A local sequence database was created for storing sample datasets, and NetBLAST, a scriptable interface for performing BLAST searches against NCBI databases, was used for validation of primer candidates.

The format for query results is the same for the hosted and standalone version of BLAST, with a report consisting of three components. The first section is a graphical representation of the hit, 
or match, with an ASCII character representation of the matched amino acids or nucleotides. The second portion lists a description of hits, their accession numbers, expect scores (or the GI number and score). In the web interface, the GI number can be selected, as a hyperlink, in the third portion of the report, to take the user directly to the GenBank entry for the match. The standalone version can be configured to create an HTML report with the same functionality, though the default is plain text (Misener and Krawetz).

\subsection{Solution Architecture of Primer Identification Using HMMER}

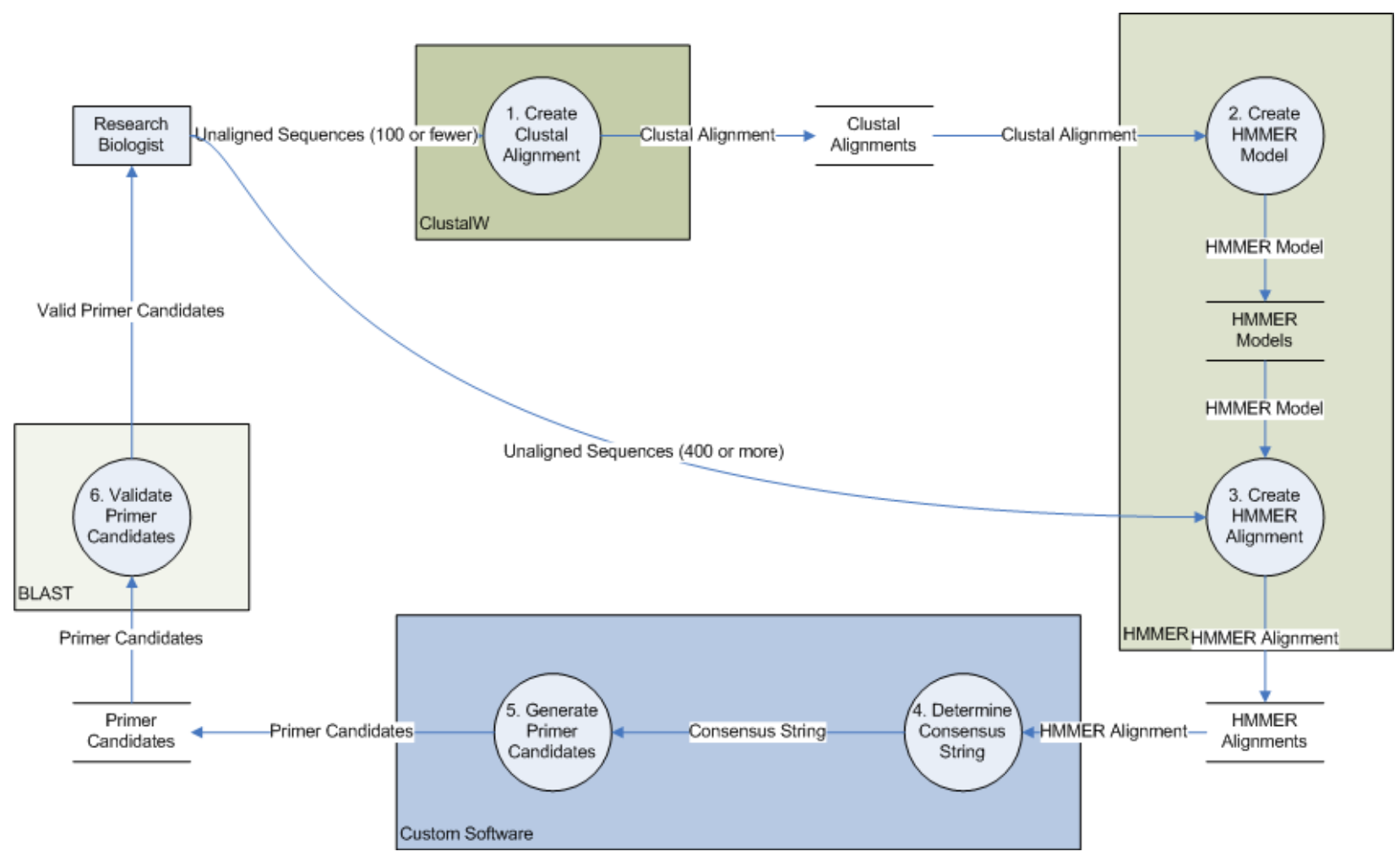

Figure 7 - Solution Architecture of primer identification using HMMER, Clustal and BLAST 
In the solution design proposed by this thesis (Figure 7 - Solution Architecture) a hybrid of new software and existing software has been integrated within a simple user interface. ClustalW, HMMER and BLAST, which have been described above, have been chosen because of their widespread use and acceptance for sequence alignment and manipulation, but more importantly, for their effectiveness when combined, in identifying conserved regions likely to contain primer candidates.

The approach explored in this project follows the steps listed below.

1. Create a ClustalW sequence alignment of 100 sequences or fewer.

2. Create a HMMER profile using the ClustalW alignment from step 1 as input.

3. Use the HMMER profile as a guide to create a final sequence alignment, adding up to 400 additional sequences as input to the HMMER software.

4. From the resulting multi-sequence alignment, derive a consensus, preserving conserved regions.

5. Evaluate the consensus and the conserved regions contained within, for primer candidates, applying necessary primer constraints. This includes calculating melting temperature and GC content percentage for each primer candidate.

6. Validate primer candidates with BLAST searches against the GenBank sequence database. While this step is not core to the primer generation, it has been included here as a fully proposed solution, which includes basic primer validation.

Use of ClustalW, which has been described earlier, allowed the creation of relatively small, (100 sequences or fewer) high quality training alignments, sufficient to build the HMMER profiles, 
which would in turn guide the later and larger HMMER alignments. Default parameters proved sufficient for most operations. Training the HMMER profiles in this way reduces the computational complexity for HMMER, and reduces potential training pitfalls, according to Eddy (Eddy, 1998).

HMMER-generated larger alignments (101 to 400 sequences) from which a consensus could be derived and evaluated for conserved regions of genetic data. HMMER toolset includes the tool "hmmemit," which can generate a probabilistic consensus, based on a particular HMM profile. The decision was made to use the HMMER alignment directly for consensus review, resulting in a consensus that provided a broader and more accurate reflection of conserved regions within the sequence data. HMMER parameters used were default or simple to chose (i.e. output file name), with the exception of gap parameters. Discussion will follow regarding a problem encountered during the alignment process, and the resulting choice of parameters from the default value for 'gapmax', which resolved the issue.

BLAST was used to validate primer candidates against the GenBank sequence database, in terms of the number of organisms returned and by the taxonomy matches observed. For most BLAST operations, default or simple parameter choices were sufficient.

Supporting scripts and the user interface were written in Perl and Perl/tk. Perl implementations can easily be made portable, with little to no modifications. Perl is widely used in sequence analysis, due in part, to Perl's strength in handling string matching and manipulation, and use of regular expressions, appropriate for sequence and consensus analysis. Perl/tk, which provides graphic interface support for Perl implementations, is not as effective a tool for user interface implementations, but the resulting code is transparent and relatively easy to adapt and modify. 
The remaining scripts and software for analysis, primer candidate proposal, evaluation, and primer candidate verification were written in Perl as well.

The proposed solution architecture relies upon the use of HMMER, ClustalW and BLAST. Each tool has been ported to a number of operating systems, including Windows, Mac OS X and various flavors of UNIX. Standard sequence and alignment formats have been used for the files, sequences and results. The strengths of each provide a more successful process than would normally be available using only ClustalW or HMMER alone. A more computationally efficient solution has been created to an interesting but complex computational problem.

\subsection{Data for Testing HMM approach}

To fully validate the software solution, sample data was used in a variety of experimental runs. The sample data served not only to assess the ability to identify primer candidates, but also to evaluate the performance of the software solution. The solution has been generalized for a variety of different circumstances. However, fungal data related to the specific projects of the university researchers mentioned previously provided a starting point and a means to evaluate the solution proposed.

Sample fungal data was collected from GenBank for use in validation of the proposed process. Sample data was stored in local BLAST databases, and retrieved for use in creation of HMMER profiles, and sequence alignments. Working with university researchers who were interested in identifying fungal diversity in soil samples, GenBank queries were modified and submitted in GenBank BLAST searches to collect and establish sample data. The queries used have been listed in the Appendices (Lord et al.). 
Focus was given to three fungal phyla - ascomycota, basidiomycota, and zygomycota.

Queries used to obtain fungal sequence data returned 66,197 ascomycota sequences, 36,078 basidiomycota sequences, and 1,567 zygomycota sequences. Chytridiomycota returned fewer than 800 sequences, and for this reason, was not considered for further review. The selection from ascomycota, basidiomycota and zygomycota provided a substantial group of sequence data. Some grouping or sub-selection was performed to simulate expected real world use.

\subsubsection{Use of existing primers to group sequences}

The use of two existing primers helped to group sequences into datasets appropriate for alignment and primer screening consideration. These are the forward primer, EF3RCNL 5' CAAACTTGGTCATTTAGAGGA $3^{\prime}$ and reverse primer ITS4 5' TCCTCCGCTTATTGATATGC 3'. Results from a BLAST query of each primer against each

of the local sequence databases (ascomycota, basidiomycota, and zygomycota) provided differing sequence datasets appropriate for testing purposes (Lord et al.).

\subsubsection{Sort Order}

Consideration was given to possible methods of ordering sequences, specifically the ordering of sequences based on sequence length, as input to ClustalW, with the view that different sequence orders would have the potential to impact the initial ClustalW alignments, and therefore, the HMMER training models and final sequence alignment for each sample group. Three sequence sort orders were chosen: (a) longest sequence first, with subsequent sequences descending in sequence length; (b) shortest sequence first, with subsequent sequences ascending in sequence 
length; and (c) an unsorted sequence dataset, listing sequences only in the order in which they were returned from internal BLAST queries. While ClustalW completes the alignment based on the guide tree, which should reflect relative phylogenic relationships within the sequence set, ClustalW would only be used for the first 100 or fewer sequences. There existed then the possibility that differing selections used as input to ClustalW, from the same larger data set, would influence the outcome of the ClustalW alignment, the HMMER profile, and the final HMMER consensus. A simple method of grouping sequences in different initial selections would be to base the first 100 sequences on relative length of sequences. In each dataset, the first 100 sequences would be input to ClustalW for the initial alignment, with the remaining sequences, up to an additional 400, were set aside as input to the HMMER alignment. But across each run of the experiment the same sequences were used in each test, only the order differed.

\subsubsection{Naming Convention for Identifying Sample Groups}

Sorting the sequences data meant that the same fungal phylum would appear as alignments and in results multiple times. A naming convention was established to label alignments, as well as the primer candidates, for identification in timings, primer analysis, and sequence alignment comparisons.

The final sequence batches have been abbreviated, using the initial primer match from the GenBank query (EF3RCNL or ITS4) of the sequence set ("E" or "I"), the fungal division ("A," "B" or "Z," representing the three fungal divisions selected), and the sort order ("L," "S" or "U" for long sequences first, short sequences first, or unsorted sequence order), to create a three character label, which could then be used for discussion purposes and to distinguish primer candidates from one another. 
As an example, "EAS," represents sequences that had matched the EF3RCNL primer, from within the ascomycota database, with the sequence grouping sorted shortest sequences first. Other sequence grouping labels were as shown in Table 1:

Table 1 - Naming Convention for Data and Primer Labels

\begin{tabular}{|l|l|l|l|l|}
\hline Primer Match & Sort Order & Ascomycota & Basidiomycota & Zygomycota \\
\hline EF3RCNL & & & & \\
\hline & Short & EAS & EBS & EZS \\
\hline & Long & EAL & EBL & EZL \\
\hline & Unsorted & EAU & EBU & EZU \\
\hline ITS4 & & & & \\
\hline & Short & IAS & IBS & IZS \\
\hline & Long & IAL & IBL & IZL \\
\hline & Unsorted & IAU & IBU & IZU \\
\hline
\end{tabular}


Table 2 below provides further detail regarding sequence data and sequence statistics, including average sequence length for each dataset. Data for Unsorted HMMER alignments is not shown, as these alignments proved unsuccessful in actual experimental runs.

Table 2 - Summary of Sequence Data Statistics for Each Dataset

\begin{tabular}{|c|c|c|c|c|c|}
\hline Sort Order & Database/Primer Grouping & $\begin{array}{l}\text { Alignment } \\
\text { Type }\end{array}$ & $\begin{array}{l}\text { Number of } \\
\text { Sequences }\end{array}$ & \begin{tabular}{|l|} 
Number of \\
Nucleotides
\end{tabular} & $\begin{array}{l}\text { Avg. Sequence } \\
\text { Length }\end{array}$ \\
\hline \multirow[t]{12}{*}{ Short } & Ascomycota- EF3RCNL & Clustal & 100 & 33433 & 334.33 \\
\hline & & HMM & 400 & 659371 & 1648.43 \\
\hline & Ascomycota- ITS4 & Clustal & 100 & 50536 & 505.36 \\
\hline & & HMM & 400 & 390343 & 975.86 \\
\hline & Basidiomycota - EF3RCNL & Clustal & 100 & 107045 & 1070.45 \\
\hline & & HMM & 400 & 745454 & 1863.64 \\
\hline & Basidiomycota - ITS4 & Clustal & 100 & 56250 & 562.50 \\
\hline & & HMM & 400 & 365641 & 914.10 \\
\hline & Zygomycota - EF3RCNL & Clustal & 100 & 102988 & 1029.88 \\
\hline & & HMM & 117 & 240948 & 2059.38 \\
\hline & Zygomycota - ITS4 & Clustal & 100 & 60772 & 607.72 \\
\hline & & HMM & 249 & 260061 & 1044.42 \\
\hline \multirow[t]{12}{*}{ Long } & Ascomycota- EF3RCNL & Clustal & 100 & 193482 & 1934.82 \\
\hline & & HMM & 400 & 499322 & 1248.31 \\
\hline & Ascomycota- ITS4 & Clustal & 100 & 154780 & 1547.80 \\
\hline & & $\mathrm{HMM}$ & 400 & 286099 & 715.25 \\
\hline & Basidiomycota - EF3RCNL & Clustal & 100 & 214842 & 2148.42 \\
\hline & & HMM & 400 & 637657 & 1594.14 \\
\hline & Basidiomycota - ITS4 & Clustal & 100 & 126440 & 1264.40 \\
\hline & & HMM & 400 & 295451 & 738.63 \\
\hline & Zygomycota - EF3RCNL & Clustal & 100 & 211097 & 2110.97 \\
\hline & & HMM & 117 & 132839 & 1135.38 \\
\hline & Zygomycota - ITS4 & Clustal & 100 & 155049 & 1550.49 \\
\hline & & HMM & 249 & 165784 & 665.80 \\
\hline \multirow[t]{11}{*}{ Unsorted } & Ascomycota- EF3RCNL & Clustal & 100 & 109361 & 1093.61 \\
\hline & & HMM & & & \\
\hline & Ascomycota- ITS4 & Clustal & 100 & 85028 & 850.28 \\
\hline & & HMM & & & \\
\hline & Basidiomycota - EF3RCNL & Clustal & 100 & 171856 & 1718.56 \\
\hline & & HMM & & & \\
\hline & Basidiomycota - ITS4 & Clustal & 100 & 95690 & 956.90 \\
\hline & & HMM & & & \\
\hline & Zygomycota - EF3RCNL & Clustal & 100 & 187203 & 1872.03 \\
\hline & & HMM & & & \\
\hline & Zygomycota - ITS4 & Clustal & 100 & 100912 & 1009.12 \\
\hline
\end{tabular}




\section{Experiment Description}

The following describes the steps followed in the experiment, to build alignments using ClustalW and HMMER, and to identify primer candidates.

\subsection{ClustalW Alignment}

An initial ClustalW alignment was generated using the first 100 sequences of each sample dataset. This alignment served as a training set for HMMER, to establish a profile or model specific to the sequence dataset. Default parameters for ClustalW were used in this experiment for each dataset.

\subsubsection{Trimming Alignments}

ClustalX, which enhances Clustal with a graphical user interface, allowed visual review and editing of the individual alignments, as suggested in the Clustal literature (Thompson, Higgins, and Gibson). Each ClustalW alignment was edited, removing individual sequences that had not aligned cleanly; removed were sequences that had introduced substantial gaps on either end of the alignment, while often not aligning successfully within the body of greater alignment. This suggested little genetic similarity between these organisms and the majority of the sequences represented in the alignment. No more than ten sequences per set were removed from each alignment; ten sequences was an artificial limitation imposed to ensure sequence datasets were edited in a similar manner, under similar constraints, given that editing itself introduced a subjective element to the process. Sequences selected for removal either were clearly extending gaps at either the beginning or the ending of the alignment, or constituted a visible mismatch when compared to the majority of sequences represented in the alignment. 
In the following example, Figure 8, a screen capture from ClustalX shows examples of sequences that would be selected for deletion from the initial ClustalW alignment. The first two selected sequences are mismatched when compared to the neighboring sequences. Their membership in the alignment is also shown to cause an unnecessary gap of eight to ten nucleotides. This gap would not otherwise be introduced at this point, if only the remaining sequences had been used to build the alignment. The last two sequences selected likewise show mismatches compared to the general alignment, and introduce a gap in the same region. Visually comparing the two sets of targeted sequences does not exhibit a strong match with one another, suggesting that they are not related, as well as being dissimilar to the majority of sequences.

Furthermore, within the same example, by removing the four sequences, approximately 540 gapped bases can be eliminated from the forward portion of the alignment, resulting in a more concentrated, dense alignment. Similar measures are taken with regard to the trailing extension of the alignment. This process was repeated for each initial ClustalW alignment, but was relatively simple to complete using the ClustalX tool, and only the most obvious sequences were selected for removal from the alignment. 


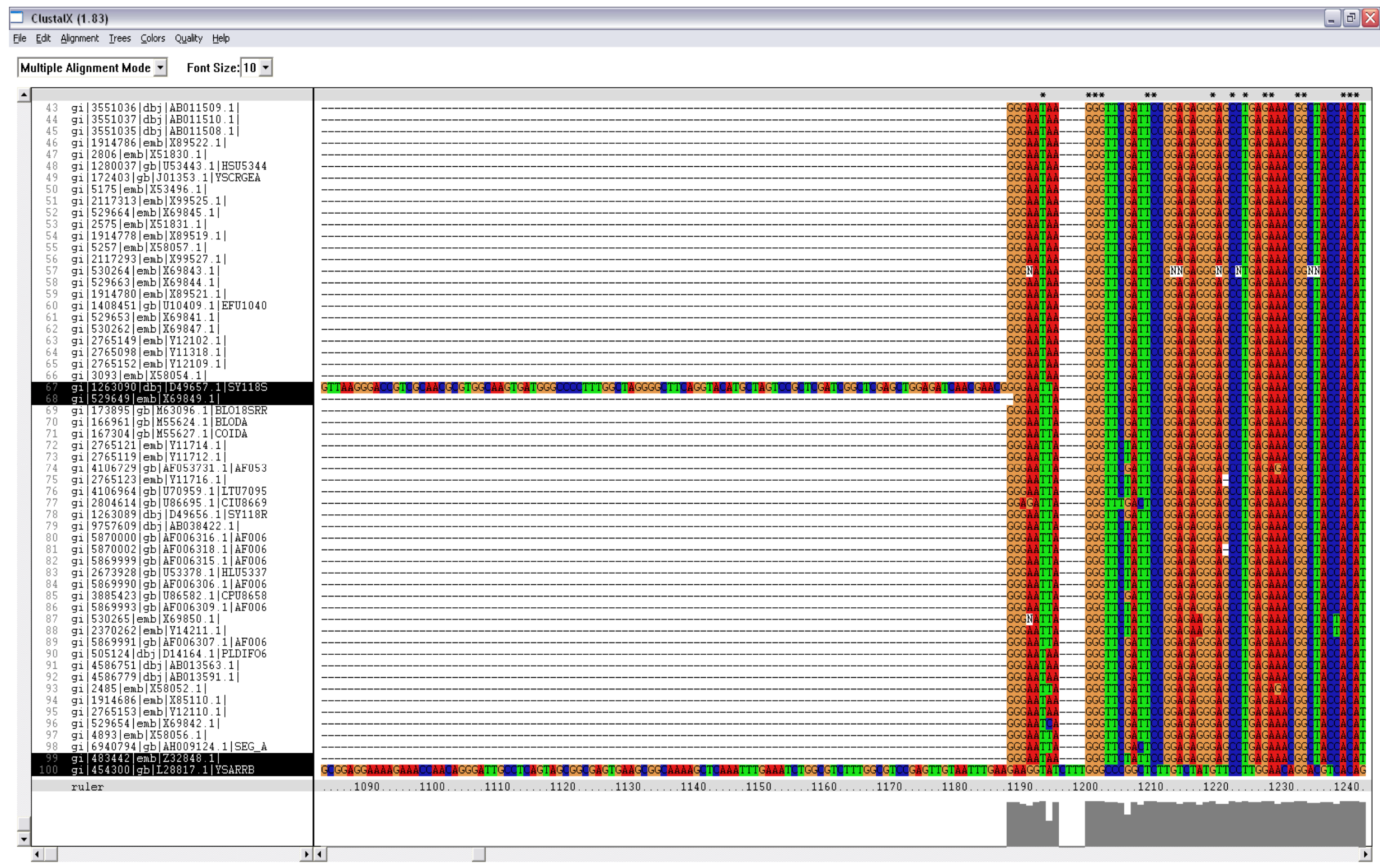

No color file found - using defaults

Figure 8 - Example of alignment editing, as viewed in ClustalX 


\subsection{HMMER Settings}

Default settings were used with Clustal, but within HMMER, the gap penalty was modified. Without this change, columns in the sequence alignment were observed to be shifted by one or two nucleotides for some sequences within the alignment, resulting in the appearance of a less conserved alignment, and reducing the quality of the overall consensus. While the potential of the alignment for success was observable in an alignment editor, the resulting HMMER alignment did not result in a clear consensus string. The specific flag, when used with the "hmmbuild" HMMER tool, is "--gapmax," which appeared to work best when set to " 0 .' The default value is 0.5 and the range of acceptable values is 0 to 1 . The intended effect of 'gapmax' is related to the inclusion of columns in the final alignment; set to ' 1 ', gap columns will be included in the consensus, while set to ' 0 ', the consensus will only include the aligned residues. The tool hmmbuild is used to create the HMMER profile from an existing alignment, such as from a previously completed ClustalW alignment.

\subsubsection{HMMER alignment and consensus}

No editing to the HMMER alignment was found to be necessary. It was useful, and relatively simple, to construct a consensus from the alignment. The HMMER tools can express a consensus, as mentioned before, using hmmemit, but it is a probability estimate, not an explicit consensus of the alignment. The emitted consensus strictly adhered to a statistical representation of the model, but for the purposes of this project, the result did not show all possibly conserved regions. The consensus has been 
calculated from the HMMER alignment using a script developed for this experiment. The character "." was used to designate introduction of gaps for the given position.

By default, nucleotides in ClustalW alignments are presented in upper case characters. HMMER ignores case, but by default, HMMER alignments represent aligned nucleotides in lower case; however, the character case of the input ClustalW alignment is preserved in the final HMMER alignment. In the consensus results presented in this experiment, upper case then shows data that had been aligned successfully in both the initial ClustalW alignment and in the HMMER alignment; that is to say, the data had not been aligned in ClustalW, only to be 'unaligned' in HMMER. Lower case segments represent data that was aligned successfully in the HMMER alignment, but had not been represented in the ClustalW alignment.

\subsection{Consensus Analysis and Identification of Primer Candidates}

Once the alignment had been completed, and a consensus produced, the software iterated over the consensus, checking for contiguous strings of nucleotides ranging in length from 17 to 24 bases. Melting temperature, GC content, and ending position for each string were also calculated and recorded. The reverse and reverse complement of each string is presented in the results as well, though GenBank BLAST searches for primer candidate returned the same results during validation, whether the primer is presented with the given forward presentation of a candidate or with a reverse-complement. As a convention, the primer candidates were labeled with their sample set name, e.g. EAS, and a number representing their ending position within the given consensus string. For example, primer candidate EAS_1065 would represent a primer candidate from the EAS 
sample data, located with ending position 1065 within the EAS alignment consensus. Ending position was chosen since primers of different lengths could potentially be selected from the same starting points, but their end points could still be differentiated. As a reminder, EAS is the sample data from the ascomycota dataset, sorted with short sequences presented first, that matched had initially matched the EF3RCNL primer.

\subsection{BLAST Candidates}

The selected primer candidates are provided as input to a Netblast search against GenBank non-redundant nucleotide database. The resulting report is saved as an html file for review, and as input for Netblast analysis, which provides a breakdown and count of the taxonomy portion of the BLAST results.

\subsection{Analysis of BLAST Hits}

For each primer candidate BLAST report, it was possible to summarize the taxonomy of the BLAST hits. While the default settings return a maximum of 500 hits, this sample result can provide an indication of specificity of the primer candidate for particular organisms.

\subsection{Test System Profile}

All tests, alignments, consensus calculations, GenBank BLAST runs, and taxonomy summarization were completed on a consumer desktop system, running Windows XP, with a Pentium $42.40 \mathrm{GHz}$ CPU, and 1.5 GB of RAM. Network connection was consumer DSL, with approximately $780 \mathrm{Kbps}$ connection speed. (Download Speed: $1543 \mathrm{kbps}(192.9 \mathrm{~KB} / \mathrm{sec}$ transfer rate) Upload Speed: $138 \mathrm{kbps}(17.3 \mathrm{~KB} / \mathrm{sec}$ transfer rate)). 


\subsection{Process for executing experiment using software interface}

The software interface developed as part of this project is primarily point-and-click, simplifying the process of working with the various underlying software components (ClustalW, ClustalX, HMMER, BLAST, and NetBLAST). This process is outlined in greater detail with accompanying illustrations in the appendix, though a summary is provided below.

\subsection{Process for executing experiment- Detailed View}

The process of selecting and testing primer candidates can be completed in nine basic steps, although expected use of the software would more likely cycle through subsets of select steps, particularly steps 4 through 9 , as shown below.

The procedures, corresponding to options presented in the user interface, are listed below, with a detailed explanation available in the Appendix:

1) Create BLAST Databases, using sequence data from GenBank

2) BLAST known primers to establish desired grouping of related sequence data

3) Retrieve fasta formatted sequences, according to particular filters

4) Create Clustal training alignment

5) Generate HMMER profile

6) Generate HMMER alignment and alignment consensus

7) Identify Primer candidates, using chosen technical constraints 
8) Run NetBLAST on selected primer candidates

9) Analyze NetBLAST matches for primer specificity

This process has three over-arching tasks. First, create a Clustal alignment, which will be used at a training alignment for the eventual HMMER profile and alignment. Second, create the HMMER profile and alignment. Third, generate primer candidates from the alignment consensus, and analyze the primer candidates for taxa relevancy.

Certain tasks are completed outside the software tool, in particular, the retrieval of GenBank fasta sequences that serve as alignment material, and therefore, aid in primer development. See Appendix for GenBank queries used, and process used to download GenBank files for use. 


\section{Experimental Results for HMMER Based Approach}

The tables below contain timing information for generating primer candidates, as well as the numbers of candidates generated. Changes between the different experiment runs are also described, focusing on how changes affected the outcome for candidate groups.

\subsection{Timings for Individual Portions of the Experiment}

This section shows timing results for specific steps within the process for determining primer candidates. Table 3 lists the amount of time required to create each of the local BLAST databases for each of the fungal divisions. The time needed for this operation is incidental, with the largest database needing less than five seconds to complete for this one-time operation, used to store the sample datasets. This is a one-time effort per data set, and does not contribute significantly to the overall process; there is no requirement to create a database, as a selection of sequences, properly gathered, can be submitted directly for processing.

Table 3 - Time to Create BLAST Databases

\begin{tabular}{|l|l|}
\hline \multicolumn{2}{|l|}{ Creating BLAST Databases } \\
\hline Database & Time to Complete (HH:MM:SS) \\
\hline Ascomycota & $0: 00: 04$ \\
\hline Basidiomycota & $0: 00: 03$ \\
\hline Zygomycota & $0: 00: 01$ \\
\hline
\end{tabular}


Table 4 lists time to complete the initial ClustalW alignment of 100 sequences or fewer.

Time to complete the alignment ranged from 33 seconds for the EAS group to over 28

minutes for the EBL group. Overall, timings were longer for the data set sorted with long

sequences first, as would be expected, since this represented an increased amount of

sequence data, if not by number of sequences, then by sequence length. Differences in

timings were most pronounced between the long and short sorted sequences; unsorted

data was closer in timing to the long-sorted data, than that of the short-sorted data.

Table 4 - Time to Complete ClustalW Alignments

ClustalW Alignment Timing Results - (HH:MM:SS)

\begin{tabular}{|c|c|c|c|}
\hline & \multicolumn{3}{|c|}{ Sort Order } \\
\hline $\begin{array}{l}\text { Sequence Group (<= } 100 \\
\text { Sequences) }\end{array}$ & Short & Long & Unsorted \\
\hline Ascomycota- EF3RCNL & $0: 00: 33$ & $0: 23: 46$ & $0: 06: 37$ \\
\hline Ascomycota- ITS4 & $0: 01: 19$ & 0:13:20 & $0: 03: 12$ \\
\hline Basidiomycota - EF3RCNL & 0:07:09 & $0: 28: 10$ & 0:20:01 \\
\hline Basidiomycota - ITS4 & $0: 01: 56$ & 0:09:21 & 0:04:36 \\
\hline Zygomycota - EF3RCNL & $0: 05: 16$ & $0: 27: 28$ & 0:23:59 \\
\hline Zygomycota - ITS4 & $0: 02: 22$ & $0: 11: 20$ & $0: 04: 25$ \\
\hline
\end{tabular}

Table 5 lists time necessary to create a new and unique HMMER profile for each

ClustalW alignment. These timings are incidental, with most completing in less than 1

second.

Table 5 - Time to Create HMMER Profile Based on Initial ClustalW Alignment

\begin{tabular}{|l|r|r|r|}
\hline \multicolumn{4}{|c|}{ HMMER Profile Creation - Timing Results - (HH:MM:SS) } \\
\hline \multicolumn{4}{|c|}{ Sort Order } \\
\hline Sequence Group & Short & Long & Unsorted \\
\hline & & & \\
\hline Ascomycota- EF3RCNL & $0: 00: 00$ & $0: 00: 00$ & $0: 00: 00$ \\
\hline Ascomycota- ITS4 & $0: 00: 00$ & $0: 00: 01$ & $0: 00: 01$ \\
\hline Basidiomycota - EF3RCNL & $0: 01: 25$ & $0: 00: 00$ & $0: 00: 00$ \\
\hline Basidiomycota - ITS4 & $0: 00: 00$ & $0: 00: 01$ & $0: 00: 01$ \\
\hline Zygomycota - EF3RCNL & $0: 00: 00$ & $0: 00: 00$ & $0: 00: 00$ \\
\hline Zygomycota - ITS4 & $0: 00: 00$ & $0: 00: 01$ & $0: 00: 01$ \\
\hline
\end{tabular}


Table 6 lists the amount of time required for completing the HMMER alignment, using up to 400 additional sequences against the previously created HMMER profile for each dataset. Though the HMMER alignments contained as many as four times the number of sequences as the initial ClustalW alignments, the longest runtime is slightly more than two minutes. Not all ClustalW datasets were usable as HMMER profiles and HMMER alignments. Though a higher throughput was shown, HMMER appears to require a higher degree of sequence identity than required by ClustalW to create an alignment.

Table 6 - Time to Complete HMMER Alignment

\begin{tabular}{|l|r|c|c|}
\hline \multicolumn{4}{|c|}{ HMMER Sequence Alignment - Timing Results - (HH:MM:SS) } \\
\hline \multicolumn{4}{|c|}{ Sort Order } \\
\hline Sequence Group & Short & Long & Unsorted \\
\hline & & & \\
\hline Ascomycota- EF3RCNL & $0: 00: 13$ & $0: 01: 47$ & No Alignment \\
\hline Ascomycota- ITS4 & $0: 00: 05$ & $0: 00: 29$ & No Alignment \\
\hline Basidiomycota - EF3RCNL & $0: 00: 05$ & $0: 02: 14$ & No Alignment \\
\hline Basidiomycota - ITS4 & $0: 00: 05$ & $0: 00: 18$ & No Alignment \\
\hline Zygomycota - EF3RCNL & $0: 00: 11$ & $0: 00: 19$ & No Alignment \\
\hline Zygomycota - ITS4 & $0: 00: 07$ & $0: 00: 06$ & No Alignment \\
\hline
\end{tabular}

For comparison, a baseline series of conventional alignments were created (Table 7), using only ClustalW, for datasets containing the same data as above, but in sequence sets that include 100,300 or 500 sequences.

Table 7 - ClustalW baseline alignment timings

\begin{tabular}{|l|r|r|r|}
\hline Conventional ClustalW Alignment - Timing Results - (HH:MM:SS) \\
\hline \multirow{2}{*}{$\begin{array}{l}\text { Sequence Group } \\
\text { (Sorted long to short) }\end{array}$} & \multicolumn{3}{|c|}{ Number of Sequences } \\
\cline { 2 - 4 } & 100 & 300 & 500 \\
\hline Ascomycota- ITS4 & $0: 13: 20$ & $0: 59: 54$ & $1: 28: 08$ \\
\hline Basidiomycota - EF3RCNL & $0: 28: 10$ & $3: 53: 32$ & $7: 13: 14$ \\
\hline
\end{tabular}




\subsection{Number of Primer Candidates Generated}

Analysis of the alignment consensus in some datasets produced more primer candidates

than expected, the largest listing over 1900 candidates. A possible problem may have been suggesting candidates of variable sizes as independent candidates. The initial settings may also have been too flexible, for example, allowing GC content in the range between $45 \%$ and $55 \%$, instead of simply adhering to a fixed value of $50 \%$ GC, and as such, the number of primer candidates needed to be manually reduced to a testable number of candidates for each of the datasets. The goal was to produce a list of ten viable primer candidates from each dataset, for use in both process and primer validation. Not all datasets were equally productive, as the smallest produced only two. In data sets where insufficient primers from independent regions could be identified, some flexibility was afforded and primer candidates were derived from overlapping conserved regions within the consensus. This required overlap in primer candidates' position within the consensus. From each dataset, manual selection from the list of available candidates was limited to candidates with $50 \%$ GC content. Priority was given to candidates that began or ended in combinations of CG. Candidates were selected from different areas in the consensus, where possible.

\subsection{HMMER parameters and effect on alignment}

Early sequence alignments had shown "shifts" in the HMMER alignment, related to inappropriate gap insertions, which were immediately visible in the resulting alignment, but initially difficult to interpret. The result was a degraded consensus, which inaccurately minimized conserved regions within the consensus output. Changing gap 
penalty values corrected new alignments of the same sequences, providing a richer consensus, with no apparent degradation to the alignment. This has been discussed earlier, in the comments related to the 'gapmax' parameter, used as an argument to the 'hmmbuild' command.

\subsection{Effect of using unsorted sequence data on alignment success}

Attempts to create alignments from the unsorted sequence samples were unsuccessful, and resulted in no usable HMMER alignments, and therefore, no primer candidates could be considered from this group. Since this grouping of sequences proved unreliable, it demonstrated sequence input order does impact eventual alignments, and the ability to create successful alignments, since the data in this set was the same as in the long-first sorted, and short-first sorted datasets; only the order differed. Difficulties creating alignments with unsorted data may reflect problems with gaps or other adjustments that cannot be modified once introduced. Lack of data similarity would be a potential explanation, except, as noted, the data is the same in each of the arrangements of sequences, only the sort order has changed.

Of note, much like the unsorted sequence sample, no successful HMMER alignment could be created using the EBS sample, with failure at the HMMER profile creation, and the sample set was not included in analysis for primer candidates. 


\section{Discussion}

\subsection{Timings for ClustalW and HMMER alignment processing}

The ClustalW alignment timings show impact of sort order on the length of time needed to complete the initial alignment; this is not surprising as the data sets sorted short-first contain fewer nucleotides to than those sorted long-first, reducing the complexity and amount of data to be aligned. This has a direct impact on the alignment process timing. For example, the difference between short-sorted sequence data and long-sorted sequence data, for the basidiomycota-EF3RNCL data set, was approximately 21 minutes, which in this case, demonstrates a four-fold increase in time (Table 4). For the same dataset, the HMMER alignment for the remaining 400 sequences (Table 6) shows a marked increase for the HMMER alignment of short-sorted data, which aligned in approximately five seconds, as compared to the HMMER alignment of long-sorted data, aligned in two minutes, 14 seconds. While the increase for HMMER appears proportionally more dramatic, both HMMER alignments still aligned 400 additional sequences in less time than Clustal aligned 100.

To further compare ClustalW and HMMER alignment times, the ClustalW alignment (Table 7) for basidiomycota data that had matched the EF3RCNL primer, and had been sorted long sequences first, completed in seven hours, 13 minutes. The same dataset aligned using the proposed solution architecture in just over 30 minutes; this includes the initial 28-plus minutes to create the ClustalW training alignment of 100 sequences, and the two-plus minutes to align in HMMER the remaining 400 sequences. It is expected 
that the solution architecture is able to limit the computational complexity of the HMMER alignment process to $\mathrm{O}(\mathrm{MN})$, by using a training alignment to begin the process. The ClustalW portion of the work is still subject to the range of computational complexity highlighted earlier, but the workload is bounded by a limit number of input sequences, mitigating some time and complexity.

\subsection{Organism Specificity of Primers Chosen}

There can be difficulty in assigning a subjective value to primer specificity, since this depends in part on the needs of the researcher; individual experiments may be satisfied by a primer that is no more specific than phylum level identification, while another experiment may need to be species-specific. Even within a given research effort, both types of primers may be necessary. The paper (Lord et. al.), which provided a starting point for this project, did not go deeper into taxa identification than phylum, in part because the diversity of sequence region being amplified had not yet been fully characterized. For the purposes of this experiment, the following valuation is proposed. Primers showing $100 \%$ specificity to a fungal phylum, based on the number of BLAST hits, can be considered specific to that phylum, and rated excellent. Those that show a greater than $90 \%$ match rate would be considered a good match to the given fungal phylum, while a match rate between $75 \%$ and $90 \%$ would be a fair match. Between $50 \%$ and $75 \%$, the primer would be showing a more general match to fungal organisms as well as other eukaryotes; in some cases, this might be the right primer for the research needs. Below 50\%, the primer may be considered too general for the purposes of this 
experiment, and primer candidates with no organism matches are not to be considered as primers.

From a computational perspective, a primer sequence has no intrinsic quality by which it can be identified with a particular organism taxon. Heuristics based on domain knowledge could be applied to enhance organism specific primer identification. Batch validation of primer candidates would also improve primer identification with regard to organism specificity.

Table 8 is a summary of BLAST hits for the primer candidates, matching kingdom "fungi," across each of the sample data sets. Of the 102 primer candidates, 56 returned $50 \%$ or greater BLAST hits for fungi, from the set of all BLAST hits, as a percentage of taxons of the kingdom level. A subset of 50 returned $75 \%$ or greater BLAST hits for fungi. Most queries returned 500 hits, though examples of returning fewer still can show matches for fungi for a given primer candidate.

Primer candidates representing both, regions aligned in ClustalW, represented in upper case characters, and those aligned in HMMER, represented in lower case characters, showed specificity for fungi organisms, though examples of ClustalW aligned and HMMER aligned primer candidates are shown to return no hits for fungi organisms. Primer candidates from datasets where sequences were sorted by length, short to long, are present in matches against fungi. The sort order of the sequences appear to have made less of a difference than the organism division dataset represented; this may have less to do with the organism than with the size or diversity of the original dataset. 
Table 8 - Summary of BLAST Hits for Primer Candidates with Fungal Specificity. Upper case characters represent ClustalW aligned data; lower case characters represent nucleotides aligned only in HMMER.

\begin{tabular}{|c|c|c|c|c|c|}
\hline $\begin{array}{l}\text { Sample } \\
\text { Alignment }\end{array}$ & \begin{tabular}{|l|}
$\begin{array}{l}\text { Primer } \\
\text { Candidate } \\
\text { Label }\end{array}$ \\
\end{tabular} & Primer Candidate & Taxon Matched & \begin{tabular}{|l} 
Number of \\
BLAST Hits
\end{tabular} & \begin{tabular}{|l|} 
Percent \\
Matching \\
Fungi \\
\end{tabular} \\
\hline \multirow[t]{10}{*}{ EAL } & EAL_724 & AACGGGTAACGGGGAATTAG & Fungi & 424 & $84.80 \%$ \\
\hline & EAL_771 & AAACGGCTACCACATCCAAG & Fungi & 163 & $32.60 \%$ \\
\hline & EAL_928 & AACGAGgAACAATTGGAGGG & Fungi & 477 & $95.40 \%$ \\
\hline & EAL_932 & AGgAACAATTGGAGGGCAAG & Fungi & 474 & $94.80 \%$ \\
\hline & EAL_936 & ACAATTGGAGGGCAAGTCTG & Fungi & 456 & $91.20 \%$ \\
\hline & EAL_1609 & AAGTTTTTGGGTTCTGGGGG & Fungi & 436 & $83.69 \%$ \\
\hline & EAL_1612 & TTTTTGGGTTCTGGGGGGAGTATG & Fungi & 425 & $82.21 \%$ \\
\hline & EAL_2205 & tgattttgtgggtggtggtG & Fungi & 483 & $96.60 \%$ \\
\hline & EAL_2207 & attttgtgggtggtggtGCATG & Fungi & 483 & $96.60 \%$ \\
\hline & EAL_2719 & TTGCGTTGATTACGTCCCTG & Fungi & 308 & $61.60 \%$ \\
\hline \multirow[t]{10}{*}{ EAS } & EAS_1065 & aaaaaaccettccggactcc & & 0 & $0.00 \%$ \\
\hline & EAS_1227 & ggagagggagggtgaaaaagaa & & 0 & $0.00 \%$ \\
\hline & EAS_1321 & aacaatatggggecctttcg & Fungi & 13 & $68.42 \%$ \\
\hline & EAS_1386 & aaaagtgggggaaaaagggg & & 0 & $0.00 \%$ \\
\hline & EAS_1557 & actttttcggggggttttgg & Fungi & 2 & $3.13 \%$ \\
\hline & EAS_1559 & tttttcggggggttttgggaaagg & Fungi & 1 & $3.23 \%$ \\
\hline & EAS_1560 & ttttcggggggttttgggaaag & Fungi & 3 & $6.82 \%$ \\
\hline & EAS_1874 & tttctcttagggtgtgggg & & 0 & $0.00 \%$ \\
\hline & EAS_2130 & cgagtggtgggggtaatttt & & 0 & $0.00 \%$ \\
\hline & EAS_2388 & CTTGTTAAACTCCGTCGTGCTG & Fungi & 483 & $93.42 \%$ \\
\hline \multirow[t]{10}{*}{ EBL } & EBL_362 & TTtcGATGGTAGGATAGAGGCC & Fungi & 391 & $78.20 \%$ \\
\hline & EBL_363 & TtcGATGGTAGGATAGAGGC & Fungi & 391 & $78.20 \%$ \\
\hline & EBL_443 & CTGAGAAACGGCTACCACAT & Fungi & 94 & $18.80 \%$ \\
\hline & EBL_1097 & AGAACGAAGGTTAGGGGATC & Fungi & 467 & $84.60 \%$ \\
\hline & EBL_1522 & TGCATGGCCGTTCTTAGTTGGTG & Fungi & 227 & $45.40 \%$ \\
\hline & EBL_1525 & ATGGCCGTTCTTAGTTGGTG & Fungi & 230 & $46.00 \%$ \\
\hline & EBL_1528 & GCCGTTCTTAGTTGGTGGAGTGAT & Fungi & 233 & $44.81 \%$ \\
\hline & EBL_1540 & TGGTGGAGTGATTTGTCTGg & Fungi & 188 & $32.47 \%$ \\
\hline & EBL_1987 & TGGctTAgTGAGATCTCCGGATTG & Fungi & 408 & $78.61 \%$ \\
\hline & EBL_1988 & GGctTAgTGAGATCTCCGGATT & Fungi & 397 & $76.94 \%$ \\
\hline \multirow[t]{2}{*}{ EZL } & EZL_2226 & TGGACTgGAATGAGGAATTC & & 0 & $0.00 \%$ \\
\hline & EZL_2227 & GGACTgGAATGAGGAATTC & & 0 & $0.00 \%$ \\
\hline \multirow[t]{8}{*}{ EZS } & EZS_2391 & ttgatgattttggcgggttggg & Fungi & 7 & $50.00 \%$ \\
\hline & EZS_2392 & tgatgattttggcgggttgg & Fungi & 6 & $66.67 \%$ \\
\hline & EZS_2483 & aggaagcaacgaaaacccaacc & Fungi & 5 & $23.81 \%$ \\
\hline & EZS_2486 & aagcaacgaaaacccaaccc & Fungi & 5 & $31.25 \%$ \\
\hline & EZS_3076 & agaagaaaggtgggggaaag & & 0 & $0.00 \%$ \\
\hline & EZS_3353 & CTTGAttCTATGGGTGGTGG & Fungi & 186 & $27.76 \%$ \\
\hline & EZS_3354 & TTGAttCTATGGGTGGTGGTGC & Fungi & 180 & $25.60 \%$ \\
\hline & EZS_3380 & CCGTTCTtAGTtGgTGGAGTgA & Fungi & 234 & $45.09 \%$ \\
\hline
\end{tabular}




\begin{tabular}{|c|c|c|c|c|c|}
\hline $\begin{array}{l}\text { Sample } \\
\text { Alignment }\end{array}$ & \begin{tabular}{|l}
$\begin{array}{l}\text { Primer } \\
\text { Candidate } \\
\text { Label }\end{array}$ \\
\end{tabular} & Primer Candidate & Taxon Matched & \begin{tabular}{|l} 
Number of \\
BLAST Hits
\end{tabular} & \begin{tabular}{|l|} 
Percent \\
Matching \\
Fungi \\
\end{tabular} \\
\hline & EZS_3389 & GTtGgTGGAGTgATTTGTCTGG & Fungi & 186 & $32.01 \%$ \\
\hline & EZS_3391 & tGgTGGAGTgATTTGTCTGG & Fungi & 188 & $32.47 \%$ \\
\hline & EZS_3415 & TTCCgATAACGAACGAgaCC & Fungi & 333 & $66.60 \%$ \\
\hline \multirow[t]{9}{*}{ IAL } & IAL_590 & TCGATGAAGAACGCAGCGAAaTGC & Fungi & 477 & $95.40 \%$ \\
\hline & IAL_591 & CGATGAAGAACGCAGCGAAa & Fungi & 485 & $97.00 \%$ \\
\hline & IAL_592a & GATGAAGAACGCAGCGAAaTGC & Fungi & 477 & $95.40 \%$ \\
\hline & IAL_592b & GATGAAGAACGCAGCGAAaTGCGA & Fungi & 477 & $95.40 \%$ \\
\hline & IAL_593 & ATGAAGAACGCAGCGAAaTGCG & Fungi & 478 & $95.60 \%$ \\
\hline & IAL_594 & TGAAGAACGCAGCGAAaTGC & Fungi & 478 & $95.60 \%$ \\
\hline & IAL_598 & GAACGCAGCGAAa TGCGATAAG & Fungi & 496 & $99.20 \%$ \\
\hline & IAL_600 & ACGCAGCGAAaTGCGATAAG & Fungi & 496 & $99.20 \%$ \\
\hline & IAL_601 & CGCAGCGAAaTGCGATAAGT & Fungi & 496 & $99.20 \%$ \\
\hline \multirow[t]{10}{*}{ IAS } & IAS_1845 & aaaccccccaattttccacagg & Fungi & 368 & $96.08 \%$ \\
\hline & IAS_1846 & aaccccccaattttccacag & Fungi & 352 & $97.51 \%$ \\
\hline & IAS_1949 & agaaacgcccattaaaccgg & Fungi & 1 & $50.00 \%$ \\
\hline & IAS_2047 & tgaagaacgcagcgaaatgC & Fungi & 478 & $95.60 \%$ \\
\hline & IAS_2053 & acgcagcgaaatgCGATAaG & Fungi & 496 & $99.20 \%$ \\
\hline & IAS_2173 & GAGCGtcatttcaaccctca & Fungi & 500 & $100.00 \%$ \\
\hline & IAS_2178 & tcatttcaaccctcaagcaccg & Fungi & 500 & $100.00 \%$ \\
\hline & IAS_2179 & catttcaaccctcaagcacc & Fungi & 500 & $100.00 \%$ \\
\hline & IAS_2288 & aaaagggaagcgcttgtgac & Fungi & 499 & $100.00 \%$ \\
\hline & IAS_2395 & agctagggaaaaagaccactcc & & 0 & $0.00 \%$ \\
\hline \multirow[t]{10}{*}{ IBL } & IBL_1991 & tgcttaggatgctggcgtaatg & Fungi & 500 & $100.00 \%$ \\
\hline & IBL_1994 & ttaggatgctggcgtaatgg & Fungi & 500 & $100.00 \%$ \\
\hline & IBL_2011 & tggctttaattgacccgtctgg & Fungi & 27 & $100.00 \%$ \\
\hline & IBL_2019 & attgacccgtctggaaacaagg & & 0 & $0.00 \%$ \\
\hline & IBL_2020 & ttgacccgtctggaaacaag & & 0 & $0.00 \%$ \\
\hline & IBL_2023 & acccgtctggaaacaaggacaaag & & 0 & $0.00 \%$ \\
\hline & IBL_2024 & cccgtctggaaacaaggacaaa & & 0 & $0.00 \%$ \\
\hline & IBL_2025 & ccgtctggaaacaaggacaa & & 0 & $0.00 \%$ \\
\hline & IBL_2067 & agttcgggtggaaaaccagtac & Fungi & 1 & $10.00 \%$ \\
\hline & IBL_2069 & ttcgggtggaaaaccagtac & & 0 & $0.00 \%$ \\
\hline \multirow[t]{10}{*}{ IBS } & IBS_363 & tgcgagtgaagtgggaatag & Fungi & 193 & $82.83 \%$ \\
\hline & IBS_366 & gagtgaagtgggaatagctC & Fungi & 9 & $29.03 \%$ \\
\hline & IBS_788 & gaaTGCaGctcaaaatgggtgg & Fungi & 500 & $100.00 \%$ \\
\hline & IBS_793 & CaGctcaaatgggtggtga & Fungi & 500 & $100.00 \%$ \\
\hline & IBS_839 & agagaccgatagcgaacaag & Fungi & 481 & $96.20 \%$ \\
\hline & IBS_959 & tcagccttgcttttgcttgg & Fungi & 500 & $100.00 \%$ \\
\hline & IBS_961 & agccttgcttttgcttggtg & Fungi & 500 & $100.00 \%$ \\
\hline & IBS_962 & gccttgcttttgcttggtgtac & Fungi & 500 & $100.00 \%$ \\
\hline & IBS_971 & ttgcttggtgtactttccgg & Fungi & 496 & $99.80 \%$ \\
\hline & IBS_1009 & ttttgaccgcgggataaagg & Fungi & 20 & $90.91 \%$ \\
\hline IZL & IZL_835a & ATGGATCTCTTGGTTCTCGC & Fungi & 500 & $100.00 \%$ \\
\hline
\end{tabular}




\begin{tabular}{|c|c|c|c|c|c|}
\hline $\begin{array}{l}\text { Sample } \\
\text { Alignment }\end{array}$ & \begin{tabular}{|l}
$\begin{array}{l}\text { Primer } \\
\text { Candidate } \\
\text { Label }\end{array}$ \\
\end{tabular} & Primer Candidate & Taxon Matched & \begin{tabular}{|l} 
Number of \\
BLAST Hits
\end{tabular} & \begin{tabular}{|l|} 
Percent \\
Matching \\
Fungi \\
\end{tabular} \\
\hline & IZL_835b & ATGGATCTCTTGGTTCTCGCATCG & Fungi & 500 & $100.00 \%$ \\
\hline & IZL_836 & TGGATCTCTTGGTTCTCGCATC & Fungi & 500 & $100.00 \%$ \\
\hline & IZL_837 & GGATCTCTTGGTTCTCGCAT & Fungi & 484 & $96.80 \%$ \\
\hline & IZL_839 & ATCTCTTGGTTCTCGCATCG & Fungi & 484 & $96.80 \%$ \\
\hline & IZL_840 & TCTCTTGGTTCTCGCATCGATG & Fungi & 484 & $96.80 \%$ \\
\hline & IZL_842 & TCTTGGTTCTCGCATCGATG & Fungi & 483 & $96.79 \%$ \\
\hline & IZL_1642 & atgtaaggaaggattgccgg & & 0 & $0.00 \%$ \\
\hline & IZL_1644 & gtaaggaaggattgccggattacc & Fungi & 1 & $11.11 \%$ \\
\hline & IZL_1646 & aaggaaggattgccggattacc & Fungi & 1 & $11.11 \%$ \\
\hline \multirow[t]{10}{*}{ IZS } & IZS_2495a & taggagatgatgggtatgcc & Fungi & 2 & $7.41 \%$ \\
\hline & IZS_2495b & taggagatgatgggtatgccag & Fungi & 5 & $12.82 \%$ \\
\hline & IZS_2501 & atgatgggtatgccagttatgggg & & 0 & $0.00 \%$ \\
\hline & IZS_2502 & tgatgggtatgccagttatggg & & 0 & $0.00 \%$ \\
\hline & IZS_2504 & atgggtatgccagttatggg & & 0 & $0.00 \%$ \\
\hline & IZS_2505 & tgggtatgccagttatggggtttg & & 0 & $0.00 \%$ \\
\hline & IZS_2506 & gggtatgccagttatggggttt & & 0 & $0.00 \%$ \\
\hline & IZS_2507 & ggtatgccagttatggggtt & & 0 & $0.00 \%$ \\
\hline & IZS_2511 & tgccagttatggggtttgaacg & & 0 & $0.00 \%$ \\
\hline & IZS_2512 & gccagttatggggtttgaac & & 0 & $0.00 \%$ \\
\hline
\end{tabular}

Table 9 summarizes primer specificity for the ascomycota division of fungal organisms within the ascomycota data set. Again, both ClustalW aligned regions as well as HMMER aligned regions have a demonstrated specificity to ascomycota fungal organisms, though the originating region that matched either the ITS4 primer or the EF3RCNL primer may show an effect on differing primer specificity. This effect is not shown in the same proportions in Table 10, which summarizes basidiomycota primer specificity. Primer candidates from the EAS alignment fared poorly, with few ClustalW aligned regions were available from which a primer candidate could be selected. This may indicate a widely diverse set of short segments, with little potential for alignment 
success. However, the single primer based on a ClustalW aligned region matched ascomycota for $99.41 \%$ at the phylum taxon level.

Table 9 - Summary of BLAST Hits for Primer Candidates with Specificity for Ascomycota. Upper case characters represent ClustalW aligned data; lower case characters represent nucleotides aligned only in HMMER.

\begin{tabular}{|c|c|c|c|c|c|c|c|}
\hline $\begin{array}{l}\text { Sample } \\
\text { Alignment }\end{array}$ & $\begin{array}{l}\text { Primer } \\
\text { Candidate } \\
\text { Label } \\
\end{array}$ & Primer Candidate & $\begin{array}{l}\text { Taxon } \\
\text { Matched }\end{array}$ & $\begin{array}{l}\text { Total } \\
\text { Number } \\
\text { of } \\
\text { BLAST } \\
\text { Hits } \\
\end{array}$ & $\begin{array}{l}\text { Taxon } \\
\text { Matched }\end{array}$ & $\begin{array}{l}\text { Total } \\
\text { Number } \\
\text { of } \\
\text { BLAST } \\
\text { Hits } \\
\end{array}$ & $\begin{array}{l}\text { Percent } \\
\text { Matching } \\
\text { Taxon } \\
\text { Level } \\
\end{array}$ \\
\hline \multirow[t]{10}{*}{ EAL } & EAL_724 & AACGGGTAACGGGGAATTAG & Fungi & 424 & Ascomycota & 206 & $65.40 \%$ \\
\hline & EAL_771 & AAACGGCTACCACATCCAAG & Fungi & 163 & Ascomycota & 52 & $10.70 \%$ \\
\hline & EAL_928 & AACGAGgAACAATTGGAGGG & Fungi & 477 & Ascomycota & 155 & $49.84 \%$ \\
\hline & EAL_932 & AGgAACAATTGGAGGGCAAG & Fungi & 474 & Ascomycota & 160 & $50.31 \%$ \\
\hline & EAL_936 & ACAATTGGAGGGCAAGTCTG & Fungi & 456 & Ascomycota & 160 & $47.06 \%$ \\
\hline & EAL_1609 & AAGTTTTTGGGTTCTGGGGG & Fungi & 436 & Ascomycota & 80 & $60.15 \%$ \\
\hline & EAL_1612 & TTTTTGGGTTCTGGGGGGAGTATG & Fungi & 425 & Ascomycota & 75 & $51.02 \%$ \\
\hline & EAL_2205 & tgattttgtgggtggtggtG & Fungi & 483 & Ascomycota & 277 & $97.54 \%$ \\
\hline & $\mathrm{EAL} 22207$ & attttgtgggtggtggtGCATG & Fungi & 483 & Ascomycota & 282 & $97.58 \%$ \\
\hline & EAL_2719 & TTGCGTTGATTACGTCCCTG & Fungi & 308 & Ascomycota & 19 & $8.96 \%$ \\
\hline \multirow[t]{10}{*}{ EAS } & EAS_1065 & Aaaaaccettccggactcc & & & & & \\
\hline & EAS_1227 & Ggagagggagggtgaaaaagaa & & & & & \\
\hline & EAS_1321 & Aacaatatggggccctttcg & Fungi & 13 & & & \\
\hline & EAS_1386 & Aaaagtgggggaaaaagggg & & 0 & & & \\
\hline & EAS_1557 & Actttttcggggggttttgg & Fungi & 2 & Ascomycota & 2 & $3.33 \%$ \\
\hline & EAS_1559 & Tttttcggggggttttgggaaagg & Fungi & 1 & Ascomycota & 1 & $2.70 \%$ \\
\hline & EAS_1560 & Ttttcggggggttttgggaaag & Fungi & 3 & & & \\
\hline & EAS_1874 & Tttctcttagggtgtgggg & & 0 & & & \\
\hline & EAS_2130 & Cgagtggtgggggtaatttt & & 0 & & & \\
\hline & EAS_2388 & CTTGTTAAACTCCGTCGTGCTG & Fungi & 483 & Ascomycota & 338 & $99.41 \%$ \\
\hline \multirow[t]{9}{*}{$\mathrm{IAL}$} & IAL_590 & TCGATGAAGAACGCAGCGAAaTGC & Fungi & 477 & Ascomycota & 379 & $77.19 \%$ \\
\hline & $\mathrm{IAL} 5591$ & CGATGAAGAACGCAGCGAAa & Fungi & 485 & Ascomycota & 364 & $74.44 \%$ \\
\hline & IAL_592a & GATGAAGAACGCAGCGAAaTGC & Fungi & 477 & Ascomycota & 379 & $77.35 \%$ \\
\hline & $\mathrm{IAL} \_592 \mathrm{~b}$ & GATGAAGAACGCAGCGAAaTGCGA & Fungi & 477 & Ascomycota & 379 & $77.35 \%$ \\
\hline & IAL_593 & ATGAAGAACGCAGCGAAaTGCG & Fungi & 478 & Ascomycota & 380 & $77.55 \%$ \\
\hline & IAL_594 & TGAAGAACGCAGCGAAaTGC & Fungi & 478 & Ascomycota & 380 & $77.55 \%$ \\
\hline & $\mathrm{IAL} \_598$ & GAACGCAGCGAAaTGCGATAAG & Fungi & 496 & Ascomycota & 401 & $82.34 \%$ \\
\hline & $\mathrm{IAL} \quad 600$ & ACGCAGCGAAaTGCGATAAG & Fungi & 496 & Ascomycota & 401 & $82.34 \%$ \\
\hline & $\mathrm{IAL} 6001$ & CGCAGCGAAaTGCGATAAGT & Fungi & 496 & Ascomycota & 401 & $82.34 \%$ \\
\hline \multirow[t]{3}{*}{ IAS } & IAS_1845 & Aaacccccaattttccacagg & Fungi & 368 & Ascomycota & 350 & $95.89 \%$ \\
\hline & IAS_1846 & Aaccceccaattttccacag & Fungi & 352 & Ascomycota & 334 & $97.38 \%$ \\
\hline & IAS_1949 & Agaaacgcccattaaaccgg & Fungi & 1 & & & \\
\hline
\end{tabular}




\begin{tabular}{|c|c|c|c|c|c|c|c|}
\hline $\begin{array}{l}\text { Sample } \\
\text { Alignment }\end{array}$ & $\begin{array}{l}\text { Primer } \\
\text { Candidate } \\
\text { Label }\end{array}$ & Primer Candidate & $\begin{array}{l}\text { Taxon } \\
\text { Matched }\end{array}$ & $\begin{array}{l}\text { Total } \\
\text { Number } \\
\text { of } \\
\text { BLAST } \\
\text { Hits } \\
\end{array}$ & $\begin{array}{l}\text { Taxon } \\
\text { Matched }\end{array}$ & $\begin{array}{l}\text { Total } \\
\text { Number } \\
\text { of } \\
\text { BLAST } \\
\text { Hits } \\
\end{array}$ & $\begin{array}{l}\text { Percent } \\
\text { Matching } \\
\text { Taxon } \\
\text { Level }\end{array}$ \\
\hline & IAS_2047 & tgaagaacgcagcgaaatgC & Fungi & 478 & Ascomycota & 380 & $77.55 \%$ \\
\hline & IAS_2053 & acgcagcgaaatgCGATAaG & Fungi & 496 & Ascomycota & 401 & $82.34 \%$ \\
\hline & IAS_2173 & GAGCGtcatttcaaccetca & Fungi & 500 & Ascomycota & 385 & $100.00 \%$ \\
\hline & IAS_2178 & Tcatttcaaccctcaagcaccg & Fungi & 500 & Ascomycota & 496 & $100.00 \%$ \\
\hline & IAS_2179 & Catttcaaccctcaagcacc & Fungi & 500 & Ascomycota & 496 & $100.00 \%$ \\
\hline & IAS_2288 & Aaaagggaagcgcttgtgac & Fungi & 499 & Ascomycota & 333 & $100.00 \%$ \\
\hline & IAS_2395 & Agctagggaaaaagaccactcc & & 0 & & & \\
\hline
\end{tabular}

Table 10 lists primer candidate specificity for fungal division basidiomycota, within the basidiomycota data set. Matches here showed less specificity, and in some cases, no matches at all, when compared to the ascomycota dataset. This may be due in part to the original data set size as the ascomycota data set, which showed higher specificity, contained nearly double the number of sequences as basidiomycota. Zygomycota, with a comparatively small data set, showed no primer specificity. Even though data set size contributes to the likelihood to proscribe primer candidates, special qualities about each data set, such as organism similarity, may play a more important role in primer identification. While we may propose a method for identifying primer candidates, those more familiar with the intrinsic qualities of the organism will be better qualified to understand biological factors in primer identification; these may include amount of existing data available for analysis, and the range of biological diversity within the dataset, suggesting a further narrowing of the dataset to more similar organisms. 
Table 10 - Summary of BLAST Hits for Primer Candidates with Specificity for Basidiomycota. Upper case characters represent ClustalW aligned data; lower case characters represent nucleotides aligned only in HMMER.

\begin{tabular}{|c|c|c|c|c|c|c|c|}
\hline $\begin{array}{l}\text { Sample } \\
\text { Alignment }\end{array}$ & $\begin{array}{l}\text { Primer } \\
\text { Candidate } \\
\text { Label }\end{array}$ & Primer Candidate & $\begin{array}{l}\text { Taxon } \\
\text { Matched }\end{array}$ & $\begin{array}{l}\text { Total } \\
\text { Number } \\
\text { of } \\
\text { BLAST } \\
\text { Hits }\end{array}$ & $\begin{array}{l}\text { Taxon } \\
\text { Matched }\end{array}$ & $\begin{array}{l}\text { Number } \\
\text { of } \\
\text { BLAST } \\
\text { Hits }\end{array}$ & $\begin{array}{l}\text { Percent } \\
\text { Matching } \\
\text { Taxon } \\
\text { Level }\end{array}$ \\
\hline \multicolumn{8}{|c|}{ Basidiomycota } \\
\hline \multirow[t]{10}{*}{ EBL } & EBL_362 & TTtcGATGGTAGGATAGAGGCC & Fungi & 391 & Basidiomycota & 123 & $36.07 \%$ \\
\hline & EBL_363 & TtcGATGGTAGGATAGAGGC & Fungi & 391 & Basidiomycota & 124 & $36.26 \%$ \\
\hline & EBL_443 & CTGAGAAACGGCTACCACAT & Fungi & 94 & Basidiomycota & 11 & $2.24 \%$ \\
\hline & EBL_1097 & AGAACGAAGGTTAGGGGATC & Fungi & 467 & Basidiomycota & 228 & $96.61 \%$ \\
\hline & EBL_1522 & TGCATGGCCGTTCTTAGTTGGTG & Fungi & 227 & Basidiomycota & 14 & $2.82 \%$ \\
\hline & EBL_1525 & ATGGCCGTTCTTAGTTGGTG & Fungi & 230 & Basidiomycota & 14 & $2.82 \%$ \\
\hline & EBL_1528 & GCCGTTCTTAGTTGGTGGAGTGAT & Fungi & 233 & Basidiomycota & 15 & $3.15 \%$ \\
\hline & EBL_1540 & TGGTGGAGTGATTTGTCTGg & Fungi & 188 & Basidiomycota & 14 & $3.35 \%$ \\
\hline & EBL_1987 & TGGctTAgTGAGATCTCCGGATTG & Fungi & 408 & Basidiomycota & 378 & $84.75 \%$ \\
\hline & EBL_1988 & GGctTAgTGAGATCTCCGGATT & Fungi & 397 & Basidiomycota & 376 & $78.17 \%$ \\
\hline EBS & & No Sample Data & & & & & \\
\hline \multirow[t]{10}{*}{$\mathrm{IBL}$} & IBL_1991 & Tgcttaggatgctggcgtaatg & Fungi & 500 & Basidiomycota & 451 & $99.78 \%$ \\
\hline & IBL_1994 & Ttaggatgctggcgtaatgg & Fungi & 500 & Basidiomycota & 442 & $98.66 \%$ \\
\hline & IBL_2011 & Tggctttaattgacccgtctgg & Fungi & 27 & Basidiomycota & 26 & $100.00 \%$ \\
\hline & IBL_2019 & Attgacccgtctggaaacaagg & & 0 & & & \\
\hline & IBL_2020 & Ttgacccgtctggaaacaag & & 0 & & & \\
\hline & IBL_2023 & Acccgtctggaaacaaggacaaag & & 0 & & & \\
\hline & IBL_2024 & cccgtctggaaacaaggacaaa & & 0 & & & \\
\hline & IBL_2025 & ccgtctggaaacaaggacaa & & 0 & & & \\
\hline & IBL_2067 & agttcgggtggaaaaccagtac & Fungi & 1 & Basidiomycota & 1 & $10.00 \%$ \\
\hline & IBL_2069 & ttcgggtggaaaaccagtac & & 0 & & & \\
\hline \multirow[t]{10}{*}{ IBS } & IBS_363 & tgcgagtgaagtgggaatag & Fungi & 193 & Basidiomycota & 122 & $68.54 \%$ \\
\hline & IBS_366 & gagtgaagtgggaatagctC & Fungi & 9 & & & \\
\hline & IBS_788 & gaaTGCaGctcaaaatgggtgg & Fungi & 500 & Basidiomycota & 381 & $81.41 \%$ \\
\hline & IBS_793 & CaGctcaaatgggtggtga & Fungi & 500 & Basidiomycota & 418 & $99.76 \%$ \\
\hline & IBS_839 & agagaccgatagcgaacaag & Fungi & 481 & Basidiomycota & 340 & $75.06 \%$ \\
\hline & IBS_959 & tcagccttgcttttgcttgg & Fungi & 500 & Basidiomycota & 466 & $99.79 \%$ \\
\hline & IBS_961 & agccttgcttttgcttggtg & Fungi & 500 & Basidiomycota & 465 & $99.57 \%$ \\
\hline & IBS_962 & gccttgcttttgcttggtgtac & Fungi & 500 & Basidiomycota & 469 & $100.00 \%$ \\
\hline & IBS_971 & ttgcttggtgtactttccgg & Fungi & 496 & Basidiomycota & 462 & $99.57 \%$ \\
\hline & IBS_1009 & ttttgaccgcgggataaagg & Fungi & 20 & Basidiomycota & 18 & $90.00 \%$ \\
\hline
\end{tabular}


The act of sorting sequences by length before alignment appears to directly affect the success of the alignment, with some impact on timing, and with an indirect impact on primer identification; in the solution proposed, a successful alignment is a prerequisite to primer identification. Unsorted data fared worse than data sorted by length. Data sorted by length, from long to short, may lend itself to alignment as longer sequences may allow for more overlap or similarity between organisms, if the data collected is otherwise unknown

\subsection{Software Interface as an Improvement to Process}

The software developed in this project offered a graphical user interface to applications that would normally be controlled through a command line interface. This can improve the flow of control for the researcher, highlighting potential next steps, though the choice is left to the user. A unified graphical interface may also simplify what can be a complex process. Each application accepts different parameters and arguments, as well as using differing data formats and file extensions for input and output data. The unifying software interface simplifies the process of sequence alignment, alignment review, and primer identification.

The software interface is not merely aesthetic. As part of its operation, scripts are invoked that sort the sequences before alignment occurs, parse consensus output for primer candidates while applying necessary primer constraints, and allow for batch processing for primer identification, and to some extent, batch processing for primer validation. 


\section{Conclusion}

Multiple sequence alignment software HMMER and ClustalW are fundamental tools in the field of sequence analysis. When used in combination they complement the algorithmic strengths of each. This technique can be effective in identifying conserved regions in large-scale sequence alignments, and serve as a successful means for identifying new primer candidates. The methods presented here are economical in terms of resource usage, offering an improved process for primer identification. Further work could more fully clarify the relationship of sequence sort order to alignment building, advance software interfaces, and further automate the primer identification and validation process.

In this project, samples of fungal data were aligned in two stages. Initially, a set of 100 sequences were aligned with ClustalW, and used as a foundation on which to build a larger sequence alignment. In the second stage, HMMER aligned a remaining set of up to 400 additional sequences, after building a statistical model from the smaller ClustalW alignment. Primers were identified from within the resulting consensus for select fungal divisions, with the understanding that larger sequence alignments may reflect conserved regions for particular taxa. The proposed primers were verified against the GenBank sequence database. Sample datasets had been sorted by sequence length, and were then used in multiple experimental runs.

Theoretically, HMMER would be expected to perform better than ClustalW based on the respective computational complexity for each software package, described earlier, with a worst case for HMMER of $\mathrm{O}\left(\mathrm{NM}^{2}\right)$ in time complexity, while ClustalW is shown to be 
$\mathrm{O}\left(\mathrm{N}^{4}+\mathrm{L}^{2}\right)$ for its final progressive alignment. Other steps in the ClustalW algorithm include computation of the distance matrix with time complexity $\mathrm{O}\left(\mathrm{N}^{2} \mathrm{~L}^{2}\right)$, and creation of the guide tree with time complexity $\mathrm{O}\left(\mathrm{N}^{3}\right)$.

Timings from experimental runs confirmed performance differences between ClustalW and HMMER, based on their differing approaches to sequence alignment. Baseline ClustalW alignments for 500 sequences were created, and ranged in time from 13 minutes, 20 seconds for 100 sequences to 7 hours, 13 minutes and 14 seconds for 500 sequences. Experimental timing runs in ClustalW, based on variations in sequence sort order, confirmed potential limitations of ClustalW with regard to performance. For example, entries in Table 4, listing time to complete initial ClustalW alignments, show the time needed to align 100 sequences can increase greatly, when the only change is a greater amount of sequence data by length. However, by limiting the task for ClustalW to creating a small but accurate training alignment, and shifting the greater sequence alignment burden to HMMER, such performance limitations are mitigated and the computational complexity for HMMER, as part of this solution, was held at $\mathrm{O}(\mathrm{MN})$. In the executing the experiment in this project, as much as a 15 -fold improvement in performance was shown, for the basidiomycota - EF3RCNL dataset, over a conventional Clustal alignment for the same dataset.

The effect of input data for ClustalW, specifically sorting the sequences by length, appears to have some limited impact on timing for the overall process, and also have significant impact on alignment quality. Short sequences appear more difficult to align 
to create a coherent consensus, and small datasets may prove difficult to use in this manner to identify primer candidates. HMMER is not able to align data that consists of short sequences that may not be directly connected over the space of the larger consensus. Where ClustalW may be able to align data that consists of shorter, less similar segments, gap introductions can reduce the overall quality of a ClustalW alignment consensus; though such gaps can be mitigated through a brief visual review of the alignment. A better approach might be to introduce heuristics that can adjust sequence data for sequences poorly matched for the overall alignment.

For best quality, the use of ClustalW to create a training alignment for use in HMMER is recommended. Performance is improved for the later HMMER alignment process, but alignment quality is improved, over using HMMER alone for multiple sequence alignments.

Sort order affects not only the time needed to align sequence data, but also the quality of the alignment and, indirectly, the ability to identify primer candidates. While this was hinted at by the authors of ClustalW, the impact was greater than had been expected, and would be recommended as a consideration for general alignment processing, and not only within the bounds of this proposed approach.

The solution outlined in this thesis was able to identify fungal specific primers; of those, primer candidates were introduced that showed a match greater than $90 \%$ for a given fungal phylum. 


\subsection{Future Work}

Additional research could explore further the impact of input data on alignment quality, in this case, defined as information hidden within the set of sequences that can be used to construct a consensus. The project had begun as an examination of how differing algorithmic methods could be combined to create a new approach to a given problem, but experimentation shows control and manipulation of input data may also play a part in the approach and solution. While performance goals were met, applicability was limited to those datasets that produced successful alignments.

Manual editing of the smaller alignment improved the quality of the eventual consensus. If this could be automated using basic heuristics, some improvement might be seen in the overall process. Future work could involve developing heuristics and measuring their impact on the application and quality of the result.

The application solution could benefit from better data management, most likely in the form of a results database, which could store alignments, consensus strings and primer candidates, with an optional form of notation or commentary for each. Batch validation for multiple primer candidates would also greatly enhance the process. 


\section{References}

Baxevanis, Andreas D., B. F. Francis Ouellette, Ed.. Bioinformatics: A Practical Guide to the Analysis of Genes and Proteins. Third Edition. Wiley-Interscience. 2005.

Black, Paul E., "hidden Markov model", in Dictionary of Algorithms and Data Structures [online], Paul E. Black, ed., U.S. National Institute of Standards and Technology. 14 August 2008.

Burpo, F. John. "A Critical Review of PCR Primer Design Algorithms and Crosshybridization.” 2001 : n. pag. Print.

Chenna, Ramu, Hideaki Sugawara, Tadashi Koike, Rodrigo Lopez, Toby J. Gibson, Desmond G. Higgins and Julie D. Thompson. "Multiple Sequence Alignment with the Clustal Series of Programs." Nucl. Acids Res. 31.13 (2003) : 3497-3500.

Churchill, G.A. "Stochastic Models for Heterogeneous DNA Sequences." Bulletin of Mathematical Biology Vol. 51 (1989) : 79-94. Print.

Eddy, S. R. "Hidden Markov Models and Large-Scale Genome Analysis." 1997. Print.

---. "Multiple Alignment Using Hidden Markov Models." AAAI Press, 1995. 114-120. Print.

---. "Profile Hidden Markov Models." Bioinformatics (Oxford, England) 14.9 (1998): 755-763. 
---. "HMMER User's Guide" (2003). HMMER Biological Sequence Analysis Using Profile Hidden Markov Modes (Version 2.3) [Software, Software Manual]. Available from http://hmmer.janelia.org/

Edgar, Robert. "MUSCLE: A Multiple Sequence Alignment Method with Reduced Time and Space Complexity.” BMC Bioinformatics 5.1 (2004) : 113. Print.

Kampke, Thomas, Markus Kieninger, and Michael Mecklenburg. "Efficient Primer Design Algorithms.” Bioinformatics 17.3 (2001) : 214-225.

Koski, Timo. Hidden Markov Modesl for Bioinformatics. Kluwer Academic Publishers, 2001. Print.

Lord, N. S., C.W. Kaplan, P. Shank, C.L. Kitts and S.L. Elrod. "Assessment of Fungal Diversity Using Terminal Restriction Fragment (TRF) Pattern Analysis: Comparison of 18S and ITS Ribosomal Regions." FEMS Microbiology Ecology 42.3 (2002) : 327-337.

Lowe, Todd, John Sharefkin, Shi Qi Yang, Carl W. Dieffenbach. “A Computer Program for Selection of Oligonucleotide Primers for Polymerase Chain Reactions." Nucleic Acids Research 18.7 (1990) : 1757-1761.

Loytynoja, Ari, and Michel C. Milinkovitch. "A Hidden Markov Model for Progressive Multiple Alignment.” Bioinformatics 19.12 (2003) : 1505-1513.

Misener, Stephen, and Stephen A. Krawetz, eds. Bioinformatics Methods and Protocols. Humana Press, 1999. Print. 
Notredame, Cédric. "Recent Progress in Multiple Sequence Alignment: A Survey." Pharmacogenomics 3.1 (2002) : 131-144.

Oliver, Tim, Bertil Schmidt, Darran Nathan, Ralf Clemens, Douglas Maskell. "Using Reconfigurable Hardware to Accelerate Multiple Sequence Alignment with ClustalW." Bioinformatics 21.16 (2005) : 3431-3432.

Thompson, Julie D., Desmond G. Higgins, and Toby J. Gibson. "CLUSTAL W: Improving the Sensitivity of Progressive Multiple Sequence Alignment Through Sequence Weighting, Position-specific Gap Penalties and Weight Matrix Choice.” Nucleic Acids Research 22.22 (1994) : 4673-4680.

Wang, L., and T. Jiang. "On the Complexity of Multiple Sequence Alignment." Journal of Computational Biology $\square$ : A Journal of Computational Molecular Cell Biology 1.4 (1994) : 337-348. Print. 


\section{Appendices}

\section{Primer Candidates}

Table 11 - List of Primer Candidates Evaluated and Primer Candidate Characteristics. Upper case characters represent ClustalW aligned data; lower case characters represent nucleotides aligned only in HMMER.

\begin{tabular}{|c|c|c|c|c|c|c|c|}
\hline & \begin{tabular}{|l|} 
Sample \\
Alignment
\end{tabular} & \begin{tabular}{|l|}
$\begin{array}{l}\text { Primer } \\
\text { Candidate } \\
\text { t Label }\end{array}$ \\
\end{tabular} & Primer Candidate & $\begin{array}{l}\text { Ending Position } \\
\text { within alignment }\end{array}$ & Melting Temperature & GC Content (\%) & Length \\
\hline 1 & EAL & EAL_724 & AACGGGTAACGGGGAATTAG & 724 & 60 & $50 \%$ & 20 \\
\hline 2 & & EAL_771 & AAACGGCTACCACATCCAAG & 771 & 60 & $50 \%$ & 20 \\
\hline 3 & & EAL_928 & AACGAGgAACAATTGGAGGG & 928 & 60 & $50 \%$ & 20 \\
\hline 4 & & EAL 932 & AGgAACAATTGGAGGGCAAG & 932 & 60 & $50 \%$ & 20 \\
\hline 5 & & EAL_936 & ACAATTGGAGGGCAAGTCTG & 936 & 60 & $50 \%$ & 20 \\
\hline 6 & & EAL_1609 & AAGTTTTTGGGTTCTGGGGG & 1609 & 60 & $50 \%$ & 20 \\
\hline 7 & & EAL_1612 & TTTTTGGGTTCTGGGGGGAGTATG & 1612 & 72 & $50 \%$ & 24 \\
\hline 8 & & EAL_2205 & tgattttgtgggtggtggt $G$ & 2205 & 60 & $50 \%$ & 20 \\
\hline 9 & & EAL_2207 & attttgtgggtggtggtGCATG & 2207 & 66 & $50 \%$ & 22 \\
\hline 10 & & EAL_2719 & TTGCGTTGATTACGTCCCTG & 2719 & 60 & $50 \%$ & 20 \\
\hline 11 & EAS & EAS_1065 & aaaaaaccettccggactcc & 1065 & 60 & $50 \%$ & 20 \\
\hline 12 & & EAS_1227 & ggagagggagggtgaaaaagaa & 1227 & 66 & $50 \%$ & 22 \\
\hline 13 & & EAS_1321 & aacaatatggggecctttcg & 1321 & 60 & $50 \%$ & 20 \\
\hline 14 & & EAS_1386 & aaaagtgggggaaaaagggg & 1386 & 60 & $50 \%$ & 20 \\
\hline 15 & & EAS_1557 & actttttcggggggttttgg & 1557 & 60 & $50 \%$ & 20 \\
\hline 16 & & EAS_1559 & tttttcggggggttttgggaaagg & 1559 & 72 & $50 \%$ & 24 \\
\hline 17 & & EAS_1560 & ttttcggggggttttgggaaag & 1560 & 66 & $50 \%$ & 22 \\
\hline 18 & & EAS_1874 & tttctctttagggtgtgggg & 1874 & 60 & $50 \%$ & 20 \\
\hline 19 & & EAS_2130 & cgagtggtgggggtaatttt & 2130 & 60 & $50 \%$ & 20 \\
\hline 20 & & EAS_2388 & CTTGTTAAACTCCGTCGTGCTG & 2388 & 66 & $50 \%$ & 22 \\
\hline 21 & EBL & EBL_362 & TTtCGATGGTAGGATAGAGGCC & 362 & 66 & $50 \%$ & 22 \\
\hline 22 & & EBL_363 & TtcGATGGTAGGATAGAGGC & 363 & 60 & $50 \%$ & 20 \\
\hline 23 & & EBL_443 & CTGAGAAACGGCTACCACAT & 443 & 60 & $50 \%$ & 20 \\
\hline 24 & & EBL_1097 & AGAACGAAGGTTAGGGGATC & 1097 & 60 & $50 \%$ & 20 \\
\hline 25 & & EBL_1522 & TGCATGGCCGTTCTTAGTTGGTG & 1522 & 70 & $52.17 \%$ & 23 \\
\hline 26 & & EBL_1525 & ATGGCCGTTCTTAGTTGGTG & 1525 & 60 & $50 \%$ & 20 \\
\hline 27 & & EBL_1528 & GCCGTTCTTAGTTGGTGGAGTGAT & 1528 & 72 & $50 \%$ & 24 \\
\hline 28 & & EBL_1540 & TGGTGGAGTGATTTGTCTGg & 1540 & 60 & $50 \%$ & 20 \\
\hline 29 & & EBL_1987 & TGGctTAgTGAGATCTCCGGATTG & 1987 & 72 & $50 \%$ & 24 \\
\hline 30 & & EBL_1988 & GGCtTAgTGAGATCTCCGGATT & 1988 & 66 & $50 \%$ & 22 \\
\hline 31 & EZL & EZL_2226 & TGGACTgGAATGAGGAATTC & 2226 & 58 & $45 \%$ & 20 \\
\hline 32 & & EZL_2227 & GGACTgGAATGAGGAATTC & 2227 & 56 & $47.37 \%$ & 19 \\
\hline 33 & EZS & EZS_2391 & ttgatgattttggcgggttggg & 2391 & 66 & $50 \%$ & 22 \\
\hline 34 & & EZS_2392 & tgatgattttggcgggttgg & 2392 & 60 & $50 \%$ & 20 \\
\hline
\end{tabular}




\begin{tabular}{|c|c|c|c|c|c|c|c|}
\hline & \begin{tabular}{|l} 
Sample \\
Alignmen
\end{tabular} & \begin{tabular}{|l|}
$\begin{array}{l}\text { Primer } \\
\text { Candidate } \\
\text { Label }\end{array}$ \\
\end{tabular} & Primer Candidate & $\begin{array}{l}\text { Ending Position } \\
\text { within alignment }\end{array}$ & Melting Temperature & GC Content $(\%)$ & Length \\
\hline 35 & & EZS_2483 & aggaagcaacgaaaacccaacc & 2483 & 66 & $50 \%$ & 22 \\
\hline 36 & & EZS_2486 & aagcaacgaaaacccaaccc & 2486 & 60 & $50 \%$ & 20 \\
\hline 37 & & EZS_3076 & agaagaaaggtgggggaaag & 3076 & 60 & $50 \%$ & 20 \\
\hline 38 & & EZS_3353 & CTTGAttCTATGGGTGGTGG & 3353 & 60 & $50 \%$ & 20 \\
\hline 39 & & EZS_3354 & TTGAttCTATGGGTGGTGGTGC & 3354 & 66 & $50 \%$ & 22 \\
\hline 40 & & EZS_3380 & CCGTTCTtAGTtGgTGGAGTgA & 3380 & 66 & $50 \%$ & 22 \\
\hline 41 & & EZS_3389 & GTtGgTGGAGTgATTTGTCTGG & 3389 & 66 & $50 \%$ & 22 \\
\hline 42 & & EZS_3391 & tGgTGGAGTgATTTGTCTGG & 3391 & 60 & $50 \%$ & 20 \\
\hline 43 & & EZS_3415 & TTCCgATAACGAACGAgaCC & 3415 & 60 & $50 \%$ & 20 \\
\hline 44 & IAL & IAL_590 & TCGATGAAGAACGCAGCGAAaTGC & 590 & 72 & $50 \%$ & 24 \\
\hline 45 & & IAL_591 & CGATGAAGAACGCAGCGAAa & 591 & 60 & $50 \%$ & 20 \\
\hline 46 & & IAL_592a & GATGAAGAACGCAGCGAAaTGC & 592 & 66 & $50 \%$ & 22 \\
\hline 47 & & IAL_592b & GATGAAGAACGCAGCGAAaTGCGA & 592 & 72 & $50 \%$ & 24 \\
\hline 48 & & IAL_593 & ATGAAGAACGCAGCGAAaTGCG & 593 & 66 & $50 \%$ & 22 \\
\hline 49 & & IAL_594 & TGAAGAACGCAGCGAAaTGC & 594 & 60 & $50 \%$ & 20 \\
\hline 50 & & IAL_598 & GAACGCAGCGAAaTGCGATAAG & 598 & 66 & $50 \%$ & 22 \\
\hline 51 & & IAL_600 & ACGCAGCGAAaTGCGATAAG & 600 & 60 & $50 \%$ & 20 \\
\hline 52 & & IAL_601 & CGCAGCGAAaTGCGATAAGT & 601 & 60 & $50 \%$ & 20 \\
\hline 53 & IAS & IAS_1845 & aaaccccccaattttccacagg & 1845 & 66 & $50 \%$ & 22 \\
\hline 54 & & IAS_1846 & aaccccccaattttccacag & 1846 & 60 & $50 \%$ & 20 \\
\hline 55 & & IAS_1949 & agaaacgcccattaaaccgg & 1949 & 60 & $50 \%$ & 20 \\
\hline 56 & & IAS_2047 & tgaagaacgcagcgaaatgC & 2047 & 60 & $50 \%$ & 20 \\
\hline 57 & & IAS_2053 & acgcagcgaaatgCGATAaG & 2053 & 60 & $50 \%$ & 20 \\
\hline 58 & & IAS_2173 & GAGCGtcatttcaaccctca & 2173 & 60 & $50 \%$ & 20 \\
\hline 59 & & IAS_2178 & tcatttcaaccctcaagcaccg & 2178 & 66 & $50 \%$ & 22 \\
\hline 60 & & IAS_2179 & catttcaaccctcaagcacc & 2179 & 60 & $50 \%$ & 20 \\
\hline 61 & & IAS_2288 & aaaagggaagcgcttgtgac & 2288 & 60 & $50 \%$ & 20 \\
\hline 62 & & IAS_2395 & agctagggaaaaagaccactcc & 2395 & 66 & $50 \%$ & 22 \\
\hline 63 & IBL & IBL_1991 & tgcttaggatgctggcgtaatg & 1991 & 66 & $50 \%$ & 22 \\
\hline 64 & & IBL_1994 & ttaggatgctggcgtaatgg & 1994 & 60 & $50 \%$ & 20 \\
\hline 65 & & IBL_2011 & tggctttaattgacccgtctgg & 2011 & 66 & $50 \%$ & 22 \\
\hline 66 & & IBL_2019 & attgacccgtctggaaacaagg & 2019 & 66 & $50 \%$ & 22 \\
\hline 67 & & IBL_2020 & ttgacccgtctggaaacaag & 2020 & 60 & $50 \%$ & 20 \\
\hline 68 & & IBL_2023 & acccgtctggaaacaaggacaaag & 2023 & 72 & $50 \%$ & 24 \\
\hline 69 & & IBL_2024 & cccgtctggaaacaaggacaaa & 2024 & 66 & $50 \%$ & 22 \\
\hline 70 & & IBL_2025 & ccgtctggaaacaaggacaa & 2025 & 60 & $50 \%$ & 20 \\
\hline 71 & & IBL_2067 & agttcgggtggaaaaccagtac & 2067 & 66 & $50 \%$ & 22 \\
\hline 72 & & IBL_2069 & ttcgggtggaaaaccagtac & 2069 & 60 & $50 \%$ & 20 \\
\hline 73 & IBS & IBS_363 & tgcgagtgaagtgggaatag & 363 & 60 & $50 \%$ & 20 \\
\hline 74 & & IBS_366 & gagtgaagtgggaatagctC & 366 & 60 & $50 \%$ & 20 \\
\hline 75 & & IBS_788 & gaaTGCaGctcaaaatgggtgg & 788 & 66 & $50 \%$ & 22 \\
\hline 76 & & IBS_793 & CaGctcaaaatgggtggtga & 793 & 60 & $50 \%$ & 20 \\
\hline 77 & & IBS_839 & lagagaccgatagcgaacaag & 839 & 60 & $50 \%$ & 20 \\
\hline
\end{tabular}




\begin{tabular}{|c|c|c|c|c|c|c|c|}
\hline & \begin{tabular}{|l} 
Sample \\
Alignment
\end{tabular} & \begin{tabular}{|l} 
Primer \\
Candidate \\
Label
\end{tabular} & Primer Candidate & $\begin{array}{l}\text { Ending Position } \\
\text { within alignment }\end{array}$ & Melting Temperature & GC Content (\%) & Length \\
\hline 78 & & IBS_959 & tcagccttgcttttgcttgg & 959 & 60 & $50 \%$ & 20 \\
\hline 79 & & IBS_961 & agccttgcttttgcttggtg & 961 & 60 & $50 \%$ & 20 \\
\hline 80 & & IBS_962 & gccttgcttttgcttggtgtac & 962 & 66 & $50 \%$ & 22 \\
\hline 81 & & IBS_971 & ttgcttggtgtactttccgg & 971 & 60 & $50 \%$ & 20 \\
\hline 82 & & IBS_1009 & ttttgaccgcgggataaagg & 1009 & 60 & $50 \%$ & 20 \\
\hline 83 & IZL & IZL_835a & ATGGATCTCTTGGTTCTCGC & 835 & 60 & $50 \%$ & 20 \\
\hline 84 & & IZL_835b & ATGGATCTCTTGGTTCTCGCATCG & 835 & 72 & $50 \%$ & 24 \\
\hline 85 & & IZL_836 & TGGATCTCTTGGTTCTCGCATC & 836 & 66 & $50 \%$ & 22 \\
\hline 86 & & IZL_837 & GGATCTCTTGGTTCTCGCAT & 837 & 60 & $50 \%$ & 20 \\
\hline 87 & & IZL_839 & ATCTCTTGGTTCTCGCATCG & 839 & 60 & $50 \%$ & 20 \\
\hline 88 & & IZL_840 & TCTCTTGGTTCTCGCATCGATG & 840 & 66 & $50 \%$ & 22 \\
\hline 89 & & IZL_842 & TCTTGGTTCTCGCATCGATG & 842 & 60 & $50 \%$ & 20 \\
\hline 90 & & IZL_1642 & atgtaaggaaggattgccgg & 1642 & 60 & $50 \%$ & 20 \\
\hline 91 & & IZL_1644 & gtaaggaaggattgccggattacc & 1644 & 72 & $50 \%$ & 24 \\
\hline 92 & & IZL_1646 & aaggaaggattgccggattacc & 1646 & 66 & $50 \%$ & 22 \\
\hline 93 & IZS & IZS_2495a & taggagatgatgggtatgcc & 2495 & 60 & $50 \%$ & 20 \\
\hline 94 & & IZS_2495b & taggagatgatgggtatgccag & 2495 & 66 & $50 \%$ & 22 \\
\hline 95 & & IZS_2501 & atgatgggtatgccagttatgggg & 2501 & 72 & $50 \%$ & 24 \\
\hline 96 & & IZS_2502 & tgatgggtatgccagttatggg & 2502 & 66 & $50 \%$ & 22 \\
\hline 97 & & IZS_2504 & atgggtatgccagttatggg & 2504 & 60 & $50 \%$ & 20 \\
\hline 98 & & IZS_2505 & tgggtatgccagttatggggtttg & 2505 & 72 & $50 \%$ & 24 \\
\hline 99 & & IZS_2506 & gggtatgccagttatggggttt & 2506 & 66 & $50 \%$ & 22 \\
\hline 100 & & IZS_2507 & ggtatgccagttatggggtt & 2507 & 60 & $50 \%$ & 20 \\
\hline 101 & & IZS_2511 & tgccagttatggggtttgaacg & 2511 & 66 & $50 \%$ & 22 \\
\hline 102 & & IZS_2512 & gccagttatggggtttgaac & 2512 & 60 & $50 \%$ & 20 \\
\hline
\end{tabular}




\section{GenBank Queries}

Queries used to collect and establish sample data

(fungal OR fungi OR fungus) AND (ribosomal OR ribosome OR 18s OR ITS OR 28s OR 25s OR 5.8 OR "internal transcribed spacer") NOT "partial genome" NOT "complete genome" NOT "complete mitochondria genome" NOT "cosmid" NOT "chromosome" chytridiomycota AND (fungal OR fungi OR fungus) AND (ribosomal OR ribosome OR 18s OR ITS OR 28s OR 25s OR 5.8 OR "internal transcribed spacer") NOT "partial genome" NOT "complete genome" NOT "complete mitochondria genome" NOT "cosmid" NOT "chromosome"

ascomycota AND (fungal OR fungi OR fungus) AND (ribosomal OR ribosome OR 18s OR ITS OR 28s OR 25s OR 5.8 OR "internal transcribed spacer") NOT "partial genome" NOT "complete genome" NOT "complete mitochondria genome" NOT "cosmid" NOT "chromosome"

basidiomycota AND (fungal OR fungi OR fungus) AND (ribosomal OR ribosome OR 18s OR ITS OR 28s OR 25s OR 5.8 OR "internal transcribed spacer") NOT "partial genome" NOT "complete genome" NOT "complete mitochondria genome" NOT "cosmid" NOT "chromosome"

zygomycota AND (fungal OR fungi OR fungus) AND (ribosomal OR ribosome OR 18s OR ITS OR 28s OR 25s OR 5.8 OR "internal transcribed spacer") NOT "partial genome" NOT "complete genome" NOT "complete mitochondria genome" NOT "cosmid" NOT "chromosome"

\section{Baseline Primers}

Primers

forward-EF3RCNL 5'CAAACTTGGTCATTTAGAGGA 3'

reverse-ITS4 5'TCCTCCGCTTATTGATATGC 3' 


\section{Viterbi Algorithms}

1) Viterbi training algorithm, related to generation of HMM profiles

"An approach to model parameter estimation is the Viterbi training algorithm. In this approach, the most probable internal state sequence (path) associated to each observed sequence is derived using the Viterbi decoding algorithm. Then this path is used for estimating counts for the number of transitions and emissions, and such counts are used for recalculating the model parameters."

(De Fonzo, et. al.)

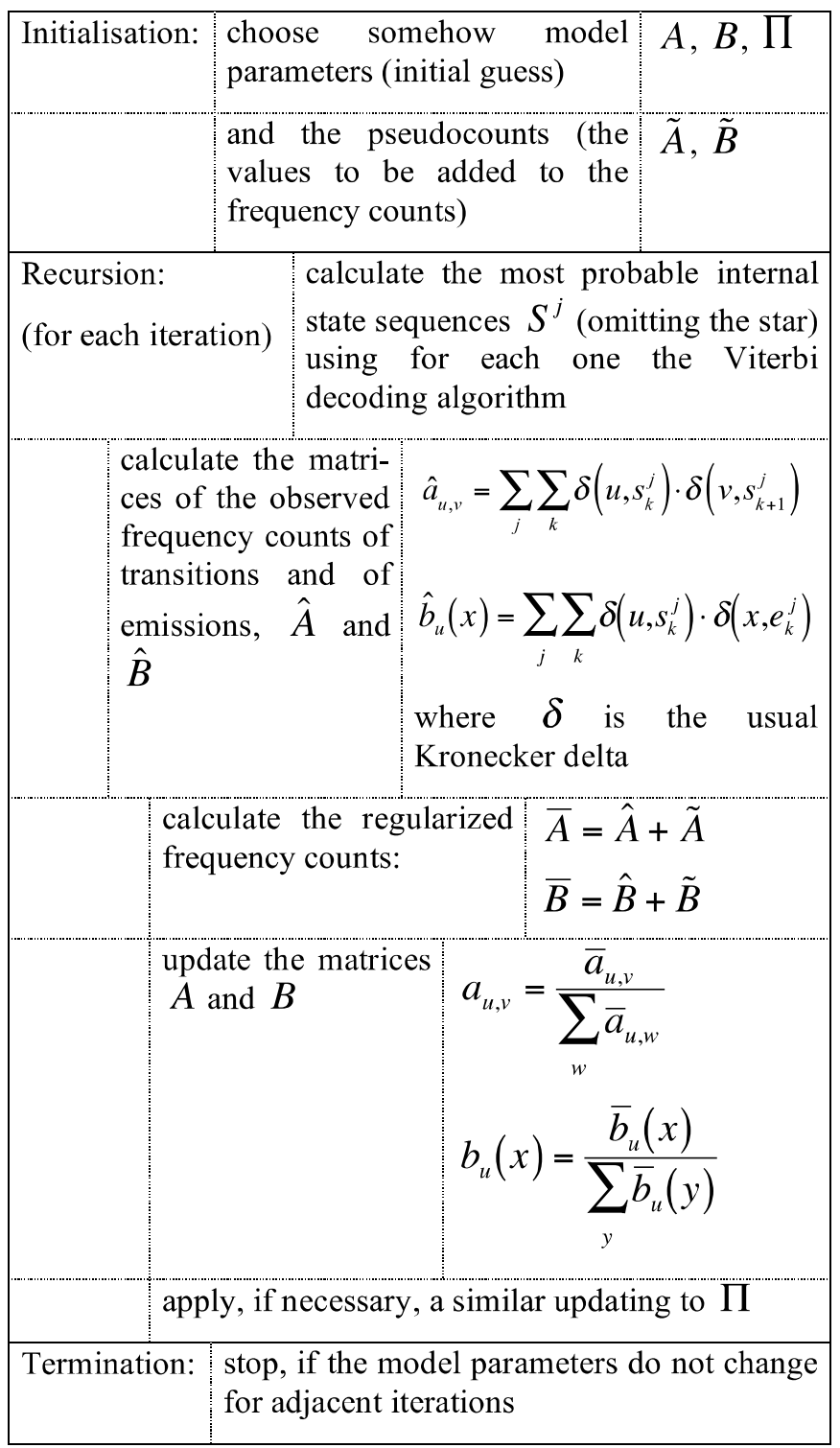


2) Viterbi algorithm, as used for sequence alignment in conjunction with an existing HMM profile

"In general terms, a problem of this type is to induce the most likely hidden states given a model and a sequence of observations. The two most common problems of this type, each one requiring an appropriate algorithm, are detailed in the next two paragraphs."

(De Fonzo, et. al.)

The Viterbi algorithm solves the following decoding problem.

Given a model $\lambda$ and a sequence $E$ of observed states, find the sequence $S^{*}$ of internal states that maximises the probability $P(E, S \mid \lambda)$, i.e. the sequence $S^{*}$ such that $p^{*} \equiv P\left(E, S^{*} \mid \lambda\right) \equiv \max _{S}(P(E, S \mid \lambda))$ or, more briefly, $S^{*} \equiv \arg \max (P(E, S \mid \lambda))$

..."'a sequence of internal states can be represented as a path; and the DP method applied to path optimization includes two successive phases: a first phase optimizes a number of sub problems, by storing suitable pointers that indicate promising (suboptimal) state transitions, and a second (reverse) phase obtains the optimal path by following the pointers. Detailed equations follow."

\begin{tabular}{|l|l|}
\hline Initialisation: & $\gamma_{1}(u)=b_{u}\left(e_{1}\right) \cdot \pi_{u}$ \\
& $\psi_{1}(u)=0$ \\
\hline $\begin{array}{l}\text { Recursion: } \\
\text { for } 1<k \leq L)\end{array}$ & $\gamma_{k}(u)=b_{u}\left(e_{k}\right) \cdot \max _{v}\left(\gamma_{k-1}(v) \cdot a_{v, u}\right)$ \\
& $\psi_{k}(u)=\underset{v}{\operatorname{argmax}}\left(\gamma_{k-1}(v) \cdot a_{v, u}\right)$ \\
\hline Termination: & $p^{*}=\underset{v}{\max }\left(\gamma_{L}(v)\right)$ \\
\hline $\begin{array}{l}\text { Backtracking: } \\
\text { (for } L>k \geq 1)\end{array}$ & $s_{L}^{*}=\underset{v}{\arg \max }\left(\gamma_{L}(v)\right)$ \\
\hline
\end{tabular}




\section{Process for executing experiment - Detailed View of Software Use}

The process of selecting and testing primer candidates can be completed in nine basic steps, although expected use of the software would more likely cycle through subsets of select steps, particularly steps 4 through 9 , as shown below.

The procedures, corresponding to options presented in the user interface, are listed below, with explanation and objectives of each to follow:

1) Create BLAST Databases, using sequence data from GenBank

2) BLAST known primers to establish desired grouping of related sequence data

3) Retrieve fasta formatted sequences, according to particular filters

4) Create Clustal training alignment

5) Generate HMMER profile

6) Generate HMMER alignment and alignment consensus

7) Generate Primer candidates, using chosen technical constraints

8) Run NetBLAST on selected primers candidates

9) Analyze NetBLAST result for primer specificity

This process has three over-arching tasks. First, create a Clustal alignment, which will be used at a training alignment for the eventual HMMER profile and alignment. Second, create the HMMER profile and alignment. Third, generate primer candidates from the alignment consensus, and analyze the primer candidates for taxa relevancy.

Certain tasks are completed outside the software tool, in particular, the retrieval of GenBank fasta sequences that serve as alignment material, and therefore, aid in primer development. See Appendix for GenBank queries used, and process used to download GenBank files for use. 
1. Create BLAST Databases, using fungal data from GenBank

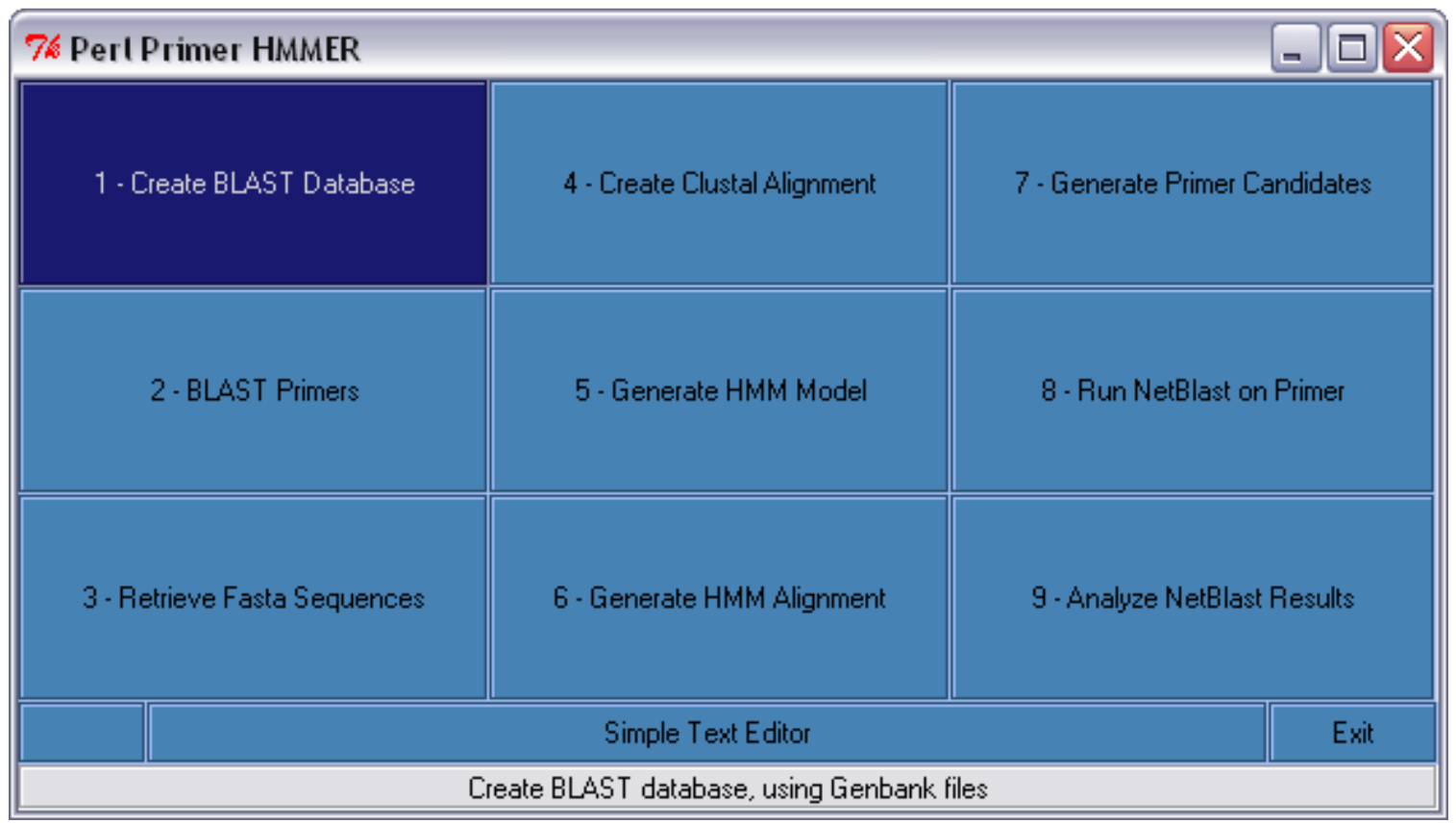

Figure 9 - Software interface - Step 1 "Create BLAST Database"

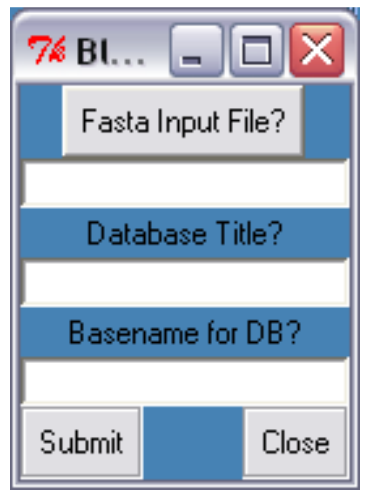

Figure 10 - Input Options for Creating BLAST Database

Having the GenBank files in place, a local blast database can be created, specific to the organism for which the primers will be designed. Selecting the button labeled "Create BLAST Database," the user is prompted for the input file, the title that will be used for the new database, and a basename for the database. Once the fields have been populated, and the user submits their request, and "formatdb", a blast database creation utility, is run, with the following options. The title for the database files, the input file, a log file of the database creation, $-\mathrm{p} \mathrm{F}$, signifying that it is a nucleotide database and $-\mathrm{o}$, which forces formatdb to parse the sequence ids and create indexes. 
2. BLAST known primers to establish desired sequence range

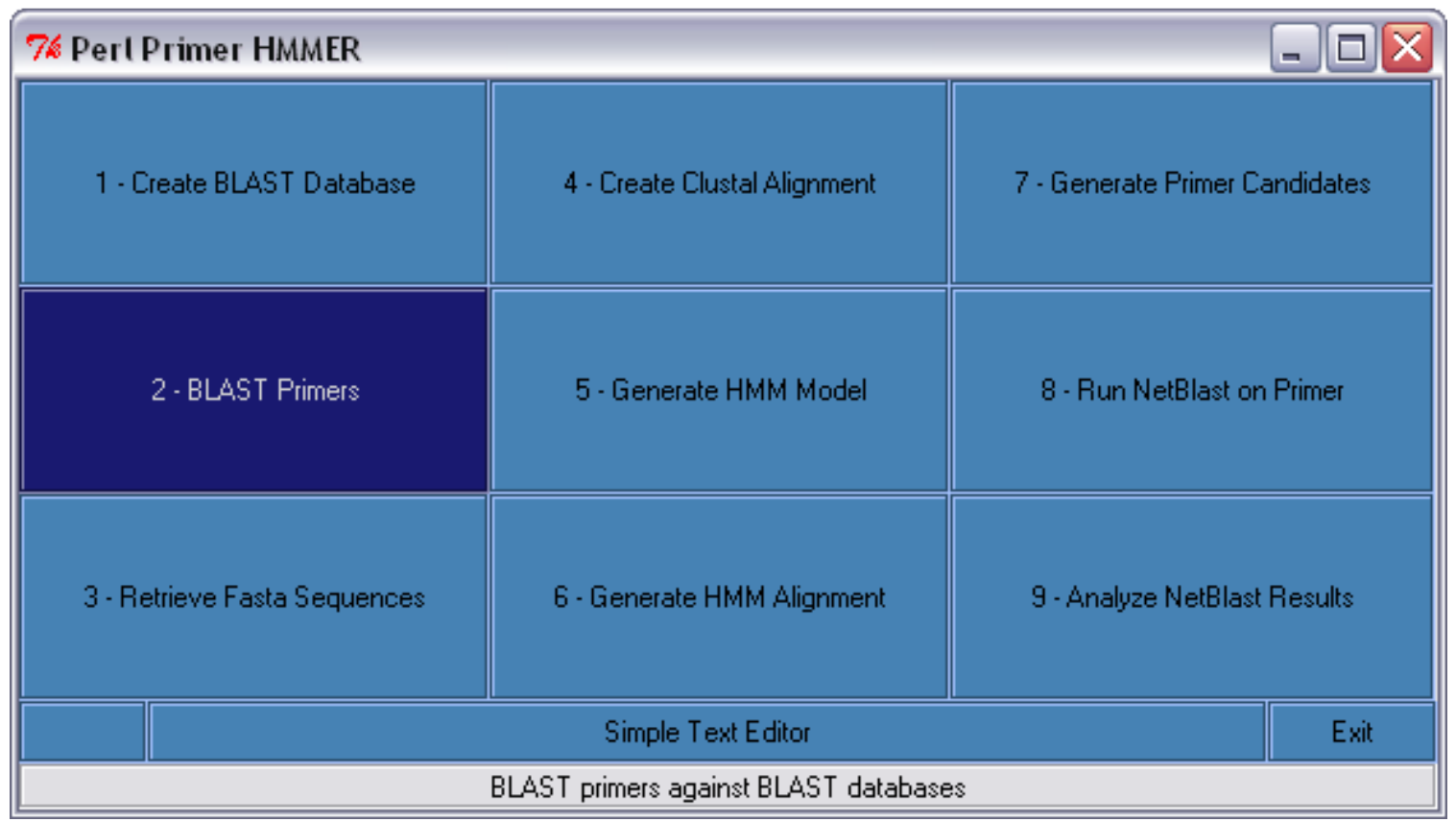

Figure 11 - BLAST Primers

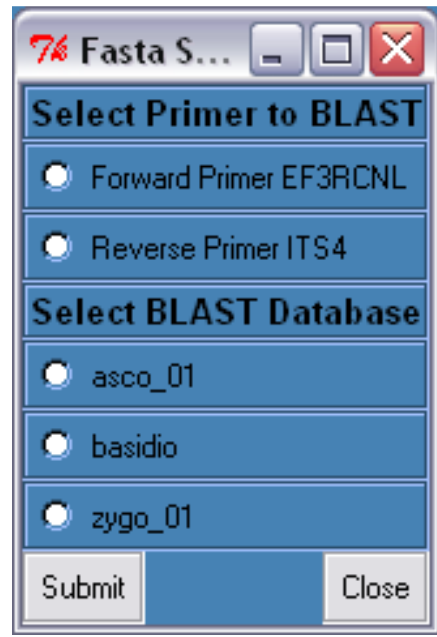

Figure 12 - Primer Selection for BLAST Processing

At this point, the new fasta database is now ready for use. In this case, the reference primers will identify sequences to be used in the later Clustal and HMMER alignments. The user is prompted to choose one of either the current forward or reverse primers, EF3RCNL or ITS4. Further prompts requests a choice of BLAST database to use. Once chosen and submitted, the user's request runs "blastn" against the selected database, with the given primer as input, and generates output, \{Comment: is this correct? a file containing sequence data, automatically named according to the matched primer name and database name, with a .txt extension. 
3. Retrieve fasta formatted sequences, according to particular filters

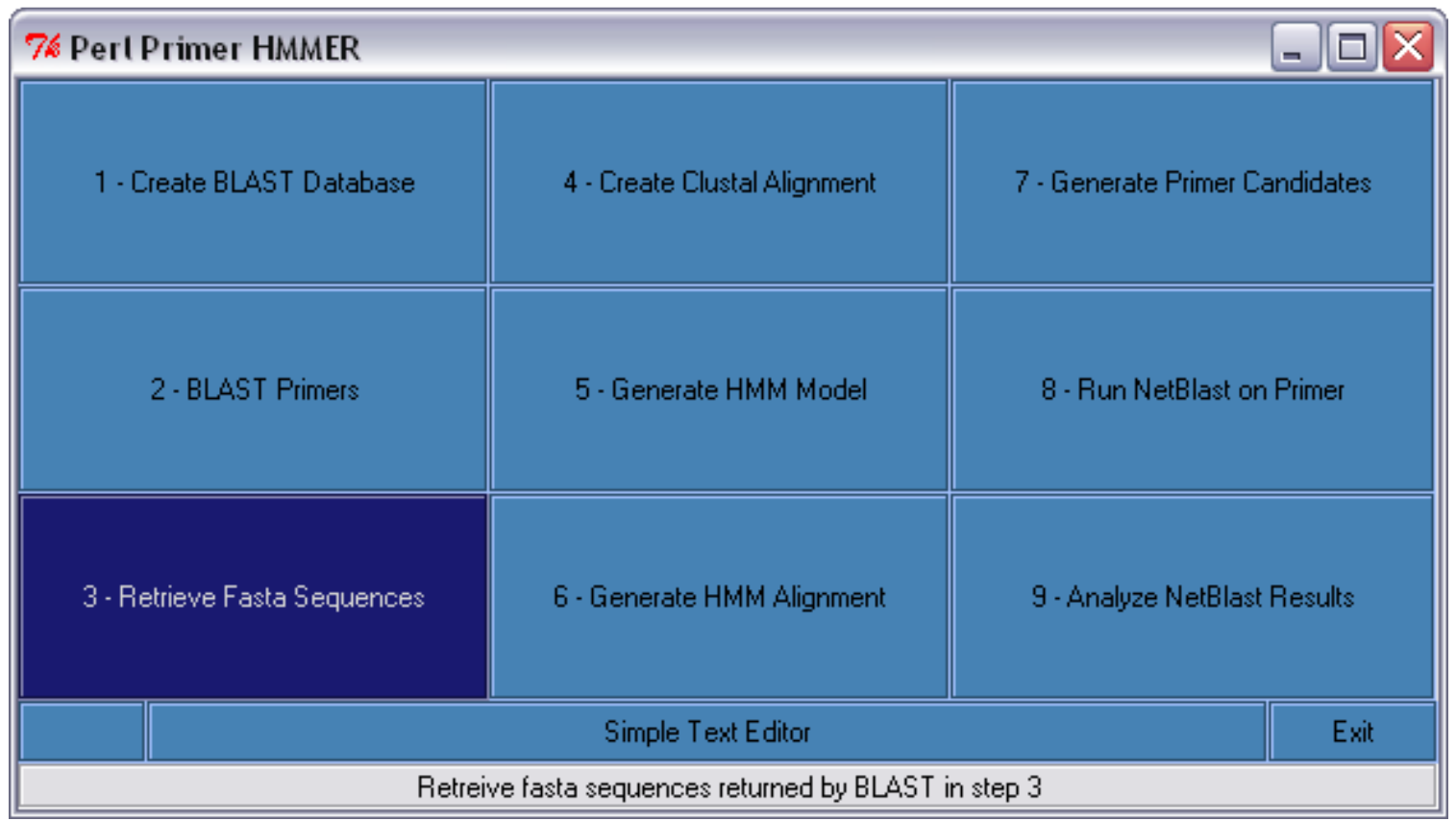

Figure 13 - Retrieve Matching Fasta Formatted Sequences

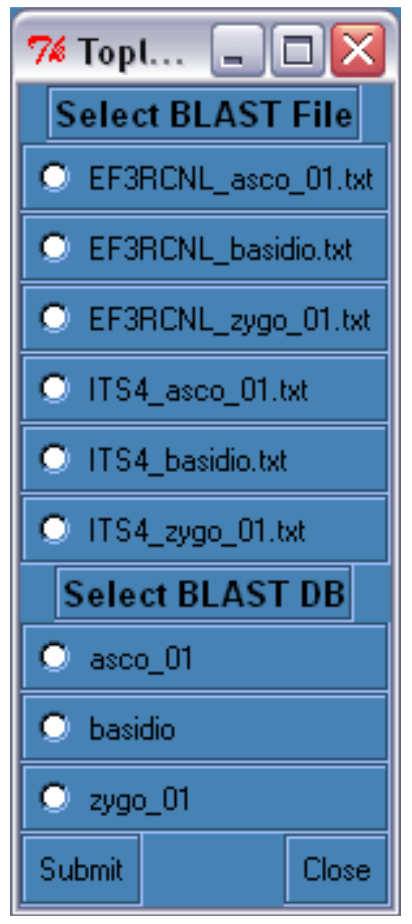

Figure 14 - Select BLAST DB and Fasta Sequences for Sorting

The blast report contains a listing of matches. The next step is to extract the matching sequences into files usable for the Clustal and HMMER alignments. The user is prompted 
for the BLAST file and the BLAST database. From there, once submitted, the software extracts the sequences, sorts the sequences by sequence length, from longest to shortest. The software then outputs the sequence information to files, the first containing the 100 longest sequences, and the second containing the remaining sequences, up to and including the 500th sequence. The 100 longest sequences will be used to create the Clustal alignment and HMMER profile, while the remaining sequences will be used as input to the HMMER alignment and resulting consensus.

4. Create Clustal training alignment

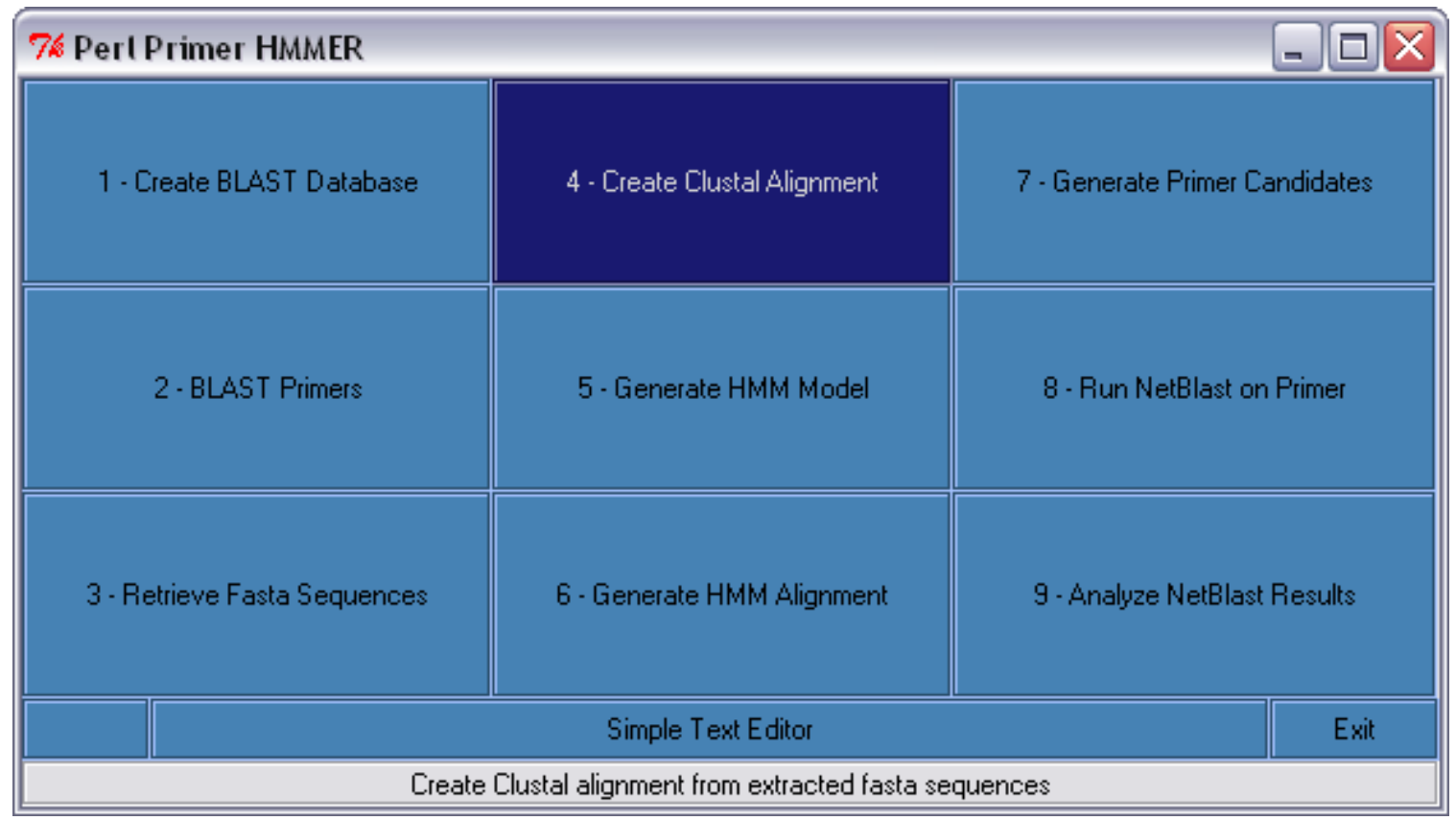

Figure 15 - Create Clustal Training Alignment 


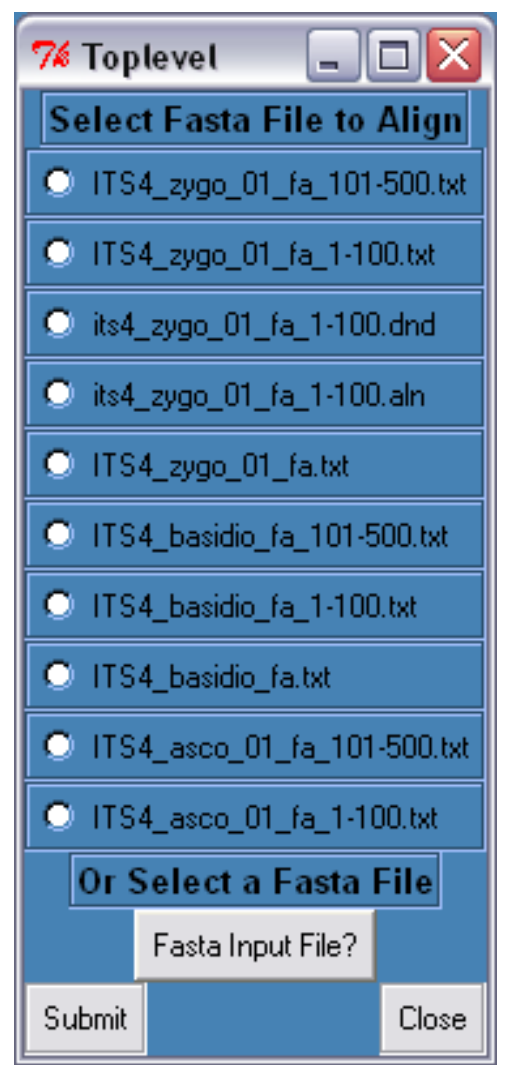

Figure 16 - Selection of Sequences for Alignment

This prompts requests the user to select a fasta file to align. These files are the fasta output files from the previous step. The user also has the option to browse to a specific fasta file, not listed, to create a new Clustal alignment. 
5. GenerateHMMER profile

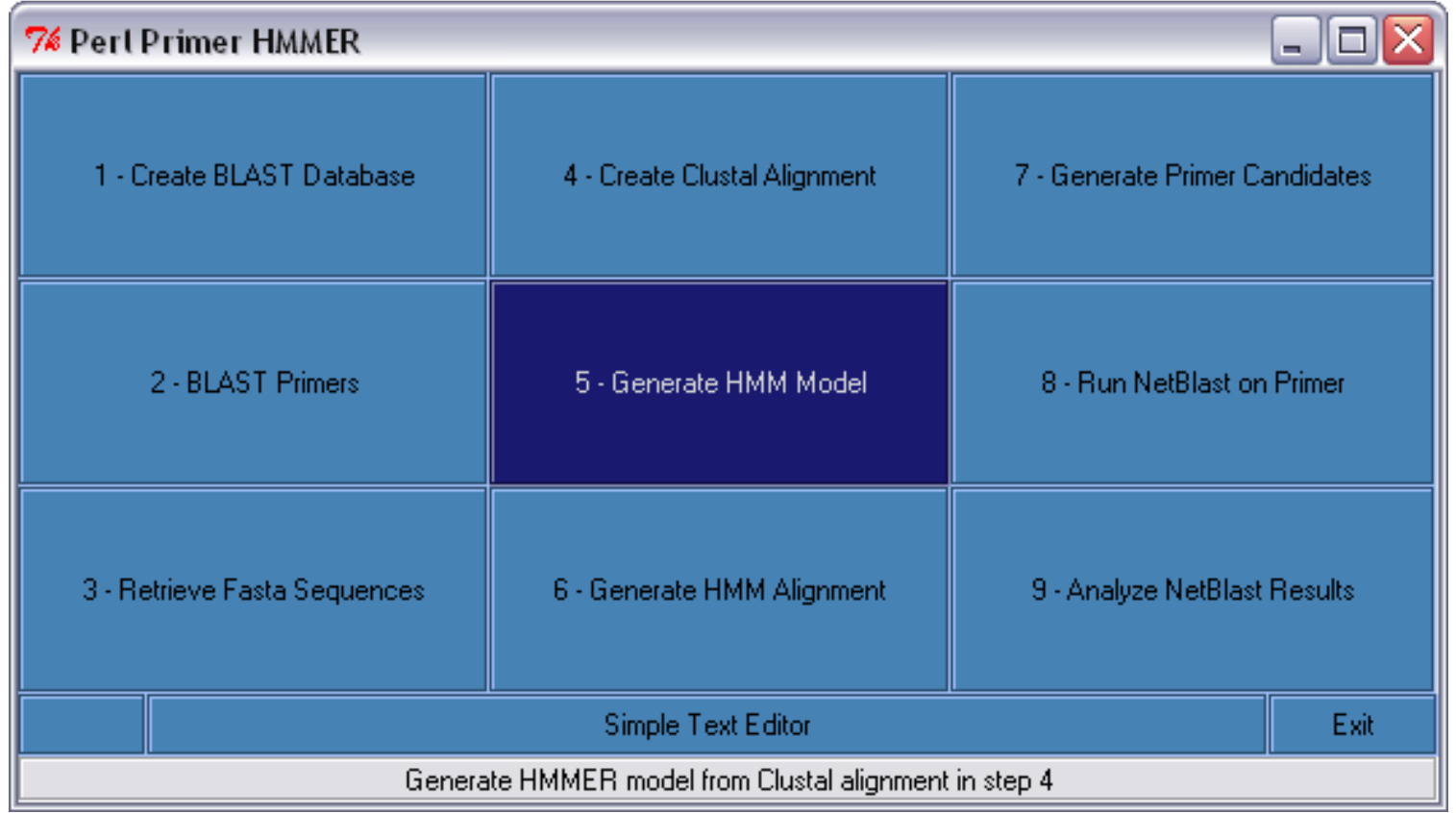

Figure 17- Generate HMMER profile

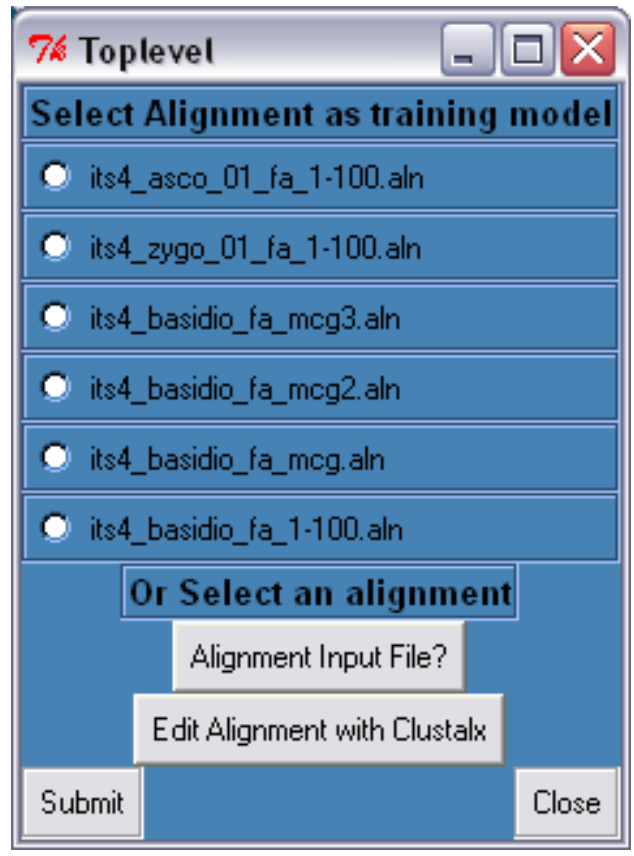

Figure 18 - Selection of ClustalW Alignment as basis for HMMER profile

Several Clustal alignment files are listed for the user. The selected Clustal alignment may be edited or viewed using Clustalx, a graphical implementation of Clustal.

- List commands or reference to commands - how to edit an existing alignment 
- $\quad$ Describe types of editing choices that may be made here

- Sequences extending alignment excessively

- $\quad$ Sequences that differ greatly from overall alignment

- $\quad$ Sequences that are gapped significantly in order to match alignment

- $\quad$ Analyze differences in primer candidates based on edited and non-edited alignment input. This may or may have significant impact on the primer candidates suggested and their accuracy with regard to matching fungal taxa.

Once the user has selected one of the alignment files listed, the file is used as input for hmmbuild, the HMMER tool that will create a training model for HMMER. The model is the statistical representation of the given sequences, with probabilities for changes of state at each point in the sequence.

6.Generate HMMER alignment

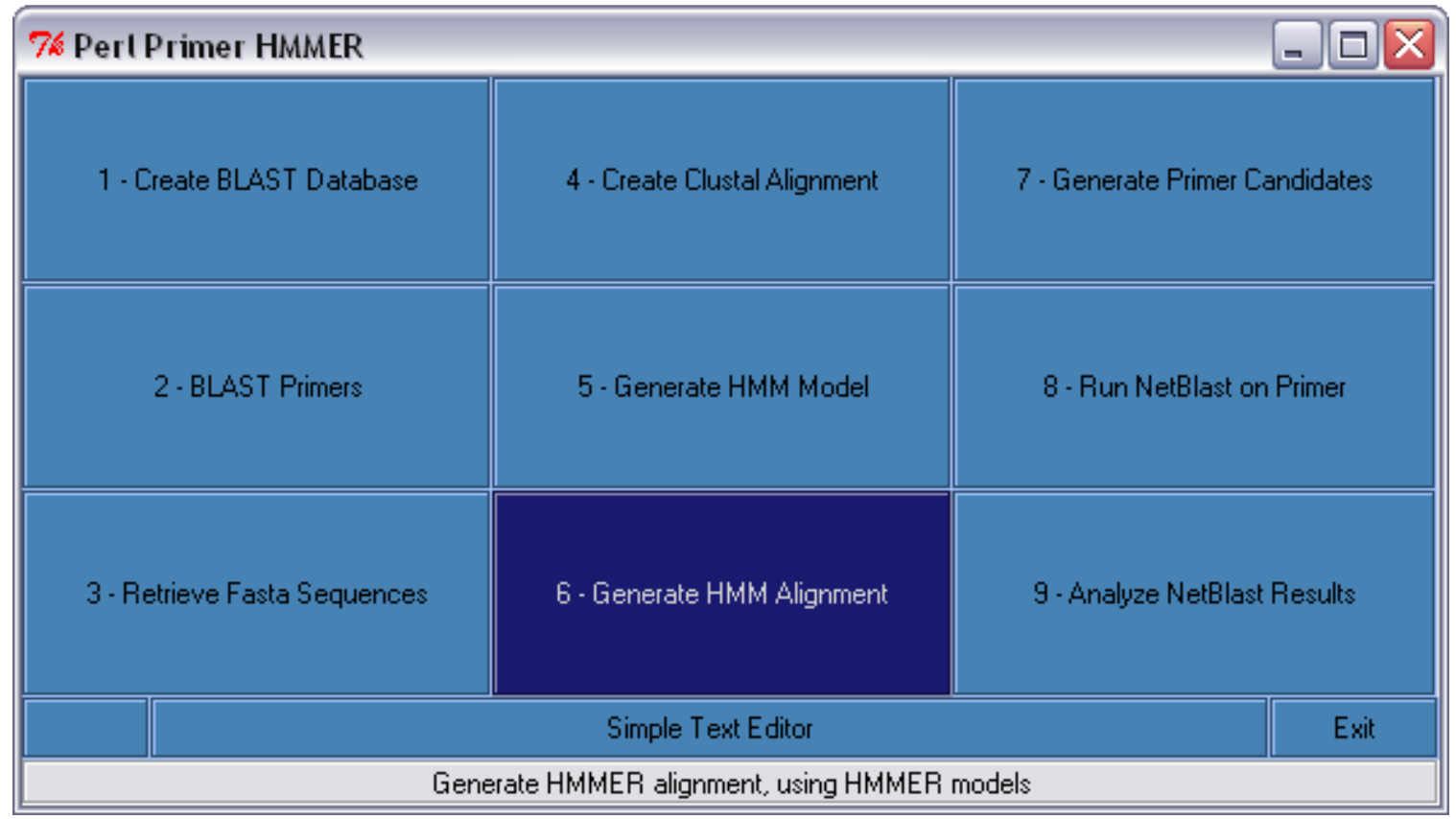

Figure 19 - Generate HMMER Alignment 


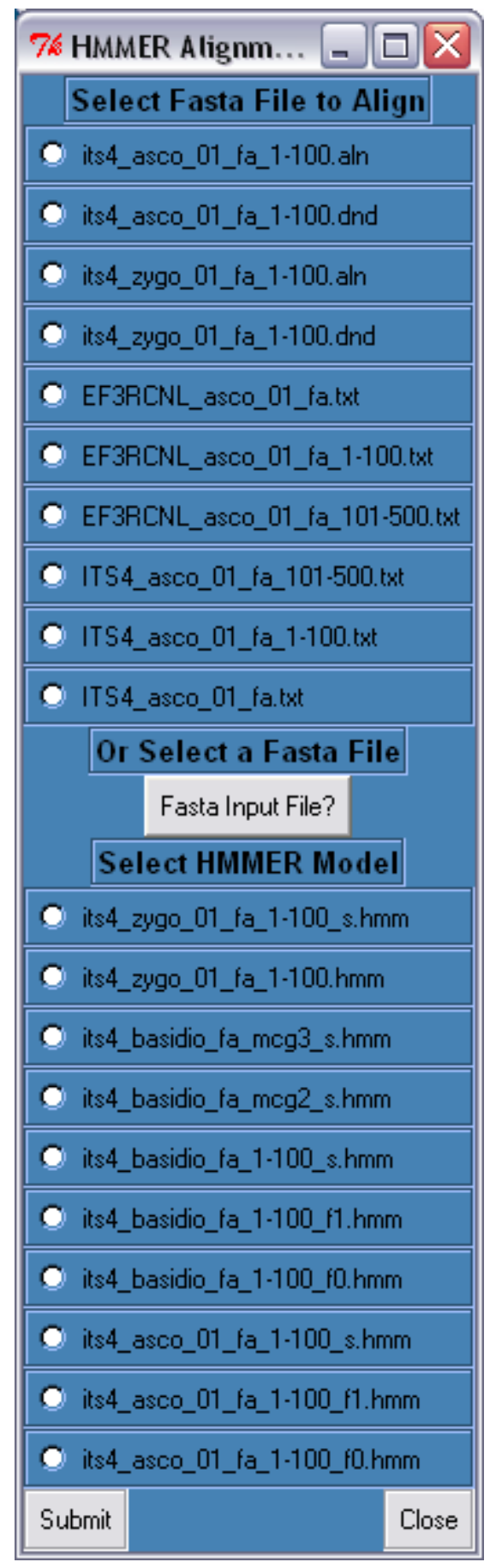

Figure 20 - Selection of Additional Sequences and HMMER profile for HMMER Alignment

Three parameters are used here to create a new HMMER alignment using an existing HMMER profile. First, the output file name for the resulting HMMER alignment, second the input HMMER profile, and finally, the sequences to be aligned, in a FASTA formatted file. 
7.Generate Primer candidates, using chosen technical constraints

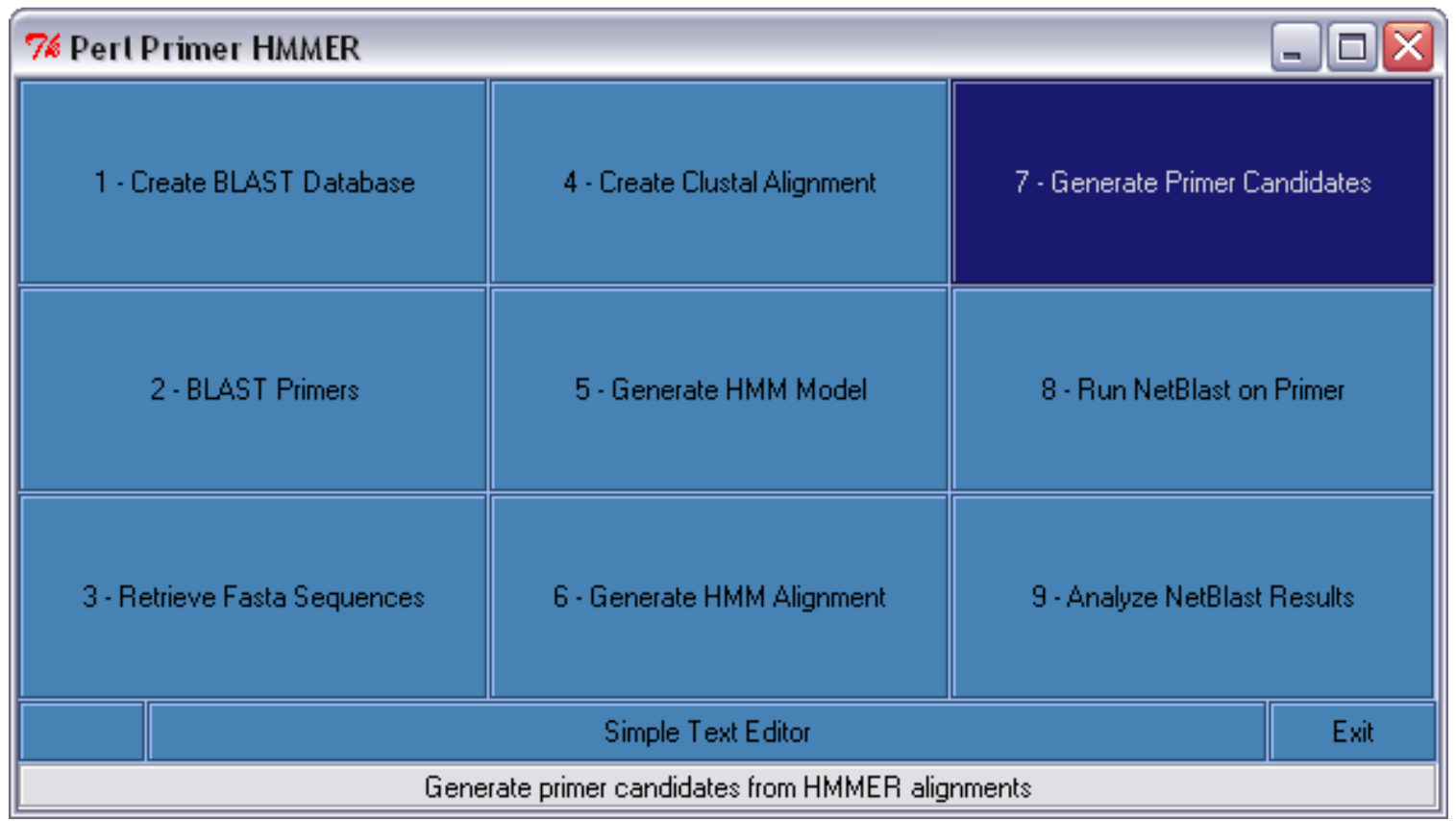

Figure 21 - Generate Primer Candidates

\begin{tabular}{|c|c|c|c|c|c|}
\hline \multicolumn{5}{|l|}{ Open } & $? \times$ \\
\hline \multirow[t]{3}{*}{ Look in: } & \multicolumn{2}{|c|}{$\square$ alignments } & $\nabla$ & \multicolumn{2}{|l|}{ 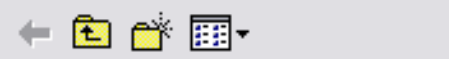 } \\
\hline & \multicolumn{2}{|l|}{ Name } & Size & Type - & Date $\wedge$ \\
\hline & \multicolumn{2}{|c|}{ 國 its4_basidio_fa_mcg3.aln } & $144 \mathrm{~KB}$ & ALN File & $3 / 301$ \\
\hline \multirow{2}{*}{$\begin{array}{l}\text { My Recent } \\
\text { Documents }\end{array}$} & \multicolumn{2}{|c|}{ 國 its4_zygo_01_fa_1-100.aln } & $147 \mathrm{~KB}$ & ALN File & $3 \nmid 11)$ \\
\hline & \multicolumn{2}{|c|}{ 國 its4_asco_01_fa_1-100_fo.hmm } & $13 \mathrm{~KB}$ & HMM File & $3 / 31 \%$ \\
\hline & \multicolumn{2}{|c|}{ 國 its4_asco_01_fa_1-100_f1.hmm } & $115 \mathrm{~KB}$ & HMM File & $3 / 31\rangle$ \\
\hline & \multicolumn{2}{|c|}{ 國 its4_asco_01_fa_1-100_s.hmm } & $49 \mathrm{~KB}$ & HMM File & $3|11\rangle$ \\
\hline & \multicolumn{2}{|c|}{ 國 its4_basidio_fa_1-100_fo.hmm } & $19 \mathrm{~KB}$ & HMM File & $3 / 31 /: \equiv$ \\
\hline & \multicolumn{2}{|c|}{ 國 its4_basidio_fa_1-100_f1.hmm } & $166 \mathrm{~KB}$ & HMM File & $3|31|$ \\
\hline & \multicolumn{2}{|c|}{ 國 its4_basidio_fa_1-100_s.hmm } & $65 \mathrm{~KB}$ & HMM File & $3 / 10 \mid:$ \\
\hline Mu Documents & \multicolumn{2}{|c|}{ 國 its4_basidio_fa_mcg2_s.hmm } & $67 \mathrm{~KB}$ & HMM File & 3/30i: \\
\hline & \multicolumn{2}{|c|}{ 國 its4_basidio_fa_mcg3_s.hmm } & $67 \mathrm{~KB}$ & HMM File & $3 / 30 \mid:$ \\
\hline & 國 its4_zyg & fa_1-100.hmm & $0 \mathrm{~KB}$ & HMM File & $3 / 31 \%$ \\
\hline & 國 its4_zyg & fa_1-100_s.hmm & $48 \mathrm{~KB}$ & HMM File & $3 / 5 / 21$ \\
\hline My Computer & 国:ITS4_as & fa_101-500.ali... & $22 \mathrm{~KB}$ & Microsoft Office Exc... & $3|25|:$ \\
\hline & 国: ITS4_as & fa_101-500_f... & $41 \mathrm{~KB}$ & Microsoft office Exc... & $3 / 31 /:$ \\
\hline & $<1$ & III & & & $>$ \\
\hline My Network & File name: & & & - & Qpen \\
\hline & Files of type: & All Files [ $\left.{ }^{x}{ }^{x}\right]$ & & $\nabla$ & Cancel \\
\hline
\end{tabular}

Figure 22 - Selection of Primer Alignment for Primer Candidate Generation 
This portion of the program calls the external script "Generate_Primer_Candidates.pl," with an HMMER alignment as input, and can also be called on the command line, with an HMMER alignment as a command line argument. Generate_Primer_Candidates.pl constructs a consensus from the alignment, then traverses the consensus, identifying primer candidates. Primer candidates are assessed for GC content, self-hybridizing, selfhybridizing with the candidate reverse, and for melting temperature. Output is in a comma-separated file, with a table listing the potential primers found and their attributes.

8.Run NetBLAST on selected primers

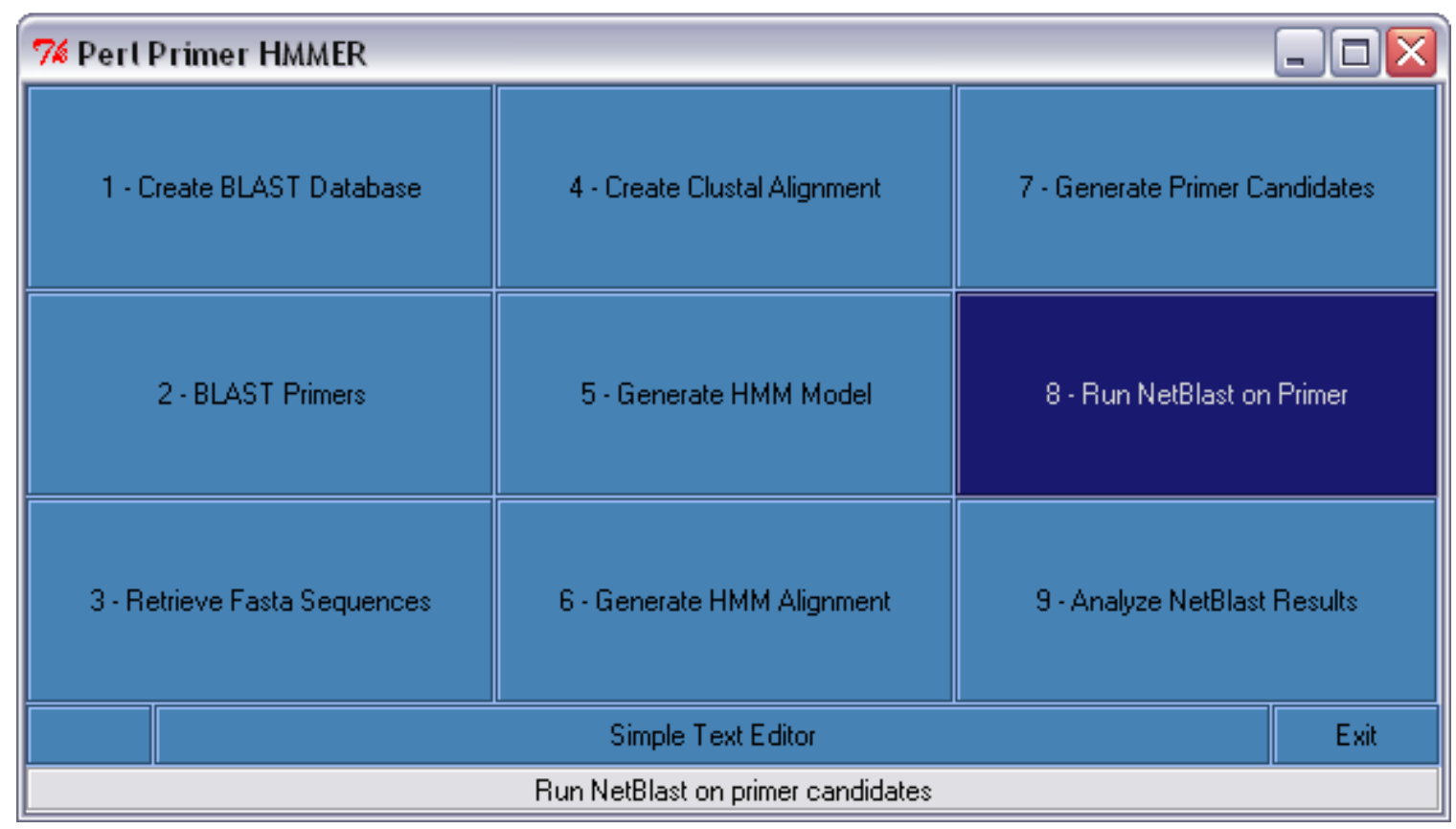

Figure 23 - NetBLAST Select Primers against GenBank 


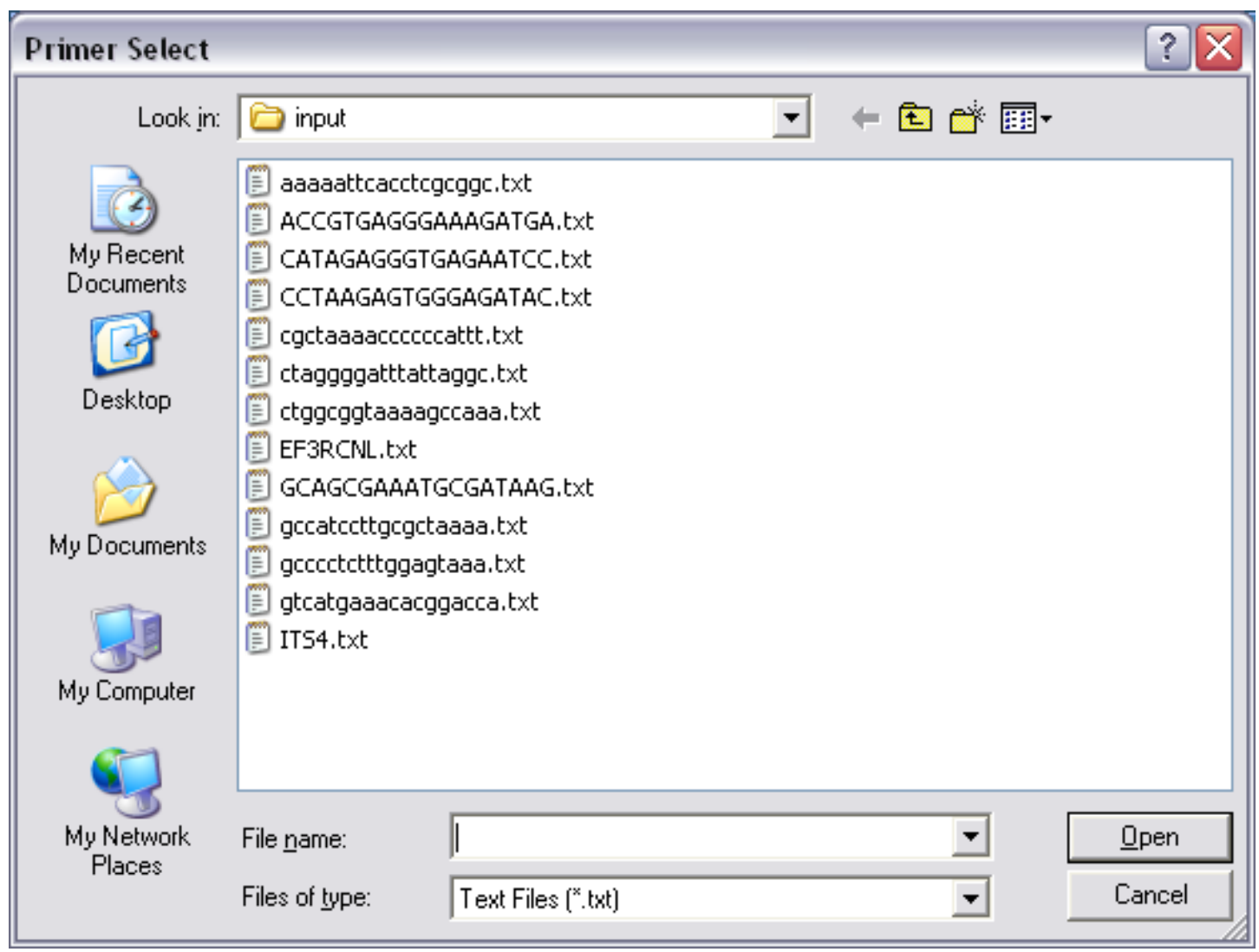

Figure 24 - Primer Selection for GenBank BLAST Processing

NetBLAST (blastcl3.exe) performs a BLAST search of a given primer candidate against GenBank. Input is simply a text file with the given primer string, and output is an html BLAST report. The default number of matches returned is 500, each of which can be accessed by clicking on their respective link in the report. 
9.Analyze NetBLAST result for primer specificity

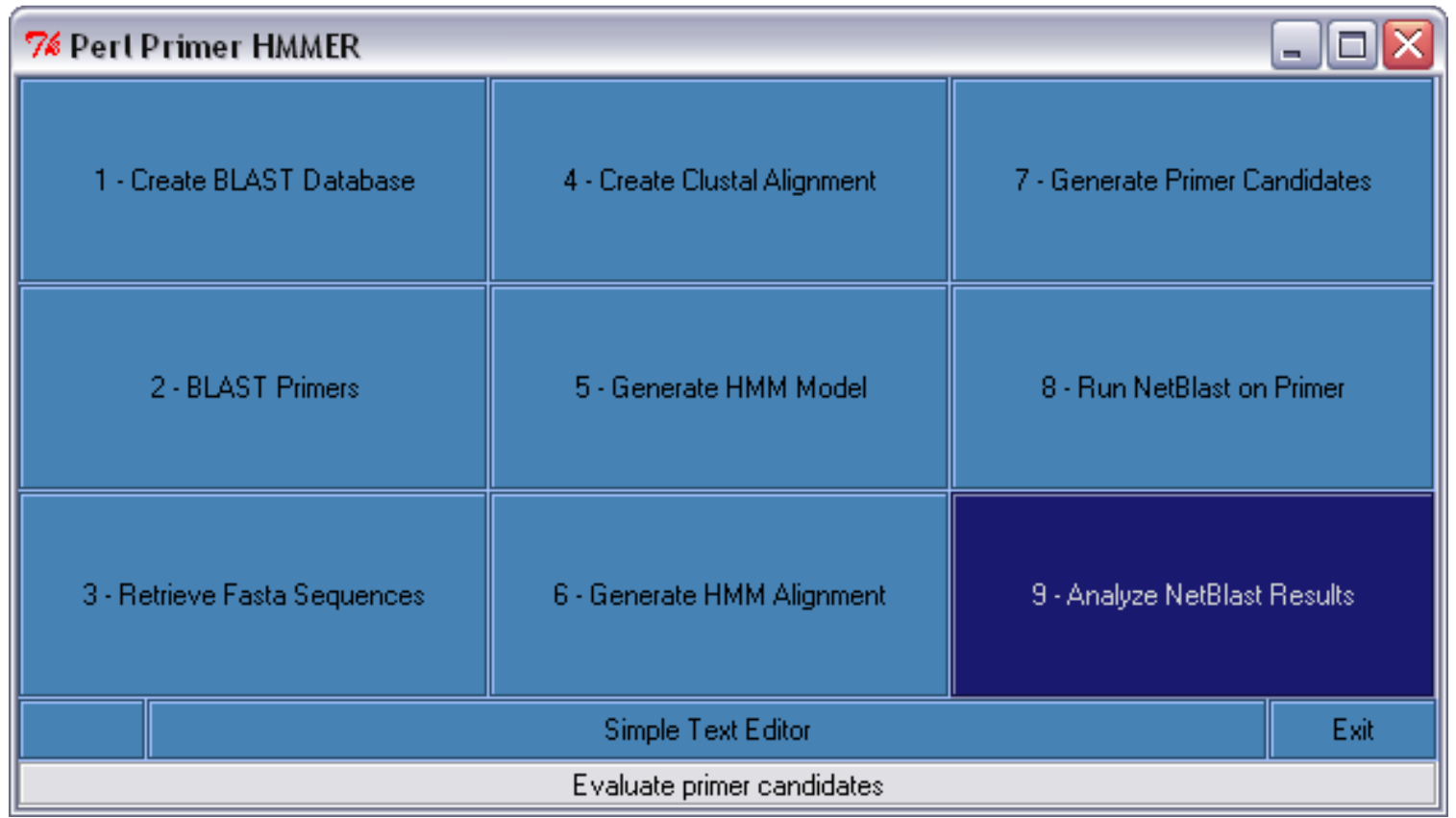

Figure 25 - Evaluate NetBLAST Results

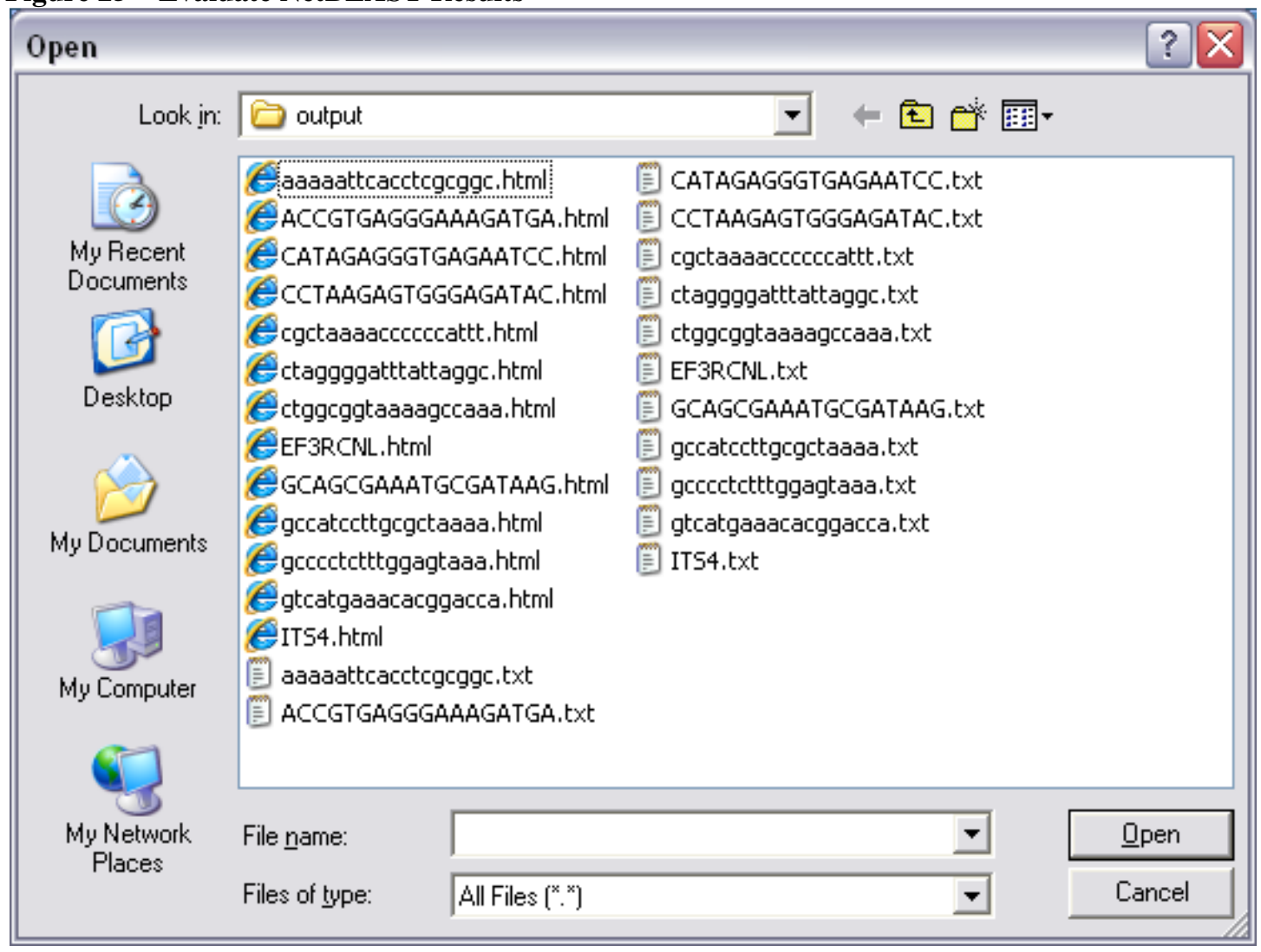

Figure 26 - Select NetBLAST Results for Evaluation

The final portion is an external script Analyze_Netblast.pl, which, like

Generate_Primer_Candidates.pl, can be used with command line input. When called by 
the graphical interface, the user is prompted for a NetBLAST html report. Links for the matching organisms in the report are retrieved and gleaned for taxonomy information. A brief final report is generated, showing counts of matching organisms by taxa. 


\section{Description of Software Harness and Internal Scripts}

The developed software, distinct from the CLUSTAL, BLAST or HMMER software, is comprised of three main scripts or programs: Primer_GUI.pl, Generate_Primer_Candidates.pl and Analyze_Netblast_Results.pl. While

Generate_Primer_Candidates.pl and Analyze_Netblast_Results.pl could be incorporated into the larger Primer_GUI.pl, maintained as a separate script allows the command-line use of the scripts independently from the Graphical User Interface.

\section{Primer_GUI.pI - User interface}

This is the umbrella program, which serves as the primary user interface, tying together multiple software packages. It also provides a suggested workflow for the user, walking them through the primer development process.

\section{Generate_Primer_Candidates.pl - Alignment of Consesnus}

This segment creates a consensus string from the HMMER alignment, which is scanned from possible primer candidates. Once the consensus string has been scanned, a list of primer candidates is produced, which includes scoring for GC content and selfhybridizing, as well as melting temperature.

\section{Analyze_Netblast_Results.pl - Netblast}

The final segment validates a primer candidate against GenBank, cataloging the matching taxa in a way that demonstrates the primer's probable organism specificity. The process parses Netblast result files, and retrieves and scans related GenBank files, retrieved automatically by the program.

The graphical portion of the user interface has been written in Perl Tk. It is relatively simple to update and modify, in part because there is no compiled code. This gives transparency to the interface foundations and the actions executed on behalf of the user. 